\title{
Can a gut helminth parasite influence Th2 inflammatory responses in the skin?
}

\author{
Kimberley Jayne Meijlink
}

A thesis submitted to the Victoria University of Wellington in fulfilment of the requirements for the degree of Master of Biomedical Science

2017 


\section{Acknowledgments}

Firstly, I would like to thank my supervisor Dr. Kara Filbey for all your support, advice, and patience during my Master's project. Your help with teaching me new techniques, assistance during big experiments and all your feedback while I was writing, was very much appreciated. This thesis would not have been possible without your help. Secondly, I would like to thank my supervisor Prof. Graham Le Gros for giving me the opportunity to work on this project, for making sure I had everything I needed to be comfortable here, and for your help and guidance during this project.

Thank you to Dr. Mali Camberis, Dr. Sotaro Ochiai, Angela Jones, and Jodie Chandler for teaching me new lab techniques and for your help during experiments. I would also like to thank the other members of the Allergy and Parasitic Diseases Programme for your advice and help. I am grateful to Karmella Naidoo, Kerry Hilligan, Anna Mooney and all the other members of the PhD club for your assistance and advice.

I would like to thank and acknowledge all the help from the technical staff at the Malaghan Institute. Thanks to the Hugh Green Cytometry Core staff, especially Sally Chappell, and Alfonso Schmidt for your help and support with flow cytometry, and histology. Also thanks to the Biomedical Research Unit staff for taking such good care of all the animals, and teaching me everything I know about working with animals.

This project would not have been possible without project funding from the Health Research Council of New Zealand - thank you very much.

I could not have done this Master's project without the support of my partner, friends and family. Thank you, Eric, for your constant support throughout different time zones. You have taken very good care of me, keeping me fed, sane, and smiling throughout. I will do the same for you when it's your turn again. 
Amanda, you have been my best friend for 20 years, and a constant support my entire life, even from the other side of the world. Thank you for your patience with me and putting up with my absence from Skype.

Mum, Dad, and Grandma, you have always encouraged me, inspired my love of science and discovering new things, and have been a great support. Mike, you have been an amazing brother to me and a constant support this year. Thank you for helping to keep me sane and fed. Your help by cooking dinner after a busy day made a big difference. Auntie Lynne, thank you so much for your support and always being able to make me laugh. You have all been amazing.

This work is dedicated to my grandparents who are no longer with me, but have always encouraged me and have been a moral support throughout my life. 


\section{Abstract}

Helminth parasites are one of the most common infectious agents of humans and cause significant health and economic burdens in the countries they are endemic in, making elimination an important goal. However, epidemiological studies have suggested an inverse correlation between the incidences of infections by helminth parasites in humans and autoimmune and allergic disease prevalence worldwide; it is thought the eradication of parasites in more affluent countries through improved hygiene is an important factor for the increasing incidence of autoimmune and allergic diseases encountered in the Western world.

A Th2 immune response is central in providing immunity against helminth parasites, while suppressing $\mathrm{T}$ helper (Th) 1/Thl7-mediated inflammation and inducing wound repair mechanisms. Helminths have developed strategies to directly regulate the immune response against them to ensure their own survival. Experimental evidence has demonstrated helminths are also able to dampen inflammatory bystander immune responses in their host, via induction of regulatory mechanisms such as regulatory $\mathrm{T}$ cells. These studies have focused primarily on the suppression of food and airway allergies in mouse models and there is limited data on the effect of helminth parasites on skin allergy e.g. atopic dermatitis.

Atopic dermatitis (AD) is a chronic/chronically relapsing Th2 inflammatory skin condition, characterized by skin lesions, dry itchy skin and impaired skin barrier function. This is believed to allow the entrance of other allergens into the body more easily, leading to sensitization and initiation of other allergies later in life, a process termed the 'Allergic March'. With the increased incidence of allergy in the Western world, it is desirable to find new therapies to suppress AD and the onset of the allergic march.

During my Masters, I have investigated whether the gut-dwelling mouse parasite Heligmosomoides polygyrus was able to suppress Th2 responses induced in skin tissue using two different allergy models: 1) intradermal injection (ID) of whole mashed-up house dust mite (HDM), which induces Th2 inflammatory responses, and 2) topical application of the chemical hapten dibutyl phthalate-fluorescein 
isothiocyanate (DBP-FITC), mimicking allergic responses seen in AD. The results show that $H$. polygyrus induces interleukin (IL)-4 production in tissues distal to the gut, including the ear skin tissue, mainly from cluster of differentiation (CD) $4^{+} \mathrm{T}$ cells. Furthermore, helminth infection was able to suppress Th2-mediated inflammation in the skin in both house dust mite and DBP-FITC models, coinciding with an increase in the proportions of regulatory $\mathrm{T}$ cells (Tregs) in skin-associated lymph nodes (LNs). This research further demonstrates the potential use of helminth parasites, or their products, as a therapy for allergic diseases, including those of the skin. 


\section{Table of contents}

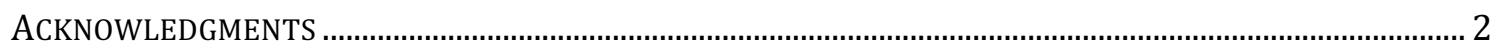

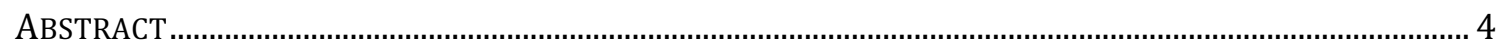

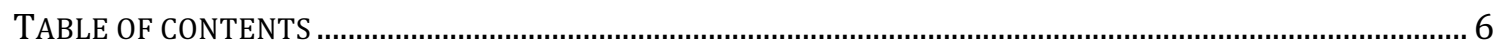

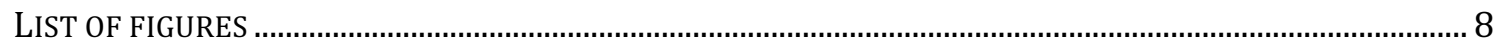

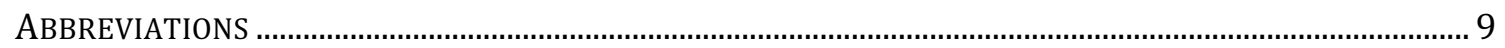

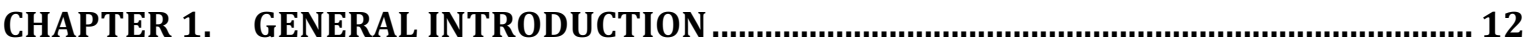

1.1 THE HYGIENE HYPOTHESIS AND HELMINTH PARASITES ..................................................................13

1.1.1 Epidemiological studies of helminth infections and immune disorders...................... 13

1.1.2 Parasitic diseases: symptoms and harmful effects of infection ..................................... 14

1.2 THE TH2 IMMUNE RESPONSE .................................................................................................17

1.2.1 Evolution of the immune system with helminth parasites............................................ 17

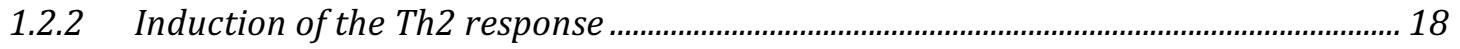

1.2.3 Anti-parasitic mechanisms of the Th2 response............................................................ 19

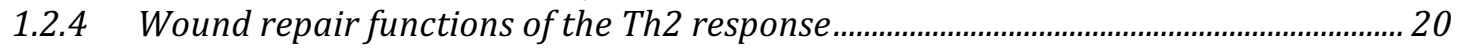

1.3 HELIGMOSOMOIDES POLYGYRUS ……………….................................................................22

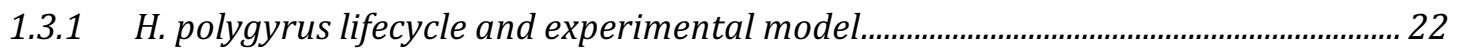

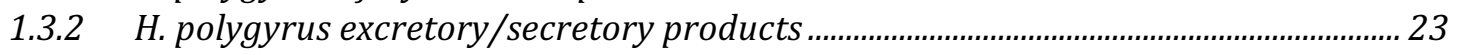

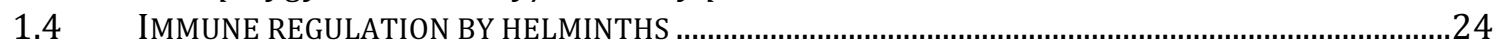

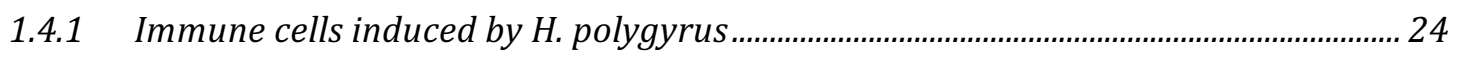

1.4.2 Suppression of the Th2 response by H. polygyrus ........................................................... 26

1.4.3 Three-way relationship between host, parasite and gut microbiota ............................ 28

1.5 ATOPIC DERMATITIS AND THE ALLERGIC MARCH........................................................................29

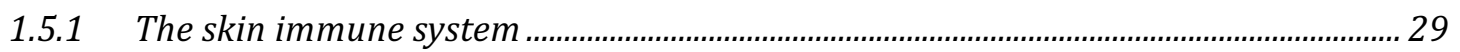

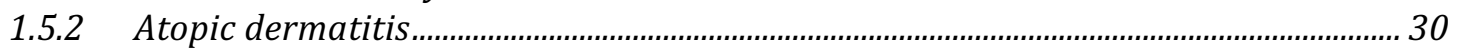

1.5.3 Mouse models of atopic dermatitis...................................................................................... 32

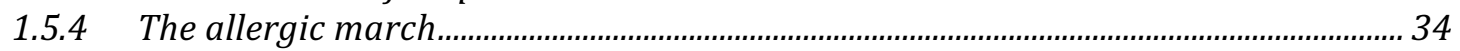

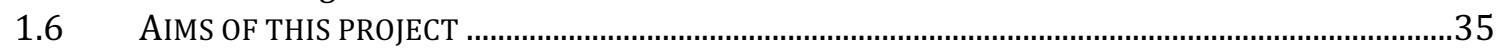

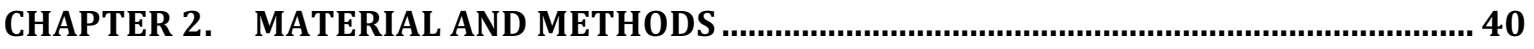

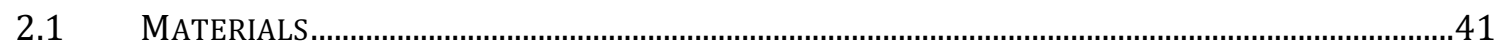

2.1.1 Labware

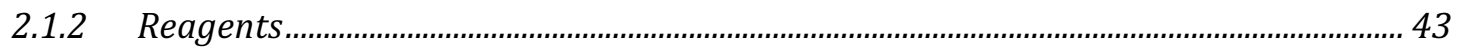

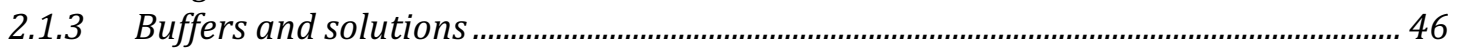

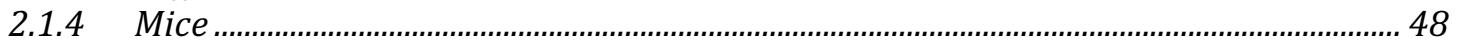

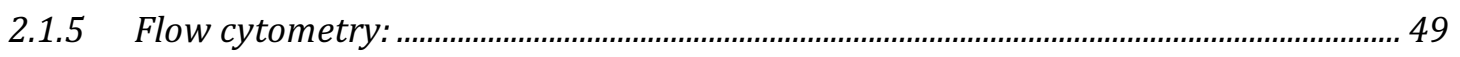

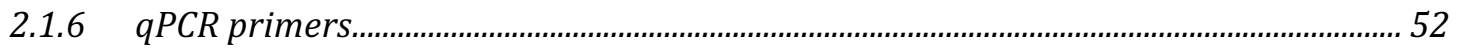

2.2 METHODS.....................................................................................................................

2.2.1 Mouse treatments...................................................................................................................... 53

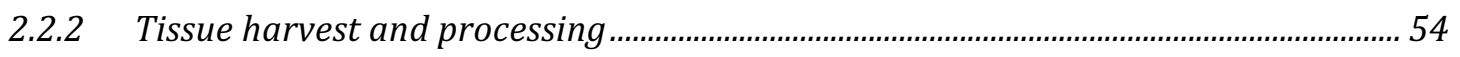

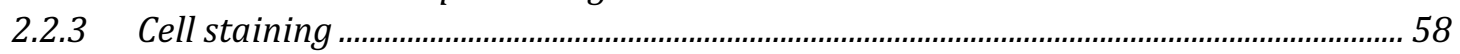

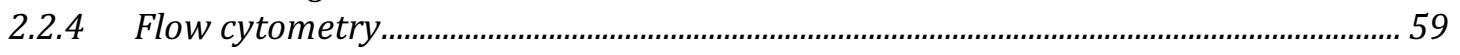

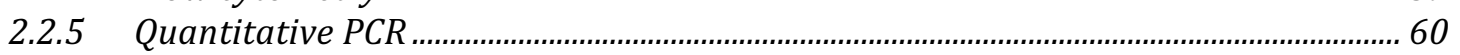

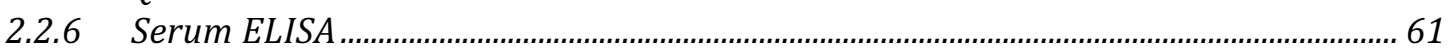

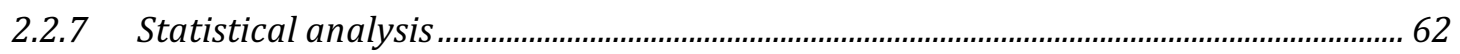

CHAPTER 3. THE EFFECT OF H. POLYGYRUS INFECTION ON SYSTEMIC TH2 RESPONSES IN MICE 63

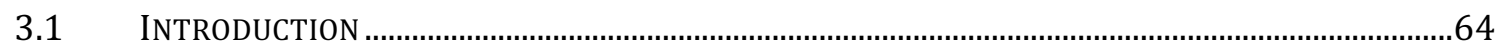

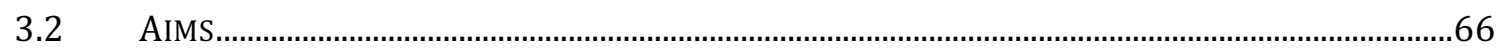

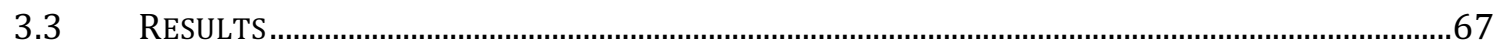

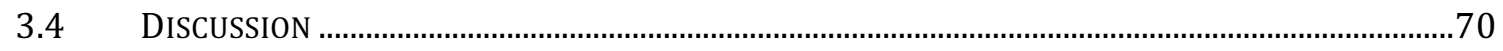

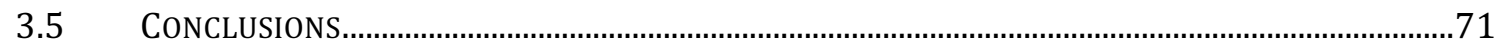


CHAPTER 4. H. POLYGYRUS SUPPRESSES HOUSE DUST MITE-INDUCED TH2

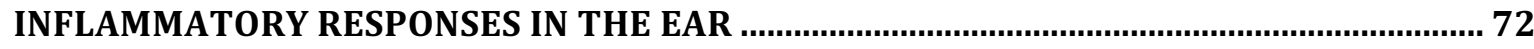

$4.1 \quad$ INTRODUCTION ...................................................................................................................

4.1.1 HDM contains a variety of allergens that induce allergic responses............................ 73

4.1.2 HDM induces Th2 inflammation in the lungs................................................................ 73

4.1.3 H. polygyrus suppresses HDM-induced Th2 inflammation in the lung......................... 74

4.1.4 The use of HDM in skin inflammation models ................................................................ 75

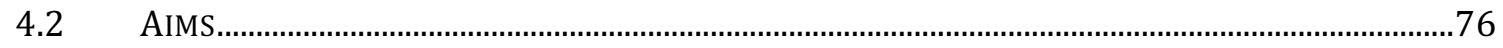

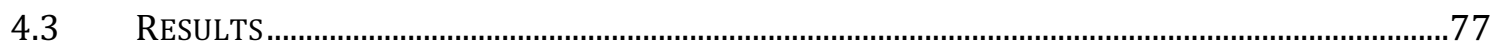

4.3.1 ID injection of HDM in the ear induces substantial Th2 inflammation ....................... 77

4.3.2 H. polygyrus suppresses HDM-induced Th2 inflammation in the skin, coinciding with a slightly increased proportion of Tregs in the $L N$............................................................ 78

4.3.3 H. polygyrus suppresses HDM-induced Th2 inflammation in the skin, coinciding with a slightly increased proportion of Tregs in the ear skin tissue .......................................... 79

4.3.4 Chronic infection with H. polygyrus suppresses HDM-induced Th2 inflammation further 80

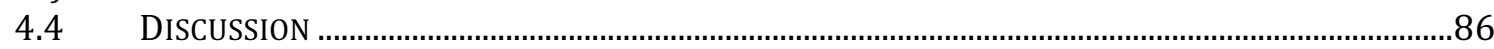

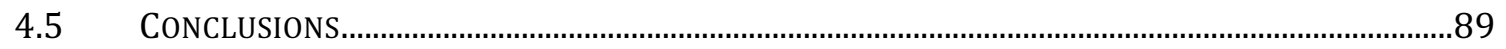

CHAPTER 5. H. POLYGYRUS SUPPRESSES HAPTEN-INDUCED SKIN ALLERGY ............... 90

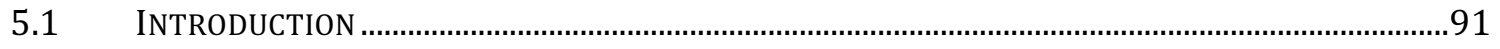

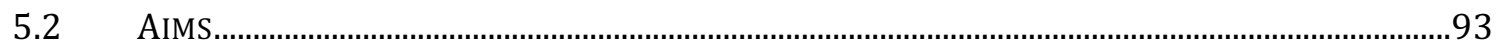

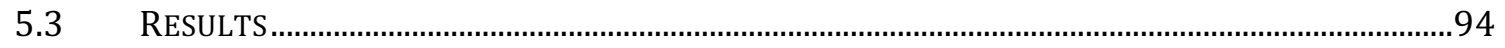

5.3.1 H. polygyrus suppresses skin inflammation in DBP-FITC-induced CHS ..................... 94

5.3.2 H. polygyrus suppresses inflammation early in the immune response but does not

alter DC phenotype in the skin ................................................................................................ 94

5.3.3 H. polygyrus suppresses inflammation and induces IL-4-producing Th2 cells and

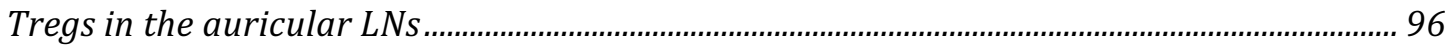

5.3.4 H. polygyrus suppresses Il13 gene expression during peak of inflammation ............ 97

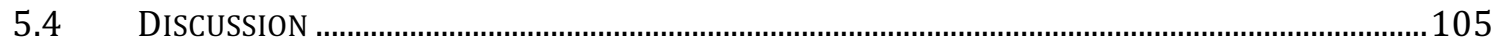

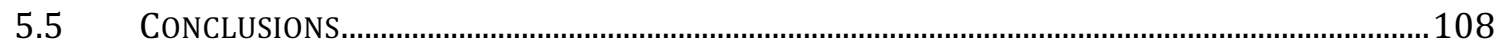

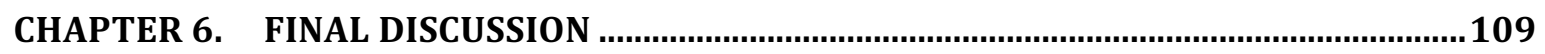

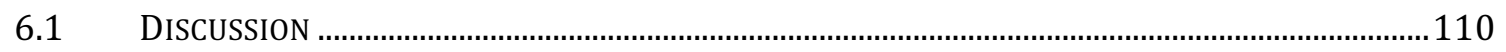

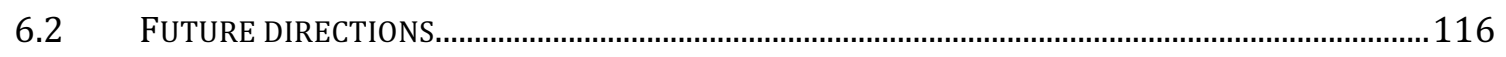

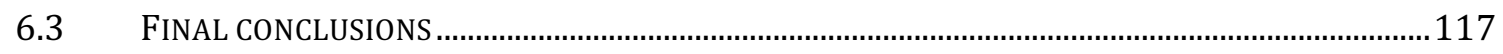

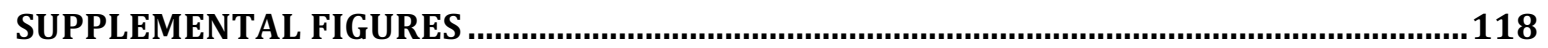

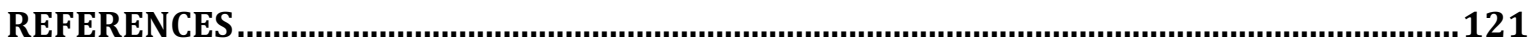




\section{List of figures}

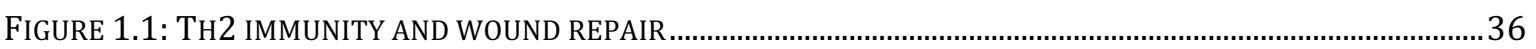

FIGURE 1.2: THE LIFECYCLE OF H. POLYGYRUS IN MICE................................................................................... 37

FIGURE 1.3: IMMUNOMODULATION BY HELMINTH PARASITES …….................................................................... 38

FIGURE 1.4: THE ALLERGIC MARCH ……................................................................................................... 39

FIGURE 3.1: THE EFFECTS OF H. POLYGYRUS INFECTION ON SYSTEMIC TH2 RESPONSES.......................................... 69

FigURE 4.1: TIME COURSE OF ID INJECTED HDM-INDUCED TH2 INFLAMMATION. ............................................... 82

FIGURE 4.2: H. POLYGYRUS SUPPRESSES HDM-INDUCED INFLAMMATION IN THE AURICULAR LN AND INDUCES

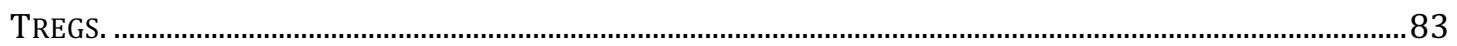

FIGURE 4.3: H. POLYGYRUS AFFECTS HDM-INDUCED INFLAMMATION IN THE EAR SKIN TISSUE AND INDUCES

TREGS AND M2 MACROPHAGES/AAMS..................................................................................................... 84

FIGURE 4.4: H. POLYGYRUS SUPPRESSES HDM-INDUCED INFLAMMATION IN THE AURICULAR LNS DURING

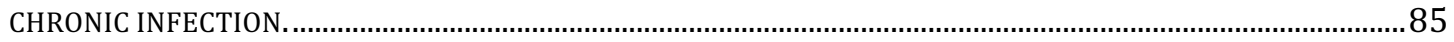

FIGURE 5.1: H. POLYGYRUS SUPPRESSES INFLAMMATION IN A MODEL OF DBP-FITC-INDUCED CHS. ...................99

FIGURE 5.2: MODEL AND GATING STRATEGY FOR ANALYSING DC SUBSETS AFTER TREATMENT WITH DBP-FITC.

100

FIGURE 5.3: $H$. POLYGYRUS SUPPRESSES EARLY INFLAMMATION AFTER DBP-FITC TREATMENT............................ 101

FIGURE 5.4: H. POLYGYRUS SUPPRESSES INFLAMMATION IN THE AURICULAR LNS DURING THE PEAK OF INFLAMMATION AFTER DBP-FITC TREATMENT................................................................................... 102

FIGURE 5.5: H. POLYGYRUS INDUCES TREGS AND IL-4+ T CELLS IN THE AURICULAR LN AFTER DBP-FITC

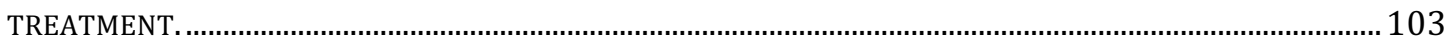

FIGURE 5.6: H. POLYGYRUS SUPPRESSES IL13 DURING THE PEAK OF INFLAMMATION...........................................104

SUPPLEMENTAL FIGURE 1: GATING STRATEGY FOR LN AND EAR TISSUE …………...........................................119

SUPPLEMENTAL FIGURE 2: GATING STRATEGY FOR IL-4 AND IL-13 EXPRESSION IN 4C13R REPORTER MICE.. 120 


\section{Abbreviations}

AAM Alternatively activated macrophage

AD Atopic dermatitis

APC Antigen presenting cell

BALF Bronchoalveolar lavage fluid

BCG Bacille Calmette-Guérin

Breg Regulatory B cell

CAM Classically activated macrophage

CCL Chemokine ligand

CCR7 Chemokine receptor 7

CD Cluster of differentiation

CHS Contact hypersensitivity

CLR C-type lectin receptor

CTLA-4 Cytotoxic T-lymphocyte-associated protein-4

DBP Dibutyl phthalate

DC Dendritic cell

ELISA Enzyme-linked immunosorbent assay

FITC Fluorescein isothiocyanate

FoxP3 Forkhead box protein 3

GWAS Genome-wide association studies

HDM House dust mite

HES H. polygyrus' excretory/secretory products

HIV Human immunodeficiency virus

IBD Inflammatory bowel disease

ICOS Inducible T-cell co-stimulator

Ig Immunoglobulin 


$\begin{array}{ll}\text { IL } & \text { Interleukin } \\ \text { ILC } & \text { Innate lymphoid cell } \\ \text { iTreg } & \text { Induced regulatory T cell } \\ \text { KLRG } & \text { Killer cell lectin-like receptor subfamily G member } \\ \text { LN } & \text { Lymph node } \\ \text { LPS } & \text { Lipopolysaccharides } \\ \text { MFI } & \text { Median fluorescence intensity } \\ \text { MHCII } & \text { Major histocompatibility complex class II } \\ \text { miRNA } & \text { Micro ribonucleic acid } \\ \text { mMCP-1 } & \text { Mucosal mast cell protease-1 } \\ \text { MLN } & \text { Mesenteric lymph node } \\ \text { MMP } & \text { Matrix metalloproteinase } \\ \text { MS } & \text { Multiple sclerosis } \\ \text { NKT } & \text { Natural killer T cells } \\ \text { NLR } & \text { Nucleotide-binding oligomerization domain-like receptors } \\ \text { NOD } & \text { Nonobese diabetic } \\ \text { nTreg } & \text { Natural regulatory T cells } \\ \text { OVA } & \text { Ovalbumin } \\ \text { PAMP } & \text { Pathogen-associated molecular patterns } \\ \text { PAR2 } & \text { Protease activated receptor 2 } \\ \text { PD-L1 } & \text { Programmed death-ligand 1 } \\ \text { PMA } & \text { Phorbol l2-myristate l3-acetate } \\ \text { qPCR } & \text { Quantitative polymerase chain reaction } \\ \text { RAG } & \text { Recombination activating gene } \\ \text { RBC } & \text { Red blood cell } \\ \text { RELM- } \alpha & \text { Resistin-like molecule- } \alpha \\ \text { Standard error of the mean } \\ \end{array}$


STAT6 Signal transducer and activator of transcription 6

T1D Type 1 diabetes

TCR T cell receptor

Tfh $\quad$ T follicular helper cell

TGF $\beta \quad$ Transforming growth factor $\beta$

TEWL Transepidermal water loss

Th T helper (cell)

TLR Toll-like receptor

TN Triple negative

Trl Type 1 regulatory cell

Treg Regulatory T cell

TSLP Thymic stromal lymphopoietin

VEGF Vascular endothelial growth factor

Routes of injection

IP Intraperitoneal

IG Intragastric

ID Intradermal 
Chapter 1. General Introduction 


\subsection{The hygiene hypothesis and helminth parasites}

Parasitic worms (helminths) and humans have co-evolved for many years. ${ }^{1}$ From the $19^{\text {th }}$ century, with increased industrialization, urban living, and improvement of hygiene, these helminths have been eradicated in the Western world. However, allergic disorders such as hay fever have emerged as a "post-industrial revolution epidemic" and over the last 50 years, a steady rise in prevalence has been seen in autoimmune and allergic disorders in Westernized countries. ${ }^{2}$ In 1968, Greenwood et $a l^{3}$ reported a low incidence of rheumatoid arthritis in West Africa, where helminth infection is endemic, and first noted an inverse correlation between incidence of helminth infections in developing countries and immune disorders in more developed countries. In 1989, Strachan found the prevalence of hay fever was increased in people with smaller family sizes and the position they held in a household. ${ }^{4}$ It was hypothesized that declining family size and improvement in hygiene reduced chances of cross-infection in young families, which could result in increased clinical expression of allergic diseases. ${ }^{2,4}$ This theory was named the "hygiene hypothesis".

\subsubsection{Epidemiological studies of helminth infections and immune disorders}

Many epidemiological studies over the intervening years have focused on the hygiene hypothesis. It was found that factors associated with a Western life-style could be responsible for an increased risk of atopy, as with families in higher social economic groups having a higher prevalence of atopic disorders than poorer families in the same country. ${ }^{2,5}$ Furthermore, keeping pets and the sharing of bedrooms is protective, most likely due to the early exposure to allergens, whereas antibiotic use and poor diet (i.e. leading to changes in microbiota) positively correlate with prevalence of atopy in children. ${ }^{6-8}$

In developing countries, significantly lower rates of immune disorders have been recorded, and this is often linked to the endemicity of helminth infections in equatorial and sub-tropical regions. Humans infected with helminths have a lower sensitivity to skin-prick tests and lower levels of allergen specific immunoglobulin 
(Ig)E antibodies. ${ }^{9,} 10$ This protection was reversed when infected people were dewormed with anti-helminth drugs, as demonstrated by an increased sensitivity to allergens during skin-prick tests. ${ }^{11}$ Furthermore, studies have shown that helminths are able to directly induce regulatory mechanisms in humans, demonstrated by an increase in the anti-inflammatory cytokine interleukin (IL)-10 and IgG4 antibody production (discussed further below). ${ }^{9,12}$

Based on their ability to induce immune-regulatory mechanisms in humans, and in numerous mouse studies, ${ }^{9,12-17}$ helminths are being trialled as a new therapy for autoimmune and allergic diseases. So far, the pig whipworm Trichuris suis and hookworm Necator americanus have been used in several trials and proven to be safe and effective in reducing symptoms of immune disorders such as inflammatory bowel disease (IBD), Crohn's disease, asthma and multiple sclerosis (MS). ${ }^{18-21}$

\subsubsection{Parasitic diseases: symptoms and harmful effects of infection}

Helminths infect more than 1 billion people worldwide; ${ }^{22}$ these include schistosomes, hookworms, flatworms, filarial worms, and roundworms. They infect humans via different routes, cause a variety of symptoms and differ in the morbidity they cause.

Hookworms are soil-transmitted helminths and infect over 500 million people in the world. The most common human hookworms are $N$. americanus and Ancylostoma duodenale. ${ }^{23}$ They chronically infect humans, often asymptomatically, indicating adaptation of the parasite to the host's immune system. ${ }^{24}$ They penetrate the skin and migrate through the lungs, ending up in the gut where they burrow through the wall to access the host's blood for feeding. ${ }^{23,25}$ The parasites mature in the gut and secrete eggs into the faeces, which hatch outside the host. The migration and blood feeding of the parasite causes tissue damage, haemorrhaging and anaemia, which can aggravate malnutrition in countries where this is already problematic, leading to growth stunting, impaired cognitive development and diminished physical fitness. ${ }^{25,26}$ 
Schistosomes infect over 240 million people through fresh water and are also able to live within the same host for decades. The main species to infect humans are Schistosoma mansoni, S. haematobium, and S. japonicum. ${ }^{27}$ Infective larvae (cercariae) penetrate through the skin and migrate through the lungs to either the perivascular or mesenteric venules. Mature parasites mate and produce eggs, which can be secreted through faeces or urine, or are trapped in tissues such as the liver and bladder leading to granuloma formation, to wall off the eggs, and fibrosis. ${ }^{27}$ Health complications caused by schistosome infection include: anaemia, growth deficiencies, urinary tract disease, reduced reproductive health, and bowel ulceration. $^{28-30}$

As immunity to most microbes depends on a $\mathrm{T}$ helper (Th) 1/Thl7 mediated immune response, the skewing towards a Th2 response by helminths often has an adverse effect during co-infection with other pathogens. Helminth infections are most common in countries where malaria, tuberculosis and human immunodeficiency virus (HIV) are also prevalent. Studies have shown that helminth parasites could increase susceptibility to tuberculosis and lower efficacy of tuberculosis therapy. ${ }^{31}$ Furthermore, urogenital schistosomiasis, caused by $S$. haematobium, is associated with increased susceptibility to HIV infection. ${ }^{32,33}$ Finally, there is some conflict on the effect of intestinal helminths on the development of malaria as some studies show an increased risk from Plasmodium species in helminth infected individuals, ${ }^{34,35}$ whilst others show helminths protect against Plasmodium infection. ${ }^{36}$

Some vaccines have shown a lowered efficacy in countries where helminths are prevalent, such as the Bacille Calmette-Guérin (BCG) vaccine, likely due to increased IL-10 production in helminth infected people. ${ }^{37}$ Furthermore, the skewing to alternative-activation of macrophages rather than classical activation, could lead to impaired anti-viral and anti-bacterial immunity. ${ }^{38}$ Latent viruses, such as $\gamma^{-}$ herpes virus, can also be reactivated by high levels of IL-4, a cytokine released during the Th2 response elicited by helminths. ${ }^{39}$

While helminth parasites can be protective against some infectious and noncommunicable diseases, it is clear helminth parasites can cause major health issues 
as well. This includes increasing susceptibility to pathogens also prevalent in those countries and altering vaccine efficacy, making the eradication of helminths in developing countries a desirable option. 


\subsection{The Th2 immune response}

The immune system has evolved to deal with many different kinds of pathogens such as viruses, bacteria, parasites and fungi, but also bites, stings and toxins from animals and plants. These pathogens and toxins are neutralized and removed through the initiation of different immune responses in which each component has a different role to play.

Cluster of differentiation (CD) $4^{+} \mathrm{T}$ cells are central to these different responses and can be split into several different subsets based on their cytokine production and transcription factor expression. ${ }^{40}$ While Thl and Thl7 response are important for immunity against micro-organisms such as viruses, bacteria and fungi, they can also cause immune-mediated tissue damage to the host and are involved in autoimmune disorders such as MS and rheumatoid arthritis, via the release of pro-inflammatory cytokines such as IL-12. ${ }^{41-43}$ The Th2 response, on the other hand, is specialized in mediating wound healing after tissue damage and anti-parasitic immunity, but is also involved in mediating allergic diseases (discussed below). ${ }^{44} \mathrm{Th} 2$ cells secrete the canonical cytokines IL-4 and IL-13 to activate innate and adaptive cells involved in the anti-helminth and allergic response. Th9 cells, the main producers of IL-9, were found to be a separate subset of $\mathrm{T}$ cells important for mucus production and activation of mast cells and eosinophils in anti-parasitic immunity and allergic inflammation ${ }^{45-47}$ Regulatory $\mathrm{T}$ cells (Tregs) are known for their secretion of antiinflammatory cytokines (e.g. IL-10 and transforming growth factor $\beta$, TGF $\beta$ ) which prevent chronic inflammation and autoimmune disorders. ${ }^{48,} 49$ These different $\mathrm{T}$ helper subsets are able to balance one another to prevent tissue pathology e.g. the Th2 response can suppress Thl and Thl7 mediated inflammatory responses. ${ }^{42,50}$

\subsubsection{Evolution of the immune system with helminth parasites}

Helminth parasites are multicellular macro-organisms that do not replicate inside the host, but mature and secrete eggs, which hatch outside the host to continue the lifecycle. Some parasites such as hookworms migrate through different tissues which can cause extensive tissue damage and haemorrhaging. Many also feed on 
blood causing further blood-loss and pathology. ${ }^{23,24}$ The Th2 response has most likely evolved to expel or contain these helminth parasites while limiting tissue damage by the immune system (discussed below). ${ }^{44,51}$

Helminths have been shown to induce regulatory mechanisms that suppress inflammation and anti-worm immune responses and also limit tissue damage ensuring not only prolonged worm survival and reproduction, but also the survival of the host (discussed in chapter 1.4). ${ }^{15,24}$

\subsubsection{Induction of the Th2 response}

The first step in the induction of a Th2 response is physical trauma of epithelial barriers and the sensing of danger by epithelial cells such as those found in the gut, lung or skin. Epithelial cells secrete alarmins such as IL-25, IL-33 and thymic stromal lymphopoietin (TSLP), and chemokines, to activate innate cells such as dendritic cells (DCs). ${ }^{52-54}$ TSLP suppresses inflammatory IL-12 production by DCs and activates DCs to adopt a Th2-activating phenotype, which then migrate to the lymph nodes to activate the adaptive arm of the Th2 response. ${ }^{55}$ IL-25 and IL-33 have similar roles in activating innate cells such as macrophages, mast cells, basophils, eosinophils and group 2 innate lymphoid cells (ILC2s). ${ }^{52,54,56-58}$

IL-4 secretion is crucial for the Th2 response and guides the differentiation of naive T cells into Th2 cells. IL-4 signalling, through IL-4R $\alpha$ (a component of the receptors for both IL-4 and IL-13), activates signal transducer and activator of transcription 6 (STAT6) phosphorylation, which upregulates the transcription factor GATA-3 to drive Th2 differentiation. ${ }^{59,60}$ Th2 cells in the lymph nodes secrete IL-4 to induce antibody class switching of B cells to IgE. IgE antibodies are known to activate mast cells and eosinophils in the tissue, causing degranulation and release of proinflammatory molecules, proteins and cytokines. ${ }^{61}$ Th2 cells migrating to the tissue secrete the main cytokines involved in the Th2 response IL-4, IL-5, IL-9 and IL-13. Once in the tissue, positive feedback loops between IL-4/IL-13 on epithelial cells and TSLP/IL-25/IL-33 on Th2 cells may occur to amplify the Th2 response, while IL- 
5 is important for the recruitment of eosinophils and IL-9 can mediate mast cell proliferation. $^{62-64}$

\subsubsection{Anti-parasitic mechanisms of the Th2 response}

After infection with helminths, epithelial barriers are disrupted due to tissue migration or breaching of the gut wall for blood feeding, leading to initiation of a Th2 response (figure 1.1). This section will focus on anti-parasitic mechanisms specific for the gut-dwelling murine parasite Heligmosomoides polygyrus, the helminth utilised in the experiments contained in this thesis, and described in more detail in the subsequent section.

$\mathrm{CD}^{+} \mathrm{T}$ cells are central to immunity against parasites in the gut; treatment with $\alpha-$ CD4 antibodies, blocking IL-4 production, or blocking IL-4R $\alpha$ signalling leads to impaired worm expulsion. ${ }^{65-67}$ Furthermore, the depletion of DCs or blocking of CD80/CD86 resulted in impaired worm expulsion, demonstrating the priming of Th2 cells by DCs is crucial for anti-parasitic immunity. ${ }^{68,69}$

Basophils are activated through receptors including FceRI and ST2 (IL-33R), upon which they secrete cytokines such as IL-4 and TSLP. ${ }^{70}$ They have been shown to migrate to the lymph nodes (LNs) as antigen presenting cells (APCs) and are an important source of IL-4 for the priming of Th2 cells during Nippostronglyus brasiliensis infection. ${ }^{71,72}$ However, depletion of basophils did not have an effect on the priming of Th2 inflammation during $H$. polygyrus infection. ${ }^{73}$

ILC2s are activated by IL-25 and IL-33 and are an important source of Th2 cytokines IL-4, IL-5, IL-9 and IL-13. ${ }^{74}$ IL-4 secretion by ILC2s was recently shown to be important for the priming of Th2 cells in vivo. ${ }^{75}$ IL-13 is believed to induce goblet cell hyperplasia and stimulate mucus production and smooth muscle contraction in the gut, mechanisms crucial to worm expulsion. ${ }^{57}$

IL-5 is important for the maturation and recruitment of eosinophils. Eosinophils release preformed granules through degranulation, containing pro-inflammatory molecules and secrete Th2 cytokines such as IL- $4 .^{76}$ Eosinophilia is a hallmark of 
parasitic and allergic disease, however their exact role in anti-helminth immunity is unclear at present.

Alternatively-activated macrophages (AAMs; or M2 macrophages) are important in protection against many helminth parasites. AAMs are characterized by the expression of gene products such as Yml (an eosinophil chemo-attractant), resistinlike molecule $\alpha$ (RELM- $\alpha$ ), arginase-1, IL-4R $\alpha$ and mannose receptor CD206. ${ }^{24,77}$ AAMs are involved in trapping $H$. polygyrus larvae in the gut wall, which compromises worm vitality and slowly kills them. ${ }^{24}$ AAMs could also harm worms directly through production of arginase-1 resulting in localized deprivation of amino acids. $^{78}$ When AAMs are depleted or arginase blocked, worm expulsion is impaired. $^{78}$

Mast cells proliferate rapidly in parasitic infection and could damage parasites by mucosal mast cell protease-1 (mMCP-1)-mediated breakdown of epithelial tight junction proteins in the host gut, increasing luminal flow and disrupting the niche of the parasites. ${ }^{79,80}$

B cells have varying roles in the protection against different primary helminth infections, and are found to be particularly important for secondary immunity to $H$. polygyrus infection - both IgE and IgGl antibodies, through IL-4 mediated class switching, are induced during infection; ${ }^{81} \mathrm{~B}$ cell deficient mice are unable to clear secondary infections. ${ }^{81}$

\subsubsection{Wound repair functions of the Th2 response}

Some parasites, such as $N$. americanus, migrate through several tissues and cause extensive tissue damage and haemorrhaging in the process. It is believed that the Th2 response has evolved to repair damage done to the tissue at the same time as providing immunity against parasites, as many immune cells and mechanisms are involved in both processes, e.g. mucus production is initiated after disruption of the epithelial barrier, which also functions to expel worms from the gut. ${ }^{44,51,82}$ 
Innate cells are important mediators of wound healing mechanisms (figure 1.1). Eosinophils communicate with other immune cells such as mast cells through the secretion of cytokines such as IL-4 and IL-13. ${ }^{76}$ They also degranulate to release preformed granules containing cytokines, growth factors and matrix metalloproteinases (MMPs) that mediate tissue remodelling, myofibroblast differentiation and wound healing. ${ }^{76,83}$ Mast cells also selectively release cytokines or degranulate after activation, releasing molecules such as vascular endothelial growth factor (VEGF), which is involved in angiogenesis, an important process in wound healing. ${ }^{84}$ AAMs secrete molecules such as VEGF, RELM $\alpha$, argninase-1, Yml, MMPs, TGF $\beta$ and growth factors that are all involved with wound healing mechanisms. ${ }^{85,86}$ Amphiregulin, an epidermal growth factor receptor ligand, secreted by ILC2s is also involved in mediating wound repair, and was seen to help maintain lung homeostasis and repair lung epithelial damage after infection with influenza virus. ${ }^{87}$ Basophils have a less clear role, although they might assist wound repair through secretion of Th2 cytokines such as IL-4. ${ }^{86}$

Innate cells also initiate fibrosis, or scarring, which is defined as over-accumulation of fibrous connective tissue such as collagen in and around damaged or inflamed tissue. ${ }^{86}$ Fibrosis can lead to permanent scarring, malfunctioning of organs and even death. IL-13 was found to induce skin fibrosis in atopic dermatitis (AD) ${ }^{88}$ Arginase-1 secretion by AAMs, however, was seen to suppress fibrosis through the induction of regulatory $\mathrm{T}$ cells. ${ }^{85}$

Taken together, it is clear the Th2 response utilises multiple components to maintain homeostasis by suppressing a tissue-damaging Thl response, clearing parasites, and inducing wound healing mechanisms. ${ }^{44}$ 


\subsection{Heligmosomoides polygyrus}

\subsubsection{H. polygyrus lifecycle and experimental model}

Due to millennia of parasite:host co-evolution, helminths are highly adapted to their specific host, making those adapted to the human immune system difficult to study in the laboratory. ${ }^{89,} 90$ Model helminths that mirror similar lifecycles and migratory pathways are therefore widely used as tools to investigate immune responses against them.

H. polygyrus is a natural mouse parasite, and is thus highly adapted for the murine immune system. It is classified as a nematode parasite and is phylogenetically placed in the same order as human hookworm parasites A. duodenale and $N$. americanus. $^{91}$

In the wild, mice get infected through ingesting the infective larvae, after which the larvae migrate to the small intestine and penetrate through the wall and undergo two developmental moults. At 8 days post-infection, the majority of worms have matured and emerge into the lumen where they coil around the villi of the small intestine, mate and produce eggs, which are released into the host faeces. The eggs hatch outside the host, undergoing two developmental moults to become infective larvae (figure 1.2). ${ }^{79}$

It has been demonstrated that most mouse strains, including C57BL/6 mice, are unable to expel a primary infection with $H$. polygyrus, although a full spectrum of Th2 responses amongst laboratory mice provides fast and slow responders for use in varied experiments. ${ }^{79,92}$ Also, $H$. polygyrus has an entirely enteric lifecycle in the host, making it an excellent model not only to study mechanisms of immunity against and immune modulation by chronic helminth infections, but also to study mucosal immunity and the systemic effects of helminth infections (i.e. changes in immune cell and cytokine profile in tissues distal to the gut). 


\subsubsection{H. polygyrus excretory/secretory products}

As with human helminth infections, $H$. polygyrus has also been found to modify the Th2 response in mice and suppress allergic responses in several experimental settings, mainly through the induction of Tregs (discussed in chapter 1.4). ${ }^{13,16}$ Most likely this modified Th2 response is due to the release of $H$. polygyrus' excretory/secretory products (HES), as it has been demonstrated that HES is able to induce the same protection as a parasitic infection in a murine allergy model. ${ }^{93} \mathrm{HES}$ consists of a mixture of proteins, lipids and carbohydrates, which have been extensively analysed and many have been identified through proteomic studies. ${ }^{94,95}$

More research is needed to find the full array of molecular mechanisms used by helminth parasites to induce immune tolerance, which could lead to novel therapies for common allergic and inflammatory conditions. 


\subsection{Immune regulation by helminths}

\subsubsection{Immune cells induced by $H$. polygyrus}

Regulatory T cells

Tregs have an important role in controlling homeostasis and balancing immune responses during inflammation. These cells are important for the prevention of autoimmune diseases and regulating chronic inflammation, making them an interesting target for drug development. ${ }^{48}$

They are able to suppress $\mathrm{T}$ cell proliferation through cytokine deprivation (such as taking up IL-2 from the environment), and cytolysis. ${ }^{96,97}$ The activation and function of innate cells such as mast cells, basophils, and eosinophils can be inhibited via suppression of IgE antibody production by B cells, thereby preventing activation through Fc $\varepsilon$-receptors. ${ }^{98,99}$ Antigen presentation by DCs can be inhibited by binding of cytotoxic T-lymphocyte-associated protein 4 (CTLA-4) to CD80/CD86, ${ }^{100}$ and the secretion of suppressive cytokines IL-10 and TGF ${ }^{101-104}$

Tregs can either be induced naturally $\left(\mathrm{CD} 4^{+} \mathrm{CD} 25^{+} \mathrm{FoxP} 3^{+}\right.$nTregs $)$in the thymus and are characterised by their specific $\mathrm{T}$ cell receptor (TCR) for self-antigens and express the marker Helios, or induced from effector T cells (iTregs) during the inflammatory response in peripheral tissues (IL-10 producing type 1 regulatory cell (Trl) or TGF $\beta$ producing Th3 cells). ${ }^{99}$ During $H$. polygyrus infection Helios ${ }^{+}$nTregs proliferated early on, but iTregs start to expand one week after infection. ${ }^{105,106}$ This expansion of Tregs was paired with an increase in inhibitory cytokines IL-10 and TGF $\beta$ in the mesenteric lymph nodes (MLNs) from day 14 after infection. ${ }^{16,107}$ Early activation of Tregs was shown to dampen innate and adaptive Th2 responses and increased worm burden, while early depletion of FoxP3 ${ }^{+}$Tregs was seen to increase pathology, T cell activation and Th2 cytokines secretion (IL-4 and IL-13) in the gut. 105, 107

When $H$. polygyrus infection was combined with an airway inflammation model in mice, infected mice had diminished numbers of eosinophils and other immune cells in the bronchoalveolar lavage fluid (BALF) and IL-5 and eotaxin expression was reduced, while IL-10 expression and Tregs were increased. ${ }^{13}$ Using anti-CD25 
antibodies, depletion of Tregs was seen to restore infiltration of immune cells and eosinophils in the BALF. When Tregs from infected mice were transferred to noninfected mice, protection was also transferred. However, protection was also demonstrated when using Tregs from an infected IL-10 deficient mice, suggesting IL-10 does not play a key role in this model of suppressing allergic inflammation in the lung. ${ }^{13}$

\section{Regulatory B cells}

Regulatory B cells (Bregs) are a subset of IL-10-producing B cells thought to be involved in regulating inflammation in autoimmune diseases, and it has been suggested patients with autoimmune diseases have Bregs with an impaired function. ${ }^{108}$ Moreover, Bregs are induced by helminth infections. ${ }^{14,109}$ H. polygyrusinfected mice were found to have a population of $C D 23^{\text {hi }} \mathrm{B}$ cells present in the MLN, that secreted IL-10. Adoptive transfer of these cells from infected mice was sufficient to suppress Der p 1-induced allergic airway inflammation, reducing IL-5 secretion in the BALF, and eosinophil recruitment and immune cell infiltration in the lung. However, IL-10 was not required to provide this protection, suggesting another mechanism or molecules (such as through CD23 and/or TGF $\beta$ ) were involved. ${ }^{14}$

\section{Alternatively-activated macrophages}

AAMs play an important role in the anti-helminth immune response in the gut by trapping helminths in the gut wall and slowly depriving them of nutrients. They are also important for the repair of tissue damage by secretion of many different products such as VEGF, arginase-1, Ym-1 and RELM $\alpha .{ }^{85,86}$ AAMs have also been shown to have an anti-inflammatory role via secretion of anti-inflammatory cytokines IL-10 and TGF $\beta$, suppression of immune cells via expression of programmed death-ligand 1 (PD-L1), and induction of Tregs via retinoic acid dependent mechanisms. ${ }^{110-112}$

$H$. polygyrus infection in mice resulted in the induction of AAMs in various tissues, including the spleen through STAT6-dependent mechanisms. ${ }^{113}$ While these AAMs enhanced bacterial colitis due to suppression of the Thl response needed to kill the bacteria, they were found protective in non-obese diabetic (NOD) mice which develop type 1 diabetes (T1D). ${ }^{113,114}$ In this model, AAMs and Tregs were induced in 
the pancreatic LNs, but this protection was not dependent on the presence of Tregs or IL-10, suggesting a key role for AAMs. ${ }^{114}$

\section{Anti-inflammatory cytokines}

IL-10 is produced by a number of cells including Tregs (Trl cells) and AAMs, and drives differentiation of Trl cells. ${ }^{115,116}$ IL-10 is known for its suppressive effects on immune cells such as APCs and T cells. ${ }^{117}, 118$ IL-10-deficient mice treated with piroxicam develop colitis, and $H$. polygyrus infection inhibited the development of this colitic inflammation. ${ }^{119}$ Furthermore, $H$. polygyrus was protective in a murine model of food allergy, which was dependent on IL-10-mediated blocking of IgE antibody production. ${ }^{120}$

TGF $\beta$ has many roles in the immune response, such as suppressing inflammatory responses, inducing wound healing mechanisms, and restoring tissue homeostasis. ${ }^{44,121} \mathrm{H}$. polygyrus infection induces TGF $\beta$, and it plays an important part in prolonging survival and reproduction of the helminth in the gut as neutralization of TGF $\beta$ in $H$. polygyrus-infected mice results in reduction of worm burden and faecal egg count. ${ }^{122}$ Furthermore, TGF $\beta$ neutralization restored eosinophilic infiltration into the guts and lower levels of IgA antibodies were present in the lamina propria. ${ }^{122}$

One of the proteins found in HES is a TGF $\beta$ mimic, which is highly expressed by adult worms and has been found to induce Tregs in vivo and in vitro through the TGF $\beta$ signalling pathway. ${ }^{15}$ However, the administration of mammalian TGF $\beta$ could not induce the same protection as HES in a mouse allergy model, indicating the TGF $\beta$ mimic is most likely not solely responsible for the suppressive effect of HES. ${ }^{123}$

\subsubsection{Suppression of the Th2 response by $H$. polygyrus}

The wide array of cellular and humoral immune mechanisms induced by helminth parasites has been extensively investigated. ${ }^{77,106,124-126}$ Below is an overview of key immune cells affected by helminth parasites in murine models of infection, with particular reference to the induction and impact of regulatory mechanisms. 
H. polygyrus infection alters the phenotype of the DC populations in the gut. DCs are crucial for the priming of the adaptive arm of the Th2 response against $H$. polygyrus, while innate cells (such as AAMs, eosinophils and ILC2s) are still activated when DCs are depleted. ${ }^{73}$ Several studies showed an increase in the CD11c ${ }^{\text {lo }}$ $\mathrm{CD}_{45 \mathrm{RB}^{\text {mid }}}$ DC population that promoted the induction of Tregs and IL-10 production during chronic infection with $H$. polygyrus, while the CDllchi $D C$ population decreased. ${ }^{127,128}$ This decrease in the CD1lc ${ }^{\text {hi }}$ DC population was thought to be through the blocking of migration of mucosal DCs to the MLN. ${ }^{128}$

ILC2s are rapid responders as they are activated early on in a Th2 response by alarmins, cytokines and inflammatory mediators such as TSLP, IL-25 and IL-33 and when activated, will produce high levels of IL-5, IL-9 and IL-13. ${ }^{129,} 130$ In an ovalbumin (OVA)-Alternaria-induced airway inflammation model, it was shown HES was able to suppress IL-33 secretion, which supressed ILC2 activation resulting in a lower number of ILC2s present in the lung and reduced secretion of cytokines such as IL-5 and IL-13. Furthermore, administration of recombinant IL-33 was shown to negate the suppression of ILC2s by HES. ${ }^{123}$

Another way that helminth parasites influence the immune system is through the release of exosomes. Exosomes are vesicles used by cells to communicate with, and transport proteins and micro RNAs (miRNAs) into, other cells. ${ }^{131}$ Exosomes secreted by $H$. polygyrus are transferred to host cells and have been shown to contain miRNAs that are able to suppress the local innate immune response. Through downregulation of the IL-33 receptor on ILCs, downstream effects on the production of cytokines IL-5 and IL-13 and recruitment of eosinophils was demonstrated. ${ }^{132,133}$

Through suppression of these early responders in the Th2 response, $H$. polygyrus is directly able to suppress not only the adaptive arm of the immune response, but also the innate arm, thereby dampening the whole Th2 response and ensuring prolonged survival and reproduction. 


\subsubsection{Three-way relationship between host, parasite and gut microbiota}

The gut microbiota is essential for the development and homeostasis of the immune system in humans and mice. ${ }^{134} H$. polygyrus is localized in the gut throughout its lifecycle within the host and alters the composition of the gut microbiota, e.g. increasing the abundance of the Lactobacillaceae family, through the secretion of antimicrobial peptides present in HES and through changes in the gut environment. ${ }^{135-137}$ The abundance of Lactobacillus correlated with the presence of total numbers of Tregs in the MLN and transfer of the gut microbiota from infected mice to uninfected mice was enough to induce protection. ${ }^{137,138}$ Together, these results suggest there is a mutual promotion between certain microbes and helminth infections, ensuring both organisms survive within the host while the altering of the microbiota by helminth infections is an important step to indirectly modulate the immune response. ${ }^{136,138,139}$

In conclusion, $H$. polygyrus has been shown to modulate the immune response against it through the induction of a variety of regulatory mechanisms (figure 1.3), which may also act beneficially to dampen concurrent allergic and autoimmune responses. 


\subsection{Atopic dermatitis and the allergic march}

\subsubsection{The skin immune system}

The skin is the first line of defence against pathogens. As such, there are many types of immune cells present in the layers of the skin, both resident and circulating from the skin to draining LNs. They guard against pathogens whilst also maintaining homeostasis to prevent autoimmunity and chronic inflammation by controlling the extent of the immune response. ${ }^{140}$

The skin consists of two main components: the dermis and the epidermis. The epidermis (the outer layer) contains cells such as keratinocytes, melanocytes, Langerhans cells and memory $\mathrm{CD}^{+} \mathrm{T}$ cells (mainly in proximity to the Langerhans cells). ${ }^{140}$ Keratinocytes are crucial for sensing danger signals when the skin barrier is disrupted and pathogens enter the host, as they express several toll-like receptors (TLRs) and inflammasome machinery on their surface. ${ }^{141,142}$ When keratinocytes are activated, they can secrete a variety of pro-inflammatory cytokines, chemokines and antimicrobial peptides to activate an appropriate immune response. ${ }^{140}$

The dermis is more complex in immune cell diversity. The main APC populations are dermal DC populations, plasmacytoid DCs and macrophages. ${ }^{140}$ Dermal DCs can migrate rapidly into the draining LNs to activate T cells, and the $\mathrm{CD} \mathrm{Lb}^{+}$and triple negative ( $\mathrm{TN}, \mathrm{CD}^{2} 26^{-} \mathrm{CD}^{\left.-11 \mathrm{~b}^{-} \mathrm{CD} 103^{-}\right)} \mathrm{DC}$ populations have been shown to induce Th2 responses in a murine model of AD. ${ }^{143}$ Both classically-activated macrophages (CAMs) and AAMs are present in the skin, with CAMs having a broad function in inflammatory responses and AAMs having a regulatory role with the secretion of IL10 and TGF $\beta .^{144}$

There are other innate cells present in the skin, such as basophils, mast cells and ILCs. Basophils are involved in allergic inflammation and immunity against parasites in the skin. They are able to secrete Th2 cytokines such as IL-4 and TSLP, which is believed to be important for the development of AAMs and activation of ILC2s. ${ }^{145}$ Mast cells have granules containing a variety of pro-inflammatory molecules and mediators that can be released upon activation, and can selectively release pro-inflammatory cytokines. Mast cells also have anti-inflammatory 
functions and have been shown to selectively release cytokines such as IL-2 and IL10 inhibiting chronic skin allergy and contact dermatitis in vivo, and they have been shown to directly interact with Tregs in vitro. ${ }^{146-148}$ All three subsets of ILCs (ILCls, ILC2s and ILC3s) are present in the skin. Dermal ILC2s constitutively secrete IL-13 and can interact with mast cells, to suppress their secretion of cytokines. IL-2activated ILC2s were seen to promote inflammation through enhanced secretion of IL-5, eosinophil recruitment and mast cell activation, demonstrating ILC2s can have both pro-inflammatory and regulatory functions in the skin. ${ }^{149}$

Apart from innate cells, there are also diverse populations of $\mathrm{T}$ cells present in the dermis. Skin-resident T cells consisting of innate like T cells (such as $\gamma \delta \mathrm{T}$ cells and natural killer $\mathrm{T}(\mathrm{NKT})$ cells), and memory $\mathrm{CD} 4^{+}$and $\mathrm{CD}^{+} \mathrm{T}$ cells have been shown to be present in low numbers. ${ }^{150}$ These are thought to be major players in homeostasis, wound healing and in the inflammatory response where they are the first line of defence against pathogens in the skin. ${ }^{151}$ Adaptive $\mathrm{CD} 4^{+} \mathrm{T}$ cells that are recruited to the skin by DCs are mainly Thl, Th2 or Th17 cells. ${ }^{140}$

\subsubsection{Atopic dermatitis}

Skin disorders are associated with the dysregulation of the immune system in the skin. AD, or atopic eczema, is a chronic, relapsing Th2 inflammatory skin condition characterized by pruritus (itching) and inflammatory skin lesions. ${ }^{152,153}$ Over the past 30 years, prevalence of this disease has been increasing in developed countries and now seems to have plateaued at $10-20 \%$ of the population. ${ }^{154}$ In more than half of the cases (around 60\%), onset of this disease is within the first year of life, and most of the time it greatly improves or resolves later in childhood. ${ }^{152}$ However, the disease can appear at any age, and when it appears to be outgrown, patients will still have sensitive hyper-reactive skin and might even have recurrences. ${ }^{152}$ Furthermore, studies have shown onset of early AD can often lead to increased susceptibility to food allergies, asthma or other airway allergies later in life, a phenomenon called the atopic or allergic march (figure 1.4, discussed below). 
The cause of disease is not fully understood, however it seems to be multifactorial through interactions between environmental and genetic factors. The highest risk factor seems to be family history of $\mathrm{AD}$, of which the most well characterised genetic factors are null mutations in the Filaggrin gene, an important structural molecule involved in barrier function and immune mechanisms such as innate cell signalling and $\mathrm{T}$ cell activation. ${ }^{155,156}$ Well-known environmental factors include a Western diet (high sugar and fat, low fibre diet), small households, and an urban lifestyle. ${ }^{152,153}$

The main mechanisms of $\mathrm{AD}$ are abnormal functioning and structure of the epidermis (such as disrupted skin barrier function, increased abundance of Staphylococcus aureus and increased water loss) and an inappropriate immune response to antigens in the skin causing allergic inflammation. ${ }^{152}$

Experiments using in vivo models have started to unravel important mechanisms in the onset and persistence of AD. Barrier disruption is an important factor in the induction of skin inflammation with alarmins such as TSLP, IL-33 and IL-25 secreted by keratinocytes after disruption of the skin barrier. ${ }^{157}$ These alarmins activate innate cells such as ILC2s, DCs, mast cells and basophils. ${ }^{52,53}$ DCs, after activation, migrate to the skin draining LNs, activating the adaptive arm of the immune response. Of the adaptive arm, Th2 cells and IgE-producing B cells are mainly associated with a Th2 response. Th2 cells migrate to the skin and secrete IL4, IL-5, IL-9 and IL-13, while Th2 cells in the LN mainly secrete IL-4. ${ }^{157}$

Mast cells are primarily activated through FceRIII by binding of IgE, but can also be directly activated by alarmins such as IL-33. ${ }^{158}$ Mast cells are located close to nerve endings, and have been suggested to have a role in the neurogenic components in the itching response of $\mathrm{AD}$ through the tryptase/protease-activated receptor 2 (PAR2) pathway. ${ }^{153}$ ILC2s accumulate in AD skin lesions, and are the main source of IL-13. ${ }^{159}$. Basophils also accumulate in AD skin lesions, where they are a main source of IL-4 and eotaxin, promoting infiltration of eosinophils. ${ }^{145}$

Tregs have also been shown to be important in regulating inflammation in atopy such as AD. ${ }^{99,160}$ It was shown depletion of forkhead box protein 3 (FoxP3) ${ }^{+}$Tregs in 
an in vivo model increased skin inflammation through DC and T cell activation and proliferation, and increased IgE serum levels. ${ }^{160}$ Furthermore, they found that inducing airway allergy after sensitization and challenge through the skin route, Treg depletion increased lung immune cell infiltration, and higher infiltration of $\mathrm{CD}^{+} \mathrm{T}$ cells and eosinophils in the BALF. ${ }^{160}$ These results indicate Tregs are important in regulating allergic responses in the skin and preventing development of subsequent airway allergy.

\subsubsection{Mouse models of atopic dermatitis}

Murine models for $\mathrm{AD}$ can be divided into three categories: epicutaneous application, transgenic mice, and mice spontaneously developing AD-like skin lesions. $^{161}$

\section{Epicutaneous application of haptens}

Many chemicals have been used as a topical treatment, mimicking the constant exposure to external factors, to effectively induce AD in mice. Two examples are described below:

Fluorescein isothiocyanate (FITC) has been shown to induce contact hypersensitivity (CHS), which is a common form of AD, mostly mediated by Th2 cells. Dibutyl phthalate (DBP) is used as a solvent, and it has been shown to enhance the potency of allergens such as FITC. ${ }^{162}$ Epicutaneous application of DBPFITC was shown to induce TSLP production, activating DCs and inducing Th2 inflammation and AD-like skin lesions. ${ }^{143}$ As various chemicals are able to induce CHS upon repeated exposure to the skin in humans, topical application of DBPFITC has proved to be an effective model to study AD in mice. ${ }^{162}$

MC903 (calcipotriul; Dovonex) is a low-calcemic analogue of vitamin D3 (1 $\alpha, 25-$ $(\mathrm{OH}) 2 \mathrm{D} 3)$ and used for the treatment of psoriasis in humans. ${ }^{163}$ MC903 has been seen to effectively induce AD like symptoms and is used to study AD in mice. It is dissolved in ethanol and applied topically on the ears of mice and induces AD-like symptoms such as increased skin thickness, dry scaly skin and scratching behaviour suggesting pruritus. ${ }^{163}$ MC903 induces TSLP production by keratinocytes, and an 
increase in Th2 inflammation was seen in MC903-treated ears. ${ }^{163,} 164$ This demonstrates MC903 induces a skin condition mimicking human AD.

\section{Transgenic mice}

Transgenic mice are used to study the importance of specific components of the immune system on the development and progression of $\mathrm{AD}$, such as cytokines, chemokines and specific genes found in genome-wide association studies (GWAS). ${ }^{161,165}$

Transgenic mice overexpressing IL-4 spontaneously develop pruritus at 4 months and have impaired wound healing with enhanced angiogenesis and Th2 inflammation, symptoms closely resembling $\mathrm{AD} .^{161,166}$

Transgenic mice overexpressing TSLP develop Th2 inflammation closely resembling $\mathrm{AD}$. When these mice were crossed with mice lacking T cells (TCR $\beta^{-/-}$mice), AD still developed, demonstrating the onset of disease is not dependent on T cells. ${ }^{161,167}$

Flaky tail $\left(\mathrm{FLG}^{\mathrm{ft}}\right)$ mice have reduced expression of filaggrin, which has shown to result in dry, flaky skin which resembles skin lesions in $\mathrm{AD}$, and enhanced susceptibility to sensitization with epicutaneous antigens. ${ }^{168}$ As loss-of-function mutations in filaggrin are seen in up to $50 \%$ in patients with $\mathrm{AD}$, it is shown to be an important factor in the development of the disease, although not solely responsible. $^{169}$

\section{Spontaneous development}

$\mathrm{Nc}$ /Nga mice develop spontaneous AD-like lesions under conventional conditions, but not under specific-pathogen free (SPF) conditions, demonstrating an additional environmental factor involvement (the microbiota). Scratching behaviour, erythema and haemorrhaging is seen after 8 weeks and is followed by development of dry and scaly skin, oedema, and retarded growth. ${ }^{170}$ The skin lesions are characterised by infiltration of $\mathrm{CD} 4^{+} \mathrm{T}$ cells, eosinophils, mast cells, high levels of Th2 cytokines, and the mice have elevated serum levels of IgE. ${ }^{153,161,171}$ The frequency of developing skin lesions in mice kept under SPF conditions varies greatly between laboratories, therefore in some groups $\mathrm{Nc} / \mathrm{Nga}$ mice also receive some form of epicutaneous treatment to induce AD. ${ }^{153}$ 


\subsubsection{The allergic march}

The allergic march is described as the progression from AD in early childhood to other allergies such as food allergy, asthma and allergic rhinitis later in childhood, although this can occur at any age. ${ }^{172}$ Evidence has supported this relationship between allergic diseases, and it has been estimated around one in three patients with $\mathrm{AD}$ develop asthma later on in life, and two in three patients develop allergic rhinitis. ${ }^{172,173}$ One mechanism through which the allergic march may occur is through the defective skin barrier. Due to the activated state of immune cells in the skin, further sensitization to other allergens may occur more readily in AD patients, thus making them more susceptible to increased allergic responses in early life. (figure 1.4). ${ }^{173}$

It has been demonstrated experimentally that sensitization through the skin can indeed result in allergic responses in distal tissues. Where $\mathrm{AD}$-like responses were defective in mice lacking TSLP specifically in the skin, airway inflammation after intradermal (ID) sensitization and intranasal challenge was also attenuated. ${ }^{174}$ These results not only demonstrate the importance of TSLP in the development of allergic responses, but also the importance of the sensitization stage in the skin for developing allergic airway inflammation. Double mutant flaky tail $\left(\mathrm{Flg}^{\mathrm{ft} / \mathrm{ft}}\right)$ mice that develop spontaneous $\mathrm{AD}$, developed spontaneous lung inflammation later in life. ${ }^{175}$ Ragl $^{-/-}$Flg $^{\mathrm{ft} / \mathrm{ft}}$ mice still developed AD (although skin pathology was less severe) in the absence of $\mathrm{B}$ and $\mathrm{T}$ cells, but did not develop lung inflammation, demonstrating the adaptive arm of the Th2 response could be crucial for the initiation of the allergic march. ${ }^{175}$

It seems disrupted skin barrier and sensitization in the skin is crucial for the initiation of the allergic march. Therefore, preventing the onset of AD would be highly beneficial for the health of patients. 


\subsection{Aims of this project}

H. polygyrus effectively suppresses allergic inflammation in the guts and lungs through the induction of regulatory mechanisms such as Tregs. ${ }^{13,15,16}$ Tregs have also been shown to be important for regulating inflammation in the skin during AD, and Treg depletion led to increased inflammation through DC activation of Th2 cells. ${ }^{160}$ This suggests Tregs induced by $H$. polygyrus might also be able to suppress allergic responses in the skin. However, there is a limited number of publications addressing the effect of helminth parasites during skin allergy in both epidemiological studies and murine models, and there is a need for further investigation in this area. ${ }^{176,177}$ Furthermore, suppression of Th2 inflammation in AD lesions by helminth parasites may also prevent initiation of the allergic march, and therefore, prevent the onset of other allergic diseases later in life.

Therefore, the overall aim of this project is to study if the gut dwelling mouse parasite $H$. polygyrus is able to suppress Th2 inflammatory responses induced in skin tissue.

Specific goals:

1) To determine if $H$. polygyrus alters immune cell and cytokine profiles in the skin tissue, via use of 4Cl3R (IL-4 and IL-13) dual reporter mice to study expression profiles of the canonical Th 2 cytokines IL- 4 and IL-13 by immune cells in various tissues by flow cytometry.

2) To assess if $H$. polygyrus is able to suppress Th2-mediated inflammation induced by two different allergy models, i.e. treatment via an ID injection of house dust mite (HDM) or topical application of the chemical hapten DBPFITC in the ear skin tissue.

3) To identify if $\mathrm{H}$. polygyrus induces $\mathrm{CD}^{+} \mathrm{FoxP}^{+}$Tregs in the auricular LNs and ear skin tissue.

4) To characterize differences in the Th2 inflammatory response following treatment of $H$. polygyrus-infected mice in comparison to uninfected mice. 


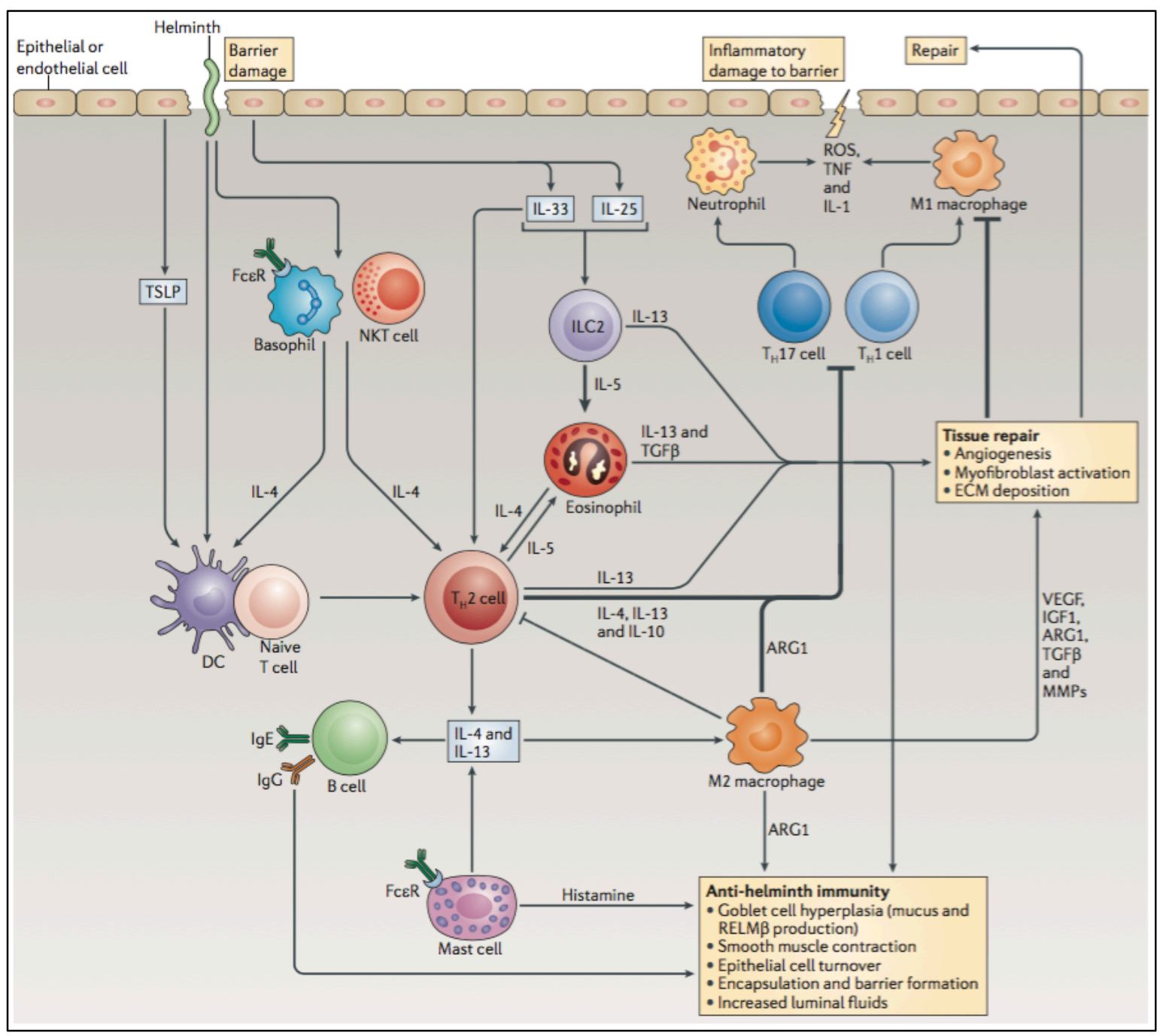

\section{Figure 1.1: Th2 immunity and wound repair}

When helminth parasites infect the host, epithelial barriers such as those in the skin, lungs or gut are disrupted. In the skin, damage initiates secretion of alarmins by keratinocytes such as TSLP, IL-25, and IL-33 that induce a Th2 response. This response acts to simultaneously repair tissue damage and mediate killing and expulsion of the worm. ILC2s, activated by IL-25 and IL-33, are the main source of IL-5, which activates eosinophils, and IL-13, which plays a role in both helminth expulsion from the host (e.g. goblet cell hyperplasia, smooth muscle contraction, epithelial cell turnover) and tissue repair (e.g. angiogenesis, myofibroblast activation and extracellular matrix (ECM) deposition). TSLP activates DCs, which migrate to the draining LNs to activate $\mathrm{T}$ cells and drive Th2 differentiation. Basophils are also thought to be an important source of IL- 4 for Th2 differentiation. Th2 cells produce IL-5, IL-13, and IL-4, which can induce B cell class-switching to IgE and IgGl, and induce M2 macrophages. Activated B cells produce parasite-specific antibodies which activate basophils and mast cells. Mast cells and M2 macrophages secrete effector molecules to mediate parasite killing and wound repair. Tissue repair is also induced by suppressing tissue damaging Thl- and Thl7-mediated immune responses through production of IL-4, IL-10 and IL-13 from Th2 cells and Argl from M2 macrophages. (Gause, W.C., Wynn, T.A. \& Allen, J.E. Type 2 immunity and wound healing: Evolutionary refinement of adaptive immunity by helminths. Nat Rev Immunol 13, 607-614 (2013)). ${ }^{44}$ 


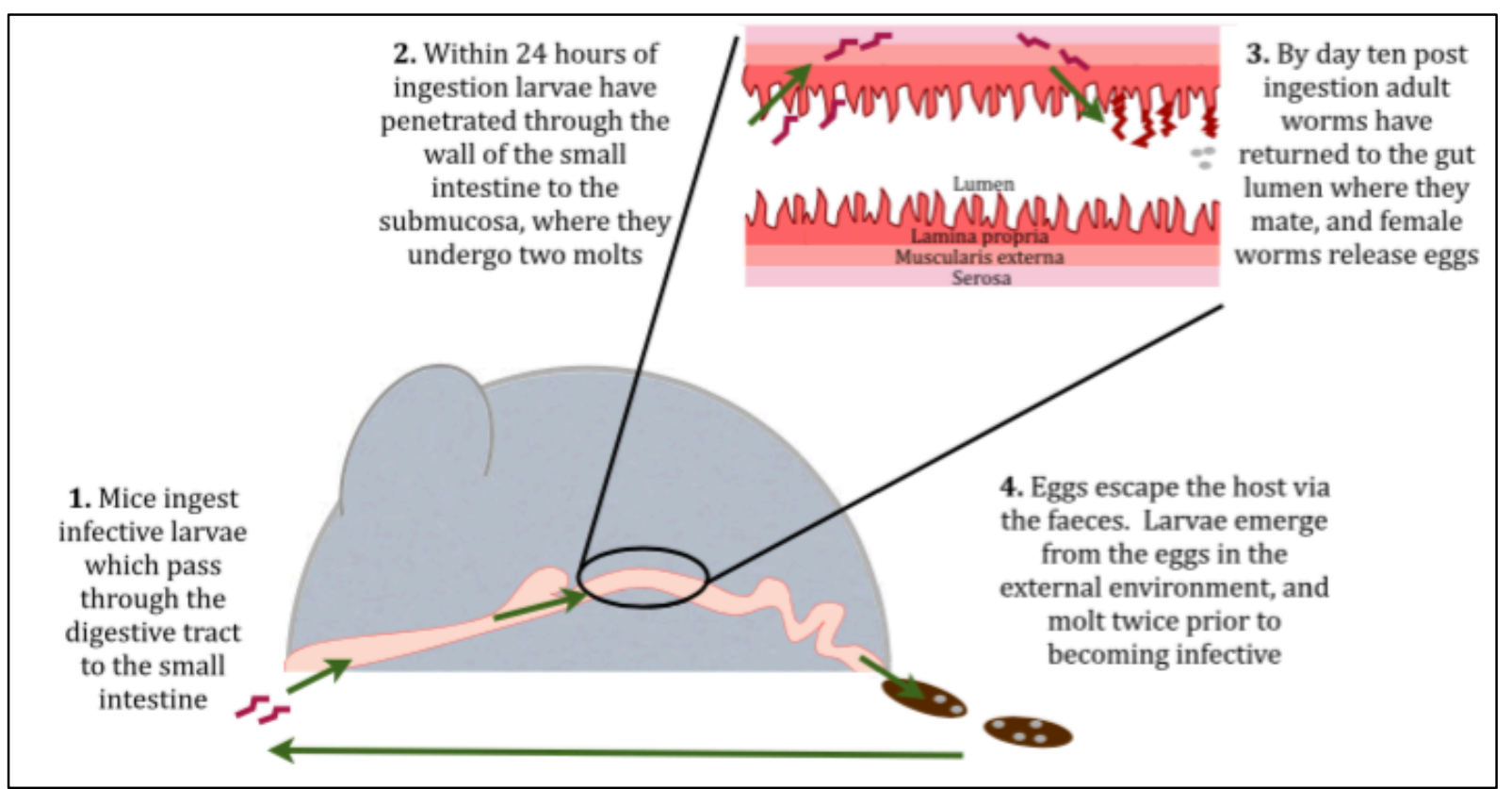

Figure 1.2: The lifecycle of $H$. polygyrus in mice

Infective L3-stage larvae are ingested by the mice (or administered by oral gavage in an experimental setting) and pass to the small intestine where they penetrate the wall and undergo two moults whilst encysted in the submucosa. Adult worms (8-10 days post-infection) return to the gut lumen where they coil around the villi and mate, with the eggs released in the host faeces. Once outside the host, the eggs hatch and the larvae undergo two moults to become infective larvae (Reynolds, L.A., Filbey, K.J. \& Maizels, R.M. Immunity to the model intestinal helminth parasite Heligmosomoides polygyrus. Semin Immunopathol 34, 829-846 (2012)). ${ }^{79}$ 


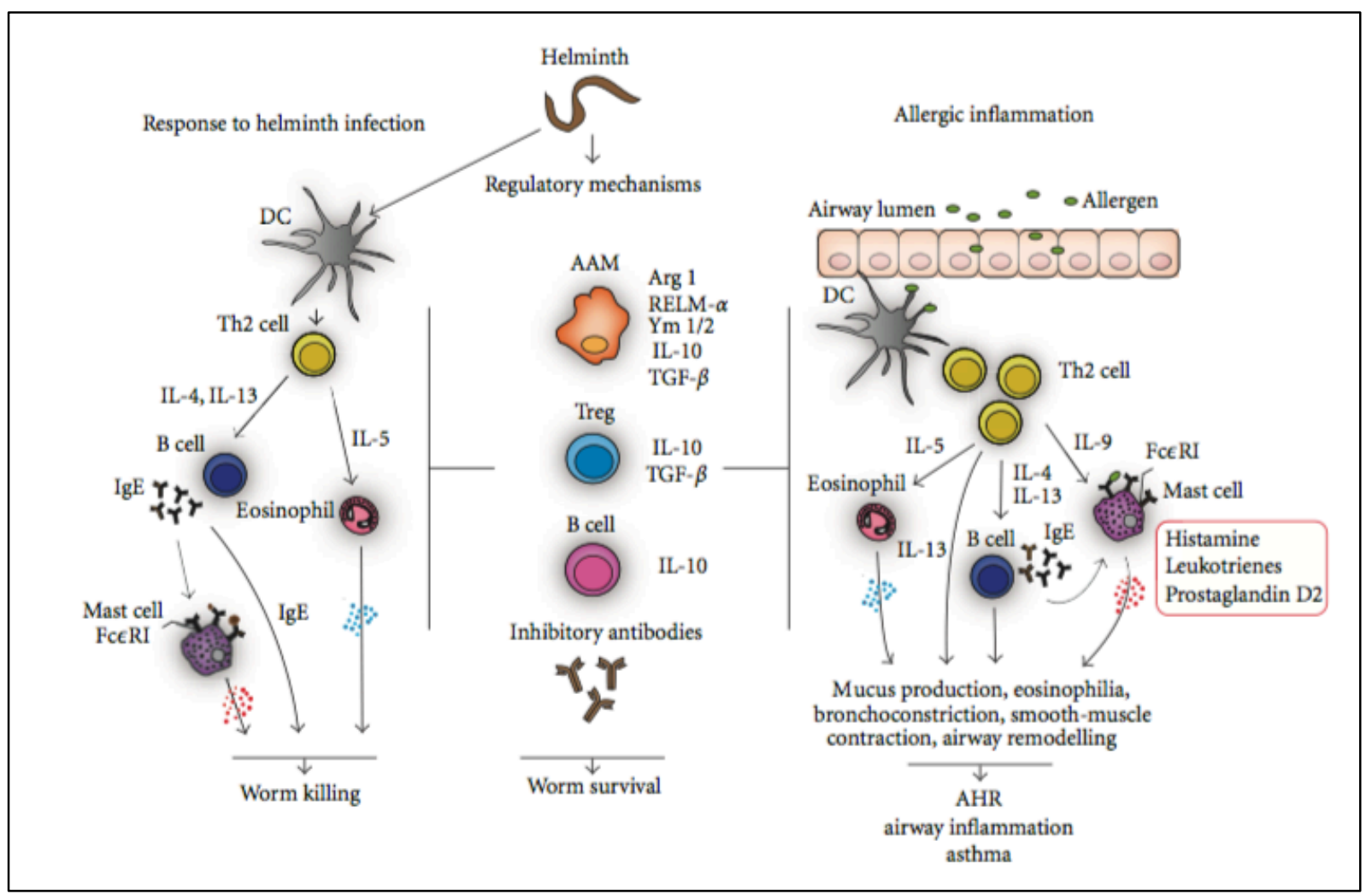

Figure 1.3: Immunomodulation by helminth parasites

Helminths induce a Th2 response consisting of Th 2 cells, B cells, eosinophils, mast cells, neutralizing antibodies and Th2 cytokines IL-4, IL-5, and IL-13. However, they also actively induce the regulatory mechanisms of the Th2 response consisting of AAMs (or M2 macrophages), Tregs and regulatory B cells. M2 macrophages secrete molecules implicated in wound healing. Tregs secrete IL-10 and TGF $\beta$, and regulatory B cells secrete IL-10 and inhibitory antibodies. The induction of these cells and regulatory molecules leads to suppression of inflammatory immune responses and prolonged worm survival and reproduction. Allergic inflammation presents a similar phenotype to the anti-helminth immune response, and in the lungs (as seen in allergic asthma) can lead to mucus production, accumulation of eosinophils, bronchoconstriction, smooth muscle contraction and airway remodelling. This allergic inflammation is likely to be suppressed due to bystander effects from regulatory mechanisms induced by helminth infection (Daniłowicz-Luebert, E., O'Regan, N.L., Steinfelder, S. \& Hartmann, S. Modulation of specific and allergy-related immune responses by helminths. J Biomed Biotechnol 2011 (2011)). ${ }^{17}$ 


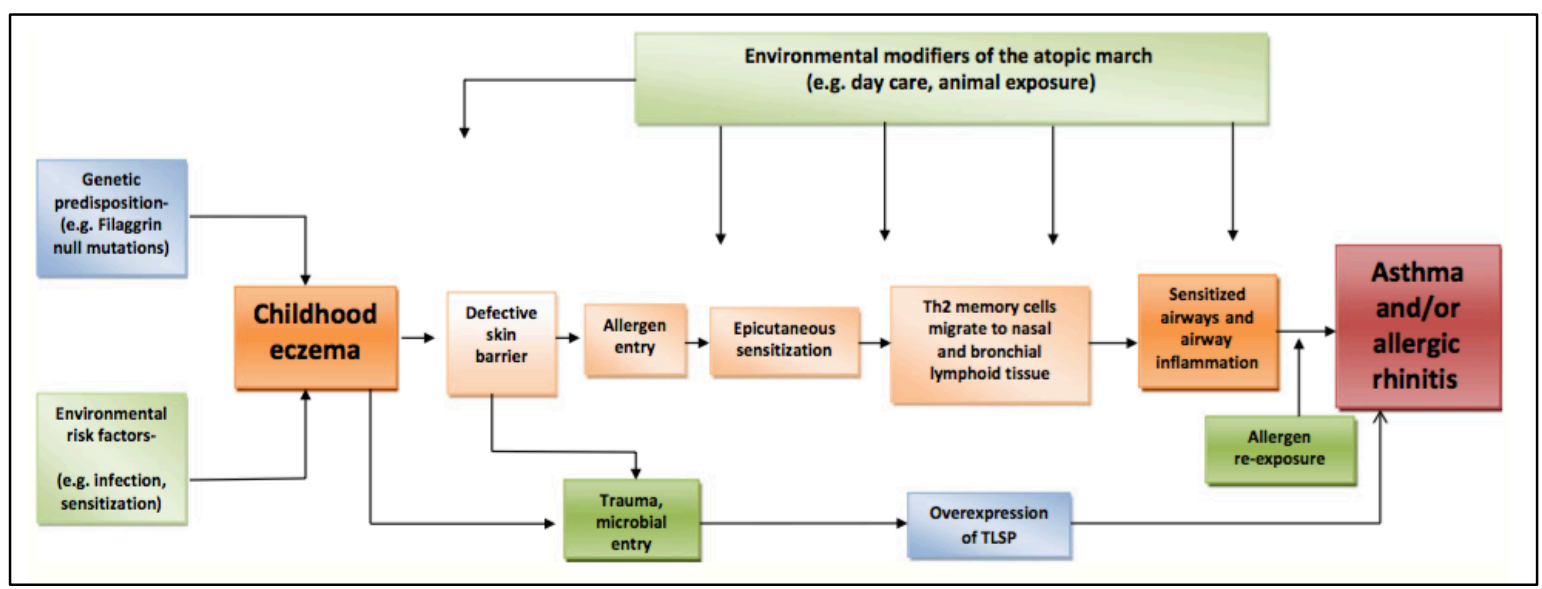

\section{Figure 1.4: The allergic march}

The proposed pathway illustrating the allergic march and the development of asthma and other airway allergies from childhood eczema. A mixture of genetic and environmental factors creates a defective skin barrier allowing entry of, and epicutaneous sensitization by, allergens. The migration of Th2 memory cells from the skin to other lymphoid tissues, such as the nasal and bronchial lymphoid tissue, sensitises the airways, leading to asthma or allergic rhinitis after re-exposure to the same allergen. Furthermore, trauma to the skin barrier triggers the release of TSLP which enhances further allergic responses (Dharmage, S.C. et al. Atopic dermatitis and the atopic march revisited. Allergy 69, 17-27 (2014)). ${ }^{172}$ 
Chapter 2. Material and Methods 


\subsection{Materials}

\subsubsection{Labware}

Product

Beads (stainless steel, $5 \mathrm{~mm}$ ) suitable for tissue lyser

Clear flat-bottom (Nunc) immuno nonsterile 96-well plates

Eppendorf ${ }^{\circledast}$ Safe-Lock microcentrifuge tubes $2 \mathrm{ml}$

Falcon $^{\mathrm{TM}} \mathrm{lml}$ Tuberculin syringes

Falcon $^{\mathrm{TM}} 70 \mu \mathrm{m}$ cell strainer

Falcon $^{\text {TM }}$ conical polystyrene sterile centrifuge tubes, 15 and $50 \mathrm{ml}$

Falcon $^{\mathrm{TM}}$ polystyrene round bottom tubes $5 \mathrm{ml}$

Falcon $^{\mathrm{TM}}$ polystyrene serological pipet 2, 5, 10 and $25 \mathrm{ml}$ In vitro Technologies

Falcon ${ }^{\mathrm{TM}}$ polystyrene sterile clear flat bottom well plates In vitro Technologies $6,12,24$ wells

Falcon $^{\mathrm{TM}}$ polystyrene sterile clear round bottom well plate 96 wells

MicroAmp ${ }^{\circledast}$ optical 96-well reaction plate with barcode

Microtome blades MX35

Plate adhesive seals suitable for qPCR

PrecisionGlide needles 25G

Stainless steel feeding needle 22G

Sterile microtubes 0.2 and $1.7 \mathrm{ml}$ clear
Supplier

Qiagen

Thermofisher Scientific

Sigma

In vitro Technologies

In vitro Technologies

In vitro Technologies

In vitro Technologies

In vitro Technologies

Applied Biosystems

Thermofisher Scientific

Applied Biosystems

BD Biosciences

Total lab systems

Axygen Scientific Inc. 
Sterile pipet tips (certified RNAse free) 10, 20, 200 \& $1000 \mu \mathrm{l}$

Sterile screw top tubes $5 \mathrm{ml}$

Sterile striptubes $0.2 \mathrm{ml}$ clear

Superfrost plus microscope slides

Surgical blade number 10

Tissue-Loc cassettes

Transfer pipette, sterile individually wrapped $\mathrm{lml}$

Ultrafine insulin syringe needle $29 \mathrm{G} 0.3 \mathrm{ml}$
Axygen Scientific Inc.

Thermofisher Scientific

Axygen Scientific Inc.

Thermofisher Scientific

Swann-Morton

Thermofisher Scientific

Interlab

BD Biosciences 


\subsubsection{Reagents}

\begin{tabular}{|c|c|c|}
\hline Reagents & Catalog number & Supplier \\
\hline Accutase & SCR104 & Chemicon \\
\hline Acetone & AC03112500 & Scharlau \\
\hline Bovine serum albumin (BSA) & A7888-100G & Sigma \\
\hline Brefeldin A & B5936-200UL & Sigma \\
\hline Collagenase I & 17018029 & Roche \\
\hline Collagenase IV & C5138-1g & Sigma \\
\hline Compensation beads anti-mouse & 552843 & BD CompBead \\
\hline $\begin{array}{l}\text { Compensation beads anti-rat/anti- } \\
\text { hamster }\end{array}$ & 552845 & BD CompBead \\
\hline $\begin{array}{l}\text { Crushed up whole house dust mite } \\
\text { (Dermatophagoides pteronyssinus) }\end{array}$ & B84 & Greer \\
\hline Cytofix/Cytoperm kit & 554714 & BD Biosciences \\
\hline DEPC-treated $\mathrm{H}_{2} \mathrm{O}$ & AM9906 & Ambion \\
\hline Di-n-butyl phthalate (DBP) & 31244 & Alfa Aesar \\
\hline Dispase II & 04942078001 & Roche \\
\hline DNAse 1 & 1010415900 & Roche \\
\hline $\begin{array}{l}\text { Dulbecco's phosphate-buffered saline } \\
\text { (DPBS) }\end{array}$ & $14190-250$ & Gibco \\
\hline Ethanol (absolute) & N1008622-5A & Pure Science \\
\hline Ethylenediaminetetraacetic acid (EDTA) & $15575-038$ & Invitrogen \\
\hline Fetal Bovine Serum (FBS) & $10091-148$ & Gibco \\
\hline
\end{tabular}




\begin{tabular}{|c|c|c|}
\hline Fluorescein isothiocyanate (FITC) & F7250 & Sigma \\
\hline $\begin{array}{l}\text { FoxP3/ Transcription Factor Staining } \\
\text { Buffer Set }\end{array}$ & $00-552300$ & eBioscience \\
\hline $\begin{array}{l}\text { Hank's Balanced Salt Solution (HBSS) } \\
\text { with Phenol Red and Glucose }\end{array}$ & $14170-161$ & Gibco \\
\hline High Capacity RNA-to-cDNA kit & 4387406 & $\begin{array}{l}\text { Applied } \\
\text { Biosystems }\end{array}$ \\
\hline Ig - HRP secondary antibody & 1010-05 & Southern Biotech \\
\hline Ionomycin & I3909-1ML & Sigma \\
\hline $\begin{array}{l}\text { Iscove's Modified Dulbecco Medium } \\
\text { (IMDM) with Phenol Red, GlutaMAX } \\
\text { mM HEPES buffer and } 3.024 \mathrm{mg} / \mathrm{L} \\
\mathrm{NaHCO}_{3}\end{array}$ & 31980-097 & Gibco \\
\hline Isopropanol & I95-16-500ML & Merck \\
\hline Liberase TM & 05401127001 & Roche \\
\hline Penicillin-streptomycin & 15140122 & Gibco \\
\hline Phorbol 12-myristate 13-acetate (PMA) & 524400 & Calbiochem \\
\hline Phosphate-buffered saline (PBS) powder & $21600-069$ & Gibco \\
\hline Red blood cell (RBC) lysing buffer & R7757-100ml & Sigma \\
\hline RNAlater & AM7021 & Invitrogen \\
\hline $\begin{array}{l}\text { Roswell Park Memorial Institute (RPMI) } \\
1640 \text { Medium with L-glutamine and } \\
\text { Phenol Red }\end{array}$ & $11875-119$ & Gibco \\
\hline Sodium azide $\left(\mathrm{NaN}_{3}\right)$ & S8032-25G & Sigma-Aldrich \\
\hline Sodium chloride $(\mathrm{NaCl})$ & S9888-1KG & Sigma-Aldrich \\
\hline Sphero Accucount beads & ACBP-50-10 & Spherotech \\
\hline
\end{tabular}


Sulfuric acid $\left(\mathrm{H}_{2} \mathrm{SO}_{4}\right)$

Taqman gene expression master mix

TMB substrate

Trizol

Trypan Blue

Tween-20
VWRC102765G

4352042

555214

15596026

15250-061

P1379-500ML
BDH Chemicals

(Analar)

Applied

Biosystems

BD OptEIA

Invitrogen

Gibco

Sigma-Aldrich 


\subsubsection{Buffers and solutions}

Buffers and solutions

Composition

Anaesthetic working solution DPBS

10x stock solution ketamine/xylazine (1:10 dilution in DPBS) provided by the Biomedical Research Unit at the Malaghan Institute

Complete HBSS media (cHBSS) HBSS 500ml

$10 \%$ FCS (50ml)

1\% Pen-Strep (5ml)

Complete IMDM media (cIMDM) IMDM 500ml

$10 \%$ FCS (50ml)

1\% Pen-Strep (5ml)

Complete RPMI media (cRPMI) RPMI 500ml

$10 \%$ FCS (50ml)

1\% Pen-Strep (5ml)

Digestion mix 1: Ear Tissue

Accutase

Dispase II - 3U/ml

Liberase TM - $100 \mu \mathrm{g} / \mathrm{ml}$

Digestion mix 2: Ear Tissue

HBSS

Collagenase IV - $2 \mathrm{mg} / \mathrm{ml}$

DNAse 1 - $100 \mu \mathrm{g} / \mathrm{ml}$

Digestion mix 3: Lymph Node DPBS

Collagenase IV - 1mg/ml

DNAse 1 - 100 $\mu \mathrm{g} / \mathrm{ml}$ 
Digestion mix 4: Lung tissue

DBP-FITC

ELISA blocking buffer

ELISA coating buffer

ELISA wash buffer

FACS buffer

Perm buffer

Perm wash

Stop buffer 1
cIMDM

Collagenase I - $2.5 \mathrm{mg} / \mathrm{ml}$

DNAse 1 - 120 $\mu \mathrm{g} / \mathrm{ml}$

$0.5 \%$ FITC

1:1 DBP/Acetone

lx PBS

$2 \%$ BSA

$500 \mathrm{ml} \mathrm{dH}{ }_{2} \mathrm{O}$

$1.5 \mathrm{~g} \mathrm{Na}_{2} \mathrm{CO}_{3}$

$3 \mathrm{~g} \mathrm{NaHCO}_{3}$

Adjust $\mathrm{pH}$ to 9.6

lx PBS

0.05\% Tween-20

1x PBS 500ml

$0.5 \%$ BSA (2.5g)

$0.05 \% \mathrm{NaN}_{3}(250 \mu \mathrm{l})$

$\mathrm{dH}_{2} \mathrm{O}$

10x Permeabilization buffer from FoxP3/

Transcription Factor Staining Buffer Set (1:10 dilution)

$\mathrm{dH}_{2} \mathrm{O}$

10x Cyto-permeabilization buffer from Cytofix/Cytoperm kit (1:10 dilution)

5mM EDTA 
Stop buffer 2

DPBS

5mM EDTA

Saturated salt solution

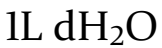

$400 \mathrm{~g} \mathrm{NaCl}$

\subsubsection{Mice}

\subsubsection{Mouse strains}

C57BL/6J mice (Breeding pairs obtained from The Jackson Laboratory): These mice are unable to expel $H$. polygyrus in a primary infection.

$4 C 13 R$ dual reporter mice: these mice express fluorescent reporters for IL-4 (AmCyan) and IL-13 (DS-Red). (Obtained from Dr. William E. Paul, National institute of Allergic Diseases, National Institute of Health)

\subsubsection{Maintenance and ethical approval}

All mice (female and 6-10 weeks of age) were bred in the Biomedical Research Unit at the Malaghan Institute of Medical Research. All procedures were carried out as approved by the Victoria University of Wellington (protocol: 2014R15M). 


\subsubsection{Flow cytometry:}

Antibodies were titrated and the optimal antibody dilution was determined after purchase. Antibody mixes were made up in FACS buffer or perm buffer and vortexed just before use.

\subsubsection{List of used antibodies}

Antibody
Conjugate

\section{Bc16}

CD3

\begin{abstract}
CDs
\end{abstract}
CD4

B

BV786

PerCP
Company

BD Pharmingen

BD Pharmingen

BD Pharmingen

APC-Cy7

FITC

BV605

BUV737

FITC

BV786

$\mathrm{CD} 8 \alpha$

CDIlb

BV786

BD Pharmingen

BD Pharmingen

BD Pharmingen

BD Pharmingen

Made in house

BD Pharmingen

BV650

BD Pharmingen

RM4-5

GK1.5

RM4-5

RM4-5

GK1.5

2.43

53-6.7

$\mathrm{Ml} / 70$

FITC

BD Pharmingen

$\mathrm{Ml} / 70$

APC-Cy7

BioLegend

N418

BUV395

BD Pharmingen

M1/70

CD1lc

FITC

BioLegend

N418

PE-Cy7

BD Pharmingen

HL3

BUV395

BD Pharmingen

HL3

\section{CD19}

APC-H7

BD Pharmingen

ID3

Clone 


\begin{tabular}{|c|c|c|c|}
\hline \multirow[t]{2}{*}{ CD25 } & FITC & BD Pharmingen & 7D4 \\
\hline & BV421 & BD Pharmingen & 7D4 \\
\hline CD44 & APC & BD Pharmingen & IM7 \\
\hline \multirow[t]{4}{*}{ CD45 } & BV510 & BD Pharmingen & 30.F11 \\
\hline & BUV395 & BD Pharmingen & 30.F11 \\
\hline & APC & BD Pharmingen & 30.F11 \\
\hline & APC-Cy7 & BD Pharmingen & 30.F11 \\
\hline \multirow[t]{4}{*}{ CD45R (B220) } & BV786 & BD Pharmingen & RA3-6B2 \\
\hline & FITC & BD Pharmingen & RA3-6B2 \\
\hline & BV650 & BD Pharmingen & RA3-6B2 \\
\hline & PE-CF594 & BD Horizon & RA3-6B2 \\
\hline \multirow[t]{2}{*}{ CD49b (DX5) } & PE-Cy7 & eBioscience & DX5 \\
\hline & FITC & BioLegend & DX5 \\
\hline CD62L & FITC & BD Pharmingen & MEL-14 \\
\hline CD64 & APC & BioLegend & $\mathrm{X} 54-5 / 7.1$ \\
\hline CD69 & PE-Cy7 & BD Pharmingen & $\mathrm{H1.2F3}$ \\
\hline CD86 & $\mathrm{PE}$ & BD Pharmingen & GL1 \\
\hline CD90.2 & BV605 & BD Pharmingen & $53-2.1$ \\
\hline CD103 & APC & eBioscience & 2E7 \\
\hline CD117 (Ckit) & BV421 & BD Pharmingen & 2B8 \\
\hline \multirow[t]{2}{*}{ CD278 (ICOS) } & BV421 & BioLegend & $\mathrm{C} 398.4 \mathrm{~A}$ \\
\hline & AF647 & BioLegend & C398.4A \\
\hline
\end{tabular}




\begin{tabular}{|c|c|c|c|}
\hline CD326 & BV711 & BD Pharmingen & G8.8 \\
\hline F4/80 & FITC & eBioscience & BM8 \\
\hline FcgIII/IR (block) & & Made in house & $2.4 \mathrm{G} 2$ \\
\hline \multirow[t]{3}{*}{ FceRI $\alpha$} & Biotin & eBioscience & MAR-1 \\
\hline & FITC & eBioscience & MAR-1 \\
\hline & AF647 & eBioscience & MAR-1 \\
\hline FoxP3 & $\mathrm{PE}$ & eBioscience & NRRF-30 \\
\hline \multirow[t]{2}{*}{ GATA3 } & AF647 & BD Pharmingen & L50-823 \\
\hline & BV421 & BD Pharmingen & L50-823 \\
\hline I-A/I-E (MHCII) & Pacific Blue & BioLegend & M5/114.15.2 \\
\hline IL-4 & BV421 & BD Pharmingen & $11 B 11$ \\
\hline IL-10 & $\mathrm{PE}$ & BD Pharmingen & JES5-16E3 \\
\hline IL-13 & eF660 & eBioscience & eBiol3A \\
\hline KLRG1 & APC & BD Pharmingen & $2 \mathrm{Fl}$ \\
\hline Ly6G & BV711 & BD Pharmingen & $1 \mathrm{~A} 8$ \\
\hline NK1.1 & FITC & BD Pharmingen & PK136 \\
\hline Relma & Biotin & AbCam & rabbit pAb \\
\hline Siglec F & PE-CF594 & BD Horizon & E50-2440 \\
\hline ST2 & PerCP-eF710 & eBioscience & RMST2-2 \\
\hline \multirow[t]{2}{*}{ Streptavidin } & BV605 & BD Pharmingen & \\
\hline & PE-Cy7 & BD Pharmingen & \\
\hline TCR $\beta$ & BV605 & BD Pharmingen & H57-597 \\
\hline
\end{tabular}




\subsubsection{Viability dyes}

4,6-diamidino-2-phenylindole, dihydrochloride (DAPI), purchased from Invitrogen, was prepared in $\mathrm{H}_{2} \mathrm{O}$ at a concentration of $200 \mu \mathrm{g} / \mathrm{ml}$. DAPI was added to the samples just before analysis at a dilution of 1:5,000 in PBS.

Live/dead fixable blue, purchased from Invitrogen, was reconstituted in 50 $\mu$ l DMSO from the same kit and diluted 1:1000 in PBS. 200 $\mu$ l was added per well and cells were incubated for 20 minutes in the dark at $4^{\circ} \mathrm{C}$.

\subsection{6 qPCR primers}

\begin{tabular}{llll}
\multicolumn{1}{c}{ Gene } & Dye & \multicolumn{1}{c}{ Assay ID } & Company \\
\hline Foxp3 & FAM-MGB & Mm00475162_ml & Thermofisher Scientific \\
Gapdh & VIC-MGB & NM_008084.2 & Thermofisher Scientific \\
Il4 & FAM-MGB & Mm00445259_ml & Thermofisher Scientific \\
Illo & FAM-MGB & Mm01288386_ml & Thermofisher Scientific \\
Ill3 & FAM-MGB & Mm00434204_ml & Thermofisher Scientific \\
Il33 & FAM-MGB & Mm00505403_ml & Thermofisher Scientific \\
Tgf $\boldsymbol{T}$ & FAM-MGB & Mm01178820_ml & Thermofisher Scientific \\
Tslp & FAM-MGB & Mm01157588_ml & Thermofisher Scientific
\end{tabular}




\subsection{Methods}

\subsubsection{Mouse treatments}

\subsubsection{Intragastric (IG) administration of infective $L 3 H$. polygyrus larvae}

Viability and concentration of L3 $H$. polygyrus larvae were assessed by light microscopy. Mice were given $200 \mathrm{~L} 3$ larvae in $200 \mu \mathrm{H}_{2} \mathrm{O}$ via oral gavage using a stainless steel $22 \mathrm{G}$ feeding needle.

\subsubsection{Intradermal injection of mashed-up whole HDM}

Mice were anaesthetized with $200 \mu$ l of anaesthetics working solution by intraperitoneal (IP) injection with a 25G needle. Mice were given an ID injection in both ears with a 29G insulin needle containing $200 \mu \mathrm{g}$ of mashed up HDM in $30 \mu \mathrm{l}$ sterile PBS.

\subsubsection{Contact hypersensitivity model of DBP-FITC}

Mice were anaesthetized with $200 \mu$ l anaesthetic working solution by IP injection with a $25 \mathrm{G}$ needle. The hair on the lower part of the abdominal skin was removed by shaving with a number 10 surgical blade, one week before sensitization. Mice were sensitized on the abdominal skin on days 0 and 2 by epicutaneous application of $40 \mu \mathrm{l}$ of DBP-FITC. On day 6, baseline ear thickness was measured with a micrometer (Micromaster, Capa System) and baseline trans-epidermal water loss (TEWL) was measured with TEWL equipment from Dermalab (Dermalab TEWL open chamber units), followed by challenging the mice with $20 \mu \mathrm{l}$ of DBP-FITC on one ear and $20 \mu \mathrm{l}$ of vehicle (DBP) on the other ear. From day 7-13, ear thickness and TEWL was measured and the change from baseline was calculated. On day 8, 2 mice from each group were sacrificed and ear tissue was harvested for histology. On day 14 , remaining mice were sacrificed. 


\subsubsection{Topical application of DBP-FITC for DC migration}

Mice were anaesthetized with $200 \mu$ l anaesthetic working solution by IP injection with a 25G needle. Mice were treated with $20 \mu$ l of DBP-FITC on both ears on day 0 . On day 2 , mice were sacrificed and auricular lymph nodes from all mice, and guts from infected mice were harvested.

\subsubsection{Topical application of DBP-FITC for T cell responses}

Mice were anaesthetized with $200 \mu$ l anaesthetic working solution by IP injection with a $25 \mathrm{G}$ needle. Mice were treated with $20 \mu \mathrm{l}$ of DBP-FITC on both ears on day 0 and day 2. Mice were sacrificed and auricular lymph nodes from all mice, and guts from infected mice were harvested on day 7 and 9.

\subsubsection{Euthanasia}

Mice were sacrificed by lethal injection of anaesthetic (200 $\mu$ l 10x stock solution) and cervical dislocation, or $\mathrm{CO}_{2}$ asphyxiation and/or cervical dislocation.

\subsubsection{Tissue harvest and processing}

\subsubsection{Lymph nodes}

Auricular LNs were carefully removed from euthanized mice and placed in DPBS until processed.

Generating single cell suspensions from lymph nodes for analysis of immune cells Isolated LNs were mashed through a $70 \mu \mathrm{m}$ cell strainer using the rubber end of a $\mathrm{lml}$ syringe. The strainer was washed with $5 \mathrm{ml}$ cRPMI. Samples were centrifuged at 1300 rounds per minute (RPM) for 5 minutes; the supernatant was discarded and the pellet was resuspended with $1 \mathrm{ml}$ cRPMI for counting. 
Generating single cell suspensions from lymph nodes for analysis of dendritic cells

Isolated LNs were cut in half and digested in $\mathrm{lml}$ of digestion buffer (digestion mix 7

for LNs) for $30 \mathrm{~min}$ at room temperature (RT) on a rotator. After digestion $2 \mathrm{ml}$ of DPBS with 5mM EDTA was added to stop any further digestion. LNs were mashed through a $70 \mu \mathrm{m}$ cell strainer using the rubber end of a $1 \mathrm{ml}$ syringe, centrifuged at 1300 RPM for 5 minutes and resuspended in 1ml of DPBS with 5mM EDTA for counting.

\subsubsection{Ear tissue}

Ears that were harvested from mice were kept either in DPBS for use in flow cytometry, or in RNAlater for use in quantitative PCR (qPCR).

\section{Digestion mix 1}

Ears used for flow cytometry were split into dorsal and ventral halves and placed dermis side down in a 12 -well plate in $1 \mathrm{ml}$ of digestion enzyme mix per ear. Ears were incubated for $20 \mathrm{~min}$ in a shaking incubator (100 RPM) at $37^{\circ} \mathrm{C}$. After incubation ears were chopped up into small pieces and incubated for another 20 minutes in a shaking incubator (100 RPM) at $37^{\circ} \mathrm{C}$. After incubation, ear tissue was filtered through a $70 \mu \mathrm{m}$ cell strainer using the rubber end of a $1 \mathrm{ml}$ syringe and washed with $10 \mathrm{ml}$ of cRPMI.

\section{Digestion mix 2}

Ears used for flow cytometry were split into dorsal and ventral halves and immediately chopped up into small pieces in $5 \mathrm{ml}$ tubes and incubated for 30 minutes in a shaking incubator (150 RPM) at $37^{\circ} \mathrm{C}$ with $1 \mathrm{ml}$ digestion buffer per ear. After incubation, lml HBSS stop buffer per ear was added to stop digestion. Ear tissue was filtered through a $70 \mu \mathrm{m}$ cell strainer using the rubber end of a $\mathrm{lml}$ syringe and washed with $10 \mathrm{ml}$ of cRPMI.

After digestion, cells were centrifuged for 10 minutes at 1300 RPM, supernatant was removed and cells were resuspended at $1 \mathrm{ml}$ for counting. After counting cells, $100 \mu \mathrm{l}$ 
of the cell samples was kept in a $1.7 \mathrm{ml}$ microtube for counting by flow cytometry, the rest of the samples was used for analysis by flow cytometry

\subsubsection{Parasitology}

The small intestine and cecum were harvested from euthanized mice and placed on ice until processed.

\section{Life cycle and maintenance}

Mice are orally infected with infective L3 larvae. After infection, the larvae first move into the gastric mucosa of the stomach. The larvae emerge after 36 hours and proceed to the duodenum and burrow close to the longitudinal muscle layer of the gut. Adult parasites emerge after 8 days into the lumen after which they mate and produce eggs 9 days after infection, which can last for several months. ${ }^{178}$

H. polygyrus larvae lifecycles were maintained by the Malaghan Institute according to protocol. ${ }^{178}$ Infective $\mathrm{L} 3$ larvae were kept in $\mathrm{H}_{2} \mathrm{O}$ at $4^{\circ} \mathrm{C}$ at a concentration of 200 L3 larvae per $200 \mu \mathrm{l}$.

\section{Adult worm count}

The small intestine was sliced open longitudinally and hung over a parafilm strip in a $50 \mathrm{ml}$ falcon tube filled with PBS and placed overnight in a half-filled $37^{\circ} \mathrm{C}$ water bath creating a thermal gradient. Viable adult worms actively migrate from the gut to the bottom of the $50 \mathrm{ml}$ tube. The next day adult worms were collected and counted under a dissecting microscope.

\section{Egg count}

The contents of the cecum were transferred to a $5 \mathrm{ml}$ tube and dissolved in $2 \mathrm{ml}$ $\mathrm{dH}_{2} \mathrm{O}$. After a few hours, $2 \mathrm{ml}$ of saturated salt solution was added and eggs were counted under a light microscope using a McMaster egg counting chamber. 


\subsubsection{Serum}

Mice were euthanized through a lethal dose of anaesthetics. Whole blood was obtained through brachial bleed and the blood was collected in a $1.7 \mathrm{ml}$ microtube. Blood was left to clot overnight at $4^{\circ} \mathrm{C}$ and then centrifuged for 10 minutes at 2000 RPM. Serum was collected in a new tube and centrifuged again for 10 minutes at 2000 RPM to remove all RBCs. Serum was collected in a new tube and frozen at $70^{\circ} \mathrm{C}$ until used for analysis by enzyme-linked immunosorbent assay (ELISA).

\subsubsection{Whole blood}

Mice were euthanized through a lethal dose of anaesthetics. Whole blood was obtained through brachial bleed and the blood was collected in a sterile FACS tube with lml DPBS with 10mM EDTA and mixed thoroughly. Blood was lysed twice for 4 minutes with $5 \mathrm{ml}$ RBC lysing buffer and resuspended in media for analysis by flow cytometry.

\subsubsection{Lung}

Mice were euthanized by cervical dislocation. Lungs were harvested and digested using the digestion mix 8 for lung tissue. Lungs were chopped up into small pieces in a 6-well plate and $5 \mathrm{ml}$ of digestion mix was added. Plates were incubated in a $37^{\circ} \mathrm{C}$ shaking incubator for 1 hour at 100 RPM. The cells were filtered through a $70 \mu \mathrm{m}$ cell strainer using the rubber end of a $1 \mathrm{ml}$ syringe. The strainer was washed with 10ml cIMDM. Samples were centrifuged at 1400 RPM for 5 minutes; supernatant was discarded and the cells were lysed in $4 \mathrm{ml} \mathrm{RBC} \mathrm{lysing} \mathrm{buffer} \mathrm{for} 4$ minutes. Cells were washed with $10 \mathrm{ml}$ cIMDM and centrifuged at 1300 RPM for 5 minutes and resuspended in 5ml cIMDM for cell count. 


\subsubsection{Cell count}

Cells were counted under a light microscope using trypan blue for exclusion of dead cells. Samples were diluted 1:1 in Trypan blue dye and pipetted onto a haemocytometer for counting. RBCs and cells that had taken up the dye were excluded from the cell count. Samples are centrifuged and resuspended to a concentration between $10 \times 10^{6-} 25 \times 10^{6}$ cells $/ \mathrm{ml}$.

\subsubsection{Cell staining}

\subsubsection{Cell surface staining}

$1 \times 10^{6}-5 \times 10^{6}$ cells/well were added to a round bottom 96-well plate, centrifuged for 2 minutes at 1300 RPM and washed twice in PBS. Cells were incubated in 50 $\mu$ l of 1:400 dilution of Fc block (2.4G2) in FACS buffer for 10 minutes at $4^{\circ} \mathrm{C}$. After incubation, the cells were washed once in $150 \mu$ l of FACS buffer. $30 \mu$ l of cell surface antibody mix made up in FACS buffer was added per well, and the cells were incubated in the dark at $4^{\circ} \mathrm{C}$ for 20 minutes. Cells were washed twice with FACS buffer, and incubated for another 20 minutes at $4{ }^{\circ} \mathrm{C}$ with the secondary antibody and/or resuspended in $200 \mu$ l FACS buffer and transferred to microtubes for analysis by flow cytometry.

\subsubsection{Intracellular staining of transcription factors}

After staining with cell surface antibodies, cells were permeabilized with transcription factor fix/perm kit for $30 \mathrm{~min}$ at RT or ON at $4^{\circ} \mathrm{C}$. Cells were washed twice in perm buffer and $30 \mu \mathrm{l}$ of intracellular antibody mix made up in perm buffer was added per well. Cells were incubated ON (if permeabilized for 30 minutes at RT) or for 20 minutes at $4^{\circ} \mathrm{C}$ (if permeabilized $\mathrm{ON}$ at $4^{\circ} \mathrm{C}$ ). Cells were washed twice in perm buffer, resuspended in $200 \mu$ l of FACS buffer, and transferred to microtubes for analysis by flow cytometry. 


\subsubsection{Intracellular cytokine staining}

Isolated lymphocytes were stimulated for 4 hours at $37^{\circ} \mathrm{C}$ in cRPMI with PMA (50ng/ml), Ionomycin $(1 \mu \mathrm{g} / \mathrm{ml})$, and Brefeldin A $(10 \mu \mathrm{g} / \mathrm{ml})$. After stimulation, cells were stained with cell surface staining mix. After washing, cells were permeabilized for 30 minutes at $4^{\circ} \mathrm{C}$ in Cytofix/Cytoperm solution (BD Biosciences). Cells were left overnight in perm wash at $4^{\circ} \mathrm{C}$ and stained the next day with intracellular cytokine staining mix in perm wash for 20 minutes at $4^{\circ} \mathrm{C}$. Cells were resuspended in $200 \mu \mathrm{l}$ FACS buffer for analysis by flow cytometry.

\subsubsection{Viability dye staining}

DAPI was added either just before analysis in a 1:5000 dilution, or live/dead fixable blue staining was added in a 1:1000 dilution for 20 minutes before staining with cell surface antibodies.

\subsubsection{Flow cytometry}

\subsubsection{Controls}

Controls used for experiments included: unstained cells, live/dead single stained cells, fluorescence minus one (FMO) stained cells, and single stained compensation beads.

\subsubsection{Acquisition and analysis}

Samples were acquired on either the LSRII or the Fortessa using FACSdiva software. At least 100,000 events were recorded for lymph node and skin samples. At least $1 \times 10^{6}$ events were recorded for DC experiments. Data was analysed using FlowJo software. 


\subsubsection{Counting cells by flow cytometry}

After cell count, $100 \mu \mathrm{l}$ of cell samples was placed in a $1.7 \mathrm{ml}$ microtube. Cells were centrifuged at 1300 RPM for 3 minutes and resuspended in 100 $\mu$ l FACS buffer for flow cytometry. Just before analysis, $50 \mu \mathrm{l}$ of Sphero Accucount beads (containing about 50,000 beads) were added to the mix and $5 \mu$ l of 1:1000 dilution of DAPI. One sample containing only beads was added as a control. At least 100,000 events were collected on the LSRII.

\subsubsection{Quantitative PCR}

\subsubsection{RNA extraction}

Ear tissue was removed from RNAlater and placed in a cliplock tube in $500 \mu$ Trizol. Ears were roughly chopped and either frozen in $-70^{\circ} \mathrm{C}$ freezer or used immediately for RNA extraction. One stainless steel bead $(5 \mathrm{~mm})$ was added to the tubes and samples were homogenized 2x 2 minutes in a tissue lyser (Tissue Lyser II, Qiagen). Another 500 $\mu \mathrm{l}$ Trizol was added after homogenization. The samples were centrifuged at $12,000 \mathrm{~g}$ for 10 minutes at $4^{\circ} \mathrm{C}$ and the supernatant was transferred to a sterile $1.7 \mathrm{ml}$ microtube. The samples were left to rest for 5 minutes at RT, after which $200 \mu$ l of chloroform was added per sample. The samples were shaken and incubated for 2-3 minutes at RT. Samples were centrifuged at 12,000g for 15 minutes at $4^{\circ} \mathrm{C}$ and the aqueous phase was transferred to a new sterile $1.7 \mathrm{ml}$ microtube. $500 \mu \mathrm{l}$ of isopropanol was added to the aqueous phase and the samples were incubated for 10 minutes at RT. Samples were centrifuged at $12,000 \mathrm{~g}$ for 10 minutes at $4^{\circ} \mathrm{C}$ and the supernatant was removed leaving a RNA pellet at the bottom of the tube. The samples were washed with $1 \mathrm{ml} 70 \%$ ethanol, briefly vortexed and centrifuged at $7500 \mathrm{~g}$ for 5 minutes at $4^{\circ} \mathrm{C}$. The supernatant was removed and the pellet was left to air dry for 5-10 minutes. The RNA pellet was resuspended in $20 \mu \mathrm{l}$ DEPC-treated $\mathrm{H}_{2} \mathrm{O}$ and incubated in a heat block set at $60^{\circ} \mathrm{C}$. RNA concentrations were measured using a spectrophotometer (ND-1000, Nanodrop). Samples were stored at $-70^{\circ} \mathrm{C}$. 


\subsubsection{RNA to cDNA conversion}

The high capacity RNA-to-cDNA kit (Applied Biosystems) was used for RNA to cDNA conversion, following the manufacturer's protocol. Per sample: $10 \mu \mathrm{l}$ of $2 \mathrm{x}$ RT buffer, $1 \mu$ l of 20x enzyme mix, and up to $9 \mu$ l of RNA sample (up to $2 \mu \mathrm{g}$ of RNA) was added to $0.2 \mathrm{ml}$ striptubes, DEPC-treated $\mathrm{H}_{2} \mathrm{O}$ was added when necessary to increase the volume to $20 \mu \mathrm{l}$. The tubes were spun down briefly to remove air bubbles and the tubes were placed in a thermo cycler (Veriti 96-well Thermo Cycler, Applied Biosystems) and incubated for 60 minutes on $37^{\circ} \mathrm{C}, 5$ minutes on $90^{\circ} \mathrm{C}$ to stop the reaction and held at $4^{\circ} \mathrm{C}$. cDNA was diluted to a concentration of $25 \mathrm{ng} / \mathrm{ml}$ in DEPC-treated $\mathrm{H}_{2} \mathrm{O}$ and stored in the freezer at $-20^{\circ} \mathrm{C}$.

\subsubsection{Quantitative PCR}

$4 \mu \mathrm{l}$ of cDNA sample (100ng) was added to Optical 96 plates with barcode. Samples were run in duplicate including an $\mathrm{H}_{2} \mathrm{O}$ sample in duplicate as a control. PCR reaction mixes were prepared: per reaction $1 \mu l$ of $20 x$ Taqman gene expression primers were added with $1 \mu \mathrm{l}$ of Gapdh as housekeeping gene, $10 \mu \mathrm{l}$ of $2 \mathrm{x}$ Taqman Gene Expression Master Mix and $4 \mu \mathrm{l}$ of DEPC-treated $\mathrm{H}_{2} \mathrm{O}$. $16 \mu \mathrm{l}$ of this reaction mix was added per well. The plate was covered with a plate adhesive seal and spun down briefly to remove air bubbles. PCR was run and analysed on Applied Biosystems' Quantstudio 7 (Applied Biosystems).

\subsubsection{Serum ELISA}

\subsubsection{House dust mite specific total $\mathrm{lg}$}

Clear 96-well ELISA plates (NUNC) were coated with $1 \mu \mathrm{g} / \mathrm{ml}$ mashed up HDM in pH 9.6 carbonate buffer $(50 \mu l$ per well) and incubated overnight in the fridge. The next day, plates were washed $5 \mathrm{x}$ in wash buffer and moisture flicked off. Plates were blocked with $200 \mu \mathrm{l}$ per well of $2 \%$ BSA in PBS for 2 hours in a $37^{\circ}$ incubator. Serum was defrosted slowly on ice and 1:50 dilutions were made up in block. Samples were serially diluted in 3-fold dilution steps across 7 wells and plates were incubated 
overnight in the fridge. The next day, plates were washed again and $50 \mu$ l block with $\alpha$ Ig detection antibody in a 1:6000 dilution. Plates were incubated for 60 minutes in a $37^{\circ} \mathrm{C}$ incubator. Plates were washed $5 \mathrm{x}$ in wash buffer, and another $5 \mathrm{x}$ in PBS without Tween-20. 100 $\mu$ TMB substrate was added per well, and the reaction was stopped by adding $50 \mu \mathrm{l}$ of $1 \mathrm{M} \mathrm{H}_{2} \mathrm{SO}_{4}$ per well. ELISA plates were read at $450 \mathrm{~nm}$ using an ELISA plate reader (Infinite M1000PRO, Tecan). Titre was calculated as the reciprocal serum dilution at which the dilution curve passed $50 \%$ of the maximum optical density (OD) of the whole plate.

\subsubsection{Statistical analysis}

All statistical analyses were performed using Prism 7 (Graphpad Software Inc.). First, a Shapiro-Wilk test for normality was used to determine normal distribution of samples within a group. If samples passed the Shapiro-Wilk test for normality, for the comparison of 2 groups, an unpaired T-test was used. If samples did not pass the Shapiro-Wilk test for normality, for the comparison of 2 groups, a Mann-Whitney test was used. When more than two groups were compared, a one-way ANOVA was used with a Bonferonni's multiple comparison test. $\mathrm{P}<0.05$ was considered as significant.

Symbols represent individual samples and error bars represent mean \pm the standard error of the mean (SEM). 


\section{Chapter 3. The effect of $\boldsymbol{H}$.}

\section{polygyrus infection on systemic Th2 responses in mice}




\subsection{Introduction}

$H$. polygyrus induces a local Th2 response in the gut resulting in expansion of immune cells in the draining LN throughout infection. While $H$. polygyrus has an entirely enteric lifecycle within the host, previous studies have shown an expansion of IL- $4^{+}$Th2 cells in other tissues such as blood, spleen and lung. ${ }^{179}$ Furthermore, there was also an increased $\mathrm{IL}-4^{+}$population of innate cells such as eosinophils and mast cells. $^{179}$

In the skin, overexpression of IL-4 cannot induce an AD phenotype by itself, but is seen to alter skin homeostatic conditions and induce some processes also seen in AD. ${ }^{166,} 180$ These include increased collagen deposition, keratinocyte hyperproliferation and mast cell accumulation leading to loss of dermal fat tissue, and impaired migration of Langerhans cells. ${ }^{180}$ However, under these conditions no influx of pro-inflammatory cells such as Th2 cells, eosinophils or neutrophils was noted. ${ }^{180}$ Furthermore, overexpression of IL- 4 had a negative influence on wound healing, and wounds in IL-4 transgenic mice showed increased expression of inflammatory cytokines/chemokines and infiltration of inflammatory cells. ${ }^{166}$ However, IL-4 also induces differentiation of AAMs through STAT6, which suppresses Thl/Thl7 responses as well as Th2 cell function through expression of PD-L1, secretion of anti-inflammatory cytokines IL-10 and TGF $\beta$, and enhancing differentiation of Tregs through a retinoic acid dependent mechanism. ${ }^{44,110-112}$ These studies indicate that IL-4 can affect skin in the steady-state and induce AAMs with anti-inflammatory properties, but by itself cannot induce AD.

IL-13 is a pro-inflammatory Th2 cytokine and is constitutively expressed in the skin by intraepithelial cells such as ILC2s. While IL-13 is implicated in the pathogenesis of $\mathrm{AD}$, it is also important for maintaining skin homeostasis and lack of IL-13 leads to decreased skin barrier integrity and impaired wound healing. ${ }^{159,} 181$ This demonstrates IL-13 also has a protective function in the skin under homeostatic conditions.

For this section, 4Cl3R dual-reporter mice were used (originally provided by Dr. W.E. Paul, NIH) to investigate the effect of $H$. polygyrus infection on the activation 
of IL-4 and IL-13 producing immune cells in the skin. These mice were genetically constructed via insertion of a bacterial artificial chromosome and express the fluorescent protein AmCyan under the control of IL-4 and DS-Red under the control of IL-13. ${ }^{149,182}$ The use of these mice allows for the detection of canonical Th2 cytokines IL-4 and IL-13 without the need for in vitro stimulation and intracellular cytokine staining. 


\subsection{Aims}

Previous studies have shown that $H$. polygyrus infection leads to the expression of IL-4 by $\mathrm{T}$ cells and innate cells in the spleen and lung. However, there is no data demonstrating this in skin tissue. Furthermore, as IL-13 is an important cytokine in the Th2 response and also has a protective role in the skin, it could be increased after helminth infection. Therefore, this chapter summarises experiments investigating the effects of $H$. polygyrus in mice on immune cell and cytokine profiles in organs distal to the gut.

Specific aims:

1) To assess if there is an induction of IL-4 in various tissues (MLN, whole blood, lung tissue and ear skin tissue) during infection with $H$. polygyrus, and to assess what immune cells (macrophages, eosinophils, neutrophils, B cells, T cells or ILC2s) are producing IL-4.

2) To assess if there is an induction of IL-13 in these tissues (MLN, whole blood, lung tissue and ear skin tissue) during infection with $H$. polygyrus, and to assess what immune cells (macrophages, eosinophils, neutrophils, B cells, T cells or ILC2s) are producing IL-13. 


\subsection{Results}

For this section, 4C13R mice were used to look at IL-4 and IL-13 expression. Mice were infected with 200 L3 infective larvae 14 days before harvesting tissues. To determine the effects of $H$. polygyrus in mice in various organs, four different tissues were studied: MLN, whole blood, lung tissue and ear skin tissue.

H. polygyrus infection caused expansion of immune cells in the MLN but not in the other tissues assayed (data not shown). Different innate and adaptive immune cells were identified within $\mathrm{CD}^{+} 5^{+}$cells (supplementary figure 1) by flow cytometry:

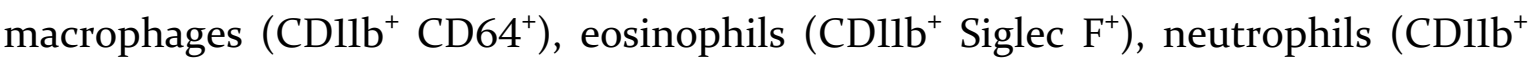
Ly6G $\left.{ }^{+}\right)$, ILC2s ( $\left.\mathrm{Lin}^{-} \mathrm{B} 220^{-} \mathrm{CD}^{-} \mathrm{ICOS}^{+}\right), \mathrm{B}$ cells $\left(\mathrm{B} 22 \mathrm{O}^{+}\right)$and T cells $\left(\mathrm{TCR} \beta^{+} \mathrm{CD} 4^{+}\right)$. Lineage markers used to negatively gate for ILC2s were: CD8, CD1lb, CD1lc, F4/80, DX5 and FceRI. For these immune subsets, the frequency of cells producing IL-4 and/or IL-13 was determined by flow cytometric analysis of the specific reporter (supplemental figure 2, figure 3.1).

In the MLN, IL-4 production was significantly increased in infected mice of which the main producers are T cells (figure 3.1A). Although macrophages and eosinophils also produced IL-4 in this site, the difference between naive and $H$. polygyrusinfected groups was not significant. IL-13 production in the MLN was not significantly altered in infected mice, although IL-13 expression by ILC2s was slightly increased (figure 3.1B).

In the blood, the proportions of IL-4 producing macrophages and T cells were increased in $H$. polygyrus-infected mice compared to naïve (figure 3.1C). IL-13 production was also increased in macrophages of infected mice. This difference, however, was not significant (figure 3.1D).

In the ear skin tissue, IL-4 production was slightly increased in eosinophils (although not significantly), and significantly increased in T cells in $H$. polygyrusinfected mice compared to their naive counterparts (figure 3.1E). The proportion of cells in the skin producing IL-13 was not significantly altered by $H$. polygyrus infection, however there was a trend for increased IL-13 production by ILC2s (figure 3.1F). 
Finally, IL-4 production in lung tissue was significantly increased in B cells and T cells, however this difference was not biologically significant in B cells as the difference was very small (0.004\% in uninfected versus $0.022 \%$ in infected mice) (figure $3.1 G$ ). IL-13 production in the lung was slightly increased in T cells, although not significantly (figure 3.1H). 


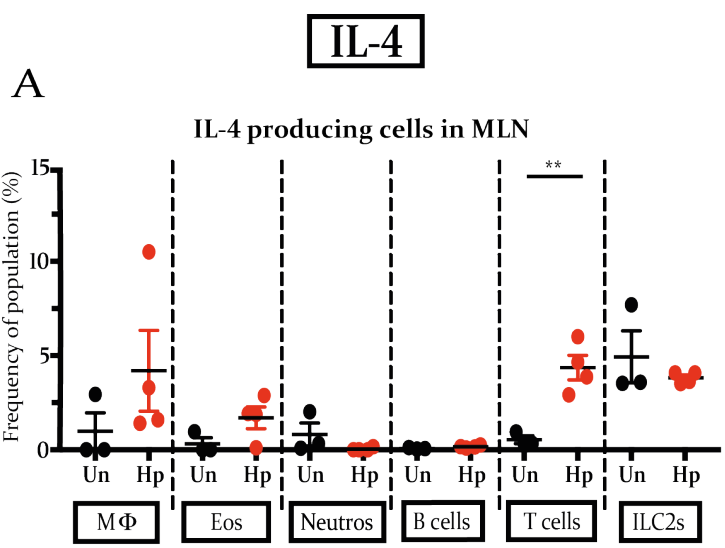

C

IL-4 producing cells in blood

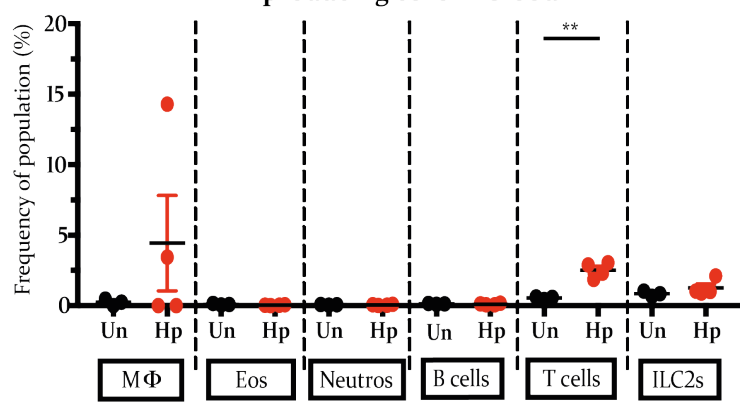

$\mathrm{E}$

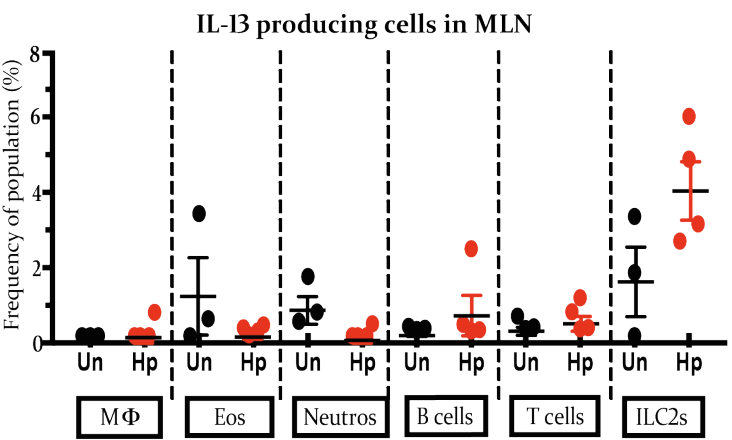

$\mathrm{D}$

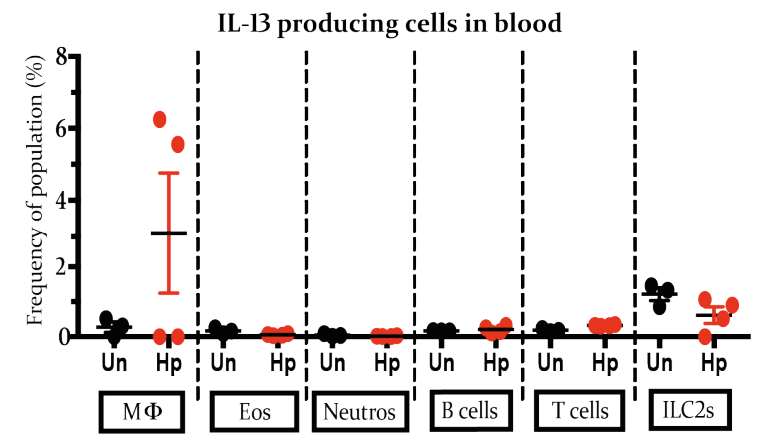

$\mathrm{F}$
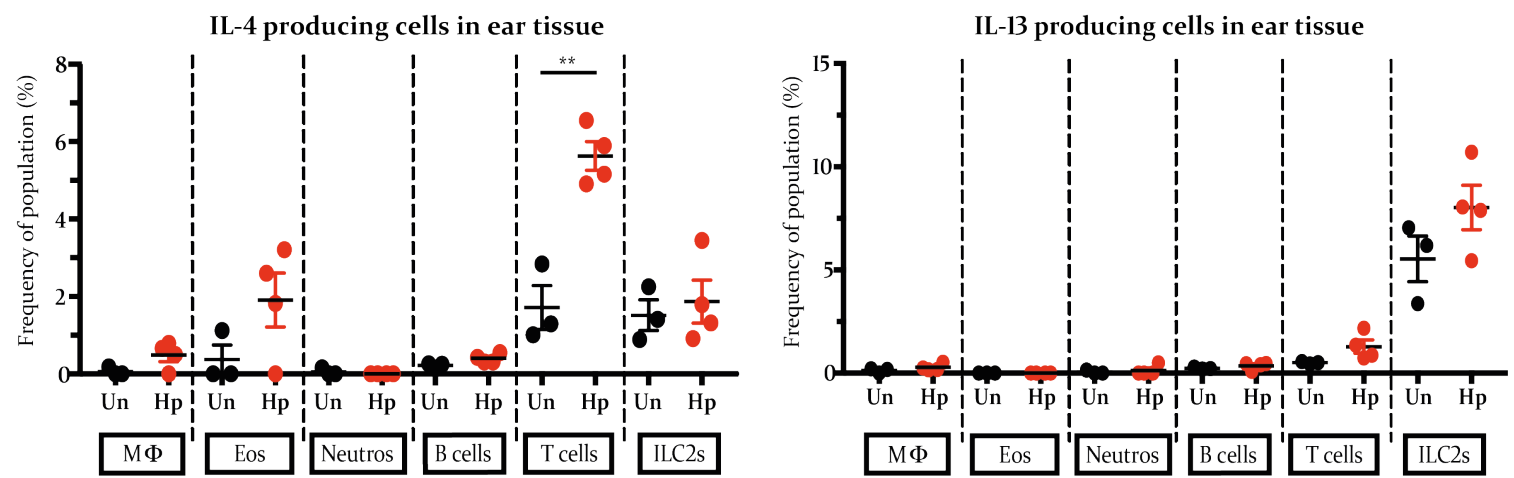

G

$\mathrm{H}$
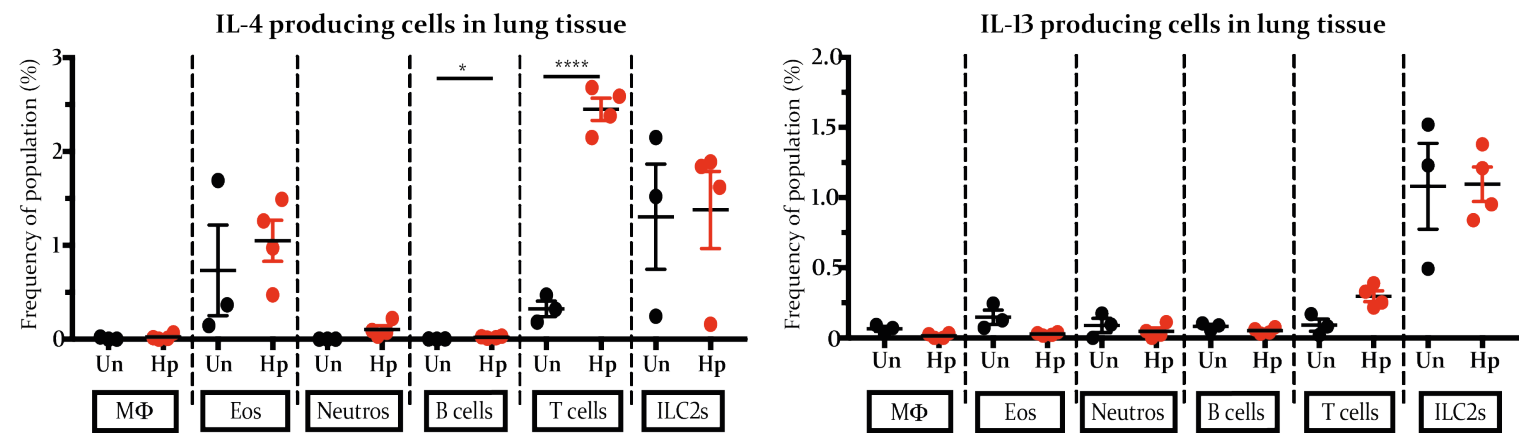

Figure 3.1: The effects of $H$. polygyrus infection on systemic Th2 responses.

Isolated cells from various tissues (MLN, whole blood, ear tissue and lung tissue) from uninfected mice (black) or $H$. polygyrus infected mice (red) analyzed by flow cytometry. IL-4 (A, C, E, G) and IL13 (B, D, F, H) cytokine expression were assessed in various immune cells (macrophages, eosinophils, neutrophils, B cells, T cells, ILC2s). Y-axis depicts frequency of IL-4 or IL-13 producing cells within each population. Graphs show mean \pm SEM, statistical significance was determined with a Student's unpaired T-test. ${ }^{*} \mathrm{p}<0.05,{ }^{* *} \mathrm{p}<0.01,{ }^{* * *} \mathrm{p}<0.001,{ }^{* * *} \mathrm{p}<0.0001$. MLN - mesenteric lymph nodes, $\mathrm{M} \phi$ - Macrophage, Eos - eosinophils, Neutros - neutrophils. 


\subsection{Discussion}

Previous studies found that $H$. polygyrus induces an $\mathrm{IL}-4^{+}$Th2 cell population in various organs, including blood, spleen, and lung. ${ }^{179}$ The results above coincide with these previous studies and supplement them by demonstrating this population of IL-4 producing $\mathrm{T}$ cells is also present in ear skin tissue in $H$. polygyrus-infected mice. IL-4 has been shown to alter skin homeostasis, but is not by itself able to induce inflammation or an AD phenotype. ${ }^{180}$ Furthermore, IL-4 induces AAMs, which have anti-inflammatory properties and are important for wound healing mechanisms. ${ }^{44,110-112}$ While the innate arm of the immune system is enough to induce AAMs in various tissues including the lungs and the skin, the adaptive arm

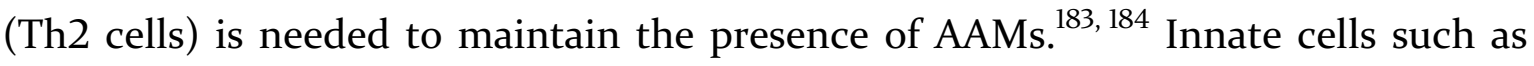
eosinophils were found to produce IL-4, although this increase in $H$. polygyrusinfected mice was not statistically significant above naive levels, and a larger sample size would be needed to determine if this difference was significant. The main source of IL-4 production in the skin of $H$. polygyrus-infected mice seems to be from $\mathrm{T}$ cells, making it likely that the stimulus for both induction and maintenance of AAMs is present in the skin of infected mice.

One of the immunomodulatory effects of $H$. polygyrus is the induction of AAMs, cells which can suppress Thl/Thl7-mediated responses and Th2 cell function and induce wound healing mechanisms. ${ }^{44,85}$ If these AAMs are indeed induced in the skin of $H$. polygyrus-infected mice, they could lead to suppression of skin inflammation and atopy, and to faster wound healing. Further study into AAMs in the setting of skin atopy would therefore be beneficial.

While IL-13 is mainly known for its pro-inflammatory and anti-parasitic properties, earlier this year a study indicated that IL-13 is important for maintaining homeostasis of the skin barrier. ${ }^{181}$ IL-13 is constitutively expressed by ILC2s in the skin, which coincides with the results above in uninfected mice. Infected mice seemed to have slightly elevated levels of IL-13 expressed by ILC2s, although this difference was not statistically significant. A larger sample size would be needed to determine if this difference is significant. 


\subsection{Conclusions}

Key points:

1) IL-4 is induced by $H$. polygyrus in various organs, including the MLN, blood, ear skin tissue, and lung tissue, and the main source of this IL- 4 is CD4 ${ }^{+} \mathrm{T}$ cells.

2) Innate cells such as eosinophils also produce IL-4, although there was no recorded difference in $H$. polygyrus-infected mice.

3) IL-13 is constitutively produced by ILC2s in the skin of mice, yet was not significantly altered by $H$. polygyrus infection. 
Chapter 4. H. polygyrus suppresses house dust mite-induced Th2 inflammatory responses in the ear 


\subsection{Introduction}

HDM is one of the most common environmental allergens causing airway inflammation in humans and is said to affect at least $15-20 \%$ of the population. ${ }^{185,186}$ The most common species of HDM are Dermatophagoides pteronyssinus and $D$. farinae and more than 20 allergens that induced specific IgE antibodies have been identified from these two species (named Der $\mathrm{p}$ or Der $\mathrm{f}$ followed by a number). ${ }^{187}$

\subsubsection{HDM contains a variety of allergens that induce allergic responses}

HDM allergens are divided into four families: proteases, non-proteolytic enzymes, lipid-binding proteins, and non-enzymatic components. ${ }^{186}$ Many allergens have enzymatic properties, e.g. proteases (Der p 1, 3, 6, and 9) are able to cleave tight junctions, weakening the epithelial barrier and contributing to sensitization of allergic responses. ${ }^{188}$

Mites are also contaminated with microbial pathogen-associated molecular patterns (PAMPs) such as lipopolysaccharides (LPS) and $\beta$-glucan that act as adjuvants in the priming of an immune response. ${ }^{189}$ These mite allergens and microbial PAMPs stimulate TLRs, C-type lectin receptors (CLR) and NOD-like receptors (NLR) on immune cells and epithelial cells and induce Th2-mediated inflammation. ${ }^{190-193}$ LPS through TLR4 signalling, was found to be a determining factor in the initiation of an immune response: low doses of LPS initiated Th2 responses with higher eosinophil recruitment, while high doses of LPS initiated stronger Thl responses with higher neutrophil infiltration. ${ }^{194}$

\subsubsection{HDM induces Th2 inflammation in the lungs}

HDM is often used in an experimental setting in mice to induce allergen-induced lung inflammation and airway hyper-responsiveness, mimicking asthma, as humans with asthma are often also allergic to HDM. ${ }^{195}$ This inflammation is characterized by increases in Th2 cytokines, innate cells including DCs, ILC2s, eosinophils, neutrophils, AAMs and basophils, and adaptive cells such as Th2 cells, and IgE producing B cells. $^{186}$ 
Inhalation of HDM in a murine model leads to TLR4-dependent recruitment of basophils, eosinophils and DCs to the lung draining LNs. It was found DCs are required for the initiation of the Th2 response, and basophils and eosinophils are the main innate sources of IL-4 for the induction of inflammation. ${ }^{196}$ HDM induces damage to the airway epithelial cells, causing release of alarmins such as IL-33, which contribute to the initiation of innate cell infiltration and activation such as ILC2s. ${ }^{74,186,190}$ Mice deficient in ST2 exhibit reduced airway inflammation, demonstrating the importance of innate cells and stimuli in HDM-induced airway hyperresponsiveness. ${ }^{197}$

HDM-induced lung inflammation leads to differentiation of Th2 cells. ${ }^{190}$ In the lymph nodes, it was found T follicular helper cells (Tfh) are an important source of IL-4, and induce class switching of B cells to IgG1 and IgE. ${ }^{198}$ In the lung tissue, Th2 cells were an important source of Th2 cytokines IL-4 and IL-13, mediating Th2 inflammation. ${ }^{198,199}$ B cells also migrate to the lung tissue, locally releasing $\operatorname{IgE}$ antibodies, which activate mast cells. ${ }^{198}$

\subsubsection{H. polygyrus suppresses HDM-induced Th2 inflammation in the lung}

H. polygyrus was found to suppress Der p 1-induced inflammation in the lung, with decreased infiltration of immune cells including eosinophils in the lung and decreased IL-5 and IL-13 production.. ${ }^{13}$, 123 This suppression of inflammation coincided with increased numbers of Tregs present in the lung and elevated levels of IL-10 and TGF $\beta .^{13}$ Furthermore, this protection was ablated when Tregs were depleted, and adoptive transfer of Tregs from $H$. polygyrus-infected mice to uninfected mice also conferred protection. ${ }^{13}$

Another study showed an expansion of a suppressive $C D 23^{\text {hi }}$ B cell population in the MLN of $H$. polygyrus infected mice. ${ }^{14}$ After adoptive transfer to uninfected mice, these cells were able to suppress Der p 1-induced asthma in an IL-10 independent manner, with decreased lung eosinophilia, airway pathology and IL-5 production. ${ }^{14}$

Together, these results indicate $H$. polygyrus-induced regulatory mechanisms are able to suppress murine lung inflammation models induced by HDM allergens. 


\subsubsection{The use of HDM in skin inflammation models}

HDM has been used in a murine model to induce strong Th2-mediated skin inflammation by ID injection in the ear skin tissue. ${ }^{200}$ This inflammation is characterized by an increase in $\mathrm{IL}-4^{+} \mathrm{CD} 4^{+} \mathrm{T}$ cells in the auricular LNs, which peaked between day 5-8, and production of HDM-specific IgGl antibodies from B cells. ${ }^{200}$ Furthermore, innate cells including eosinophils, basophils and mast cells increased in the ear skin tissue following HDM injection. ${ }^{200}$

HDM allergens have also been implicated in the development of AD, and the major mite protease allergens Der p 1 and Der $\mathrm{f} 1$ have been found on human skin. ${ }^{201}$ HDM protease allergens present on the skin tissue were seen to weaken the skin barrier, stimulate keratinocytes, and diminish epidermal barrier recovery in humans and mice. $^{202,203}$

These results demonstrate HDM-induced Th2 inflammation and impaired skin barrier function by topical application of mite allergens mimic components of AD. 


\subsection{Aims}

For this section, an ID injection of HDM was used to induce Th2 inflammation in the skin. ${ }^{200}$ There is limited information available on the immune cells that are activated during this HDM-induced inflammation in the ear skin tissue, and more research is needed.

Furthermore, $H$. polygyrus has been shown to suppress HDM-induced lung inflammation, however, it is currently not known if it is also able to suppress HDMinduced skin inflammation in the ear skin tissue. Therefore, more research is needed to determine the suppressive effects of $H$. polygyrus on ear skin tissue, using HDM to induce strong Th2 inflammation.

Specific goals:

1) To determine what immune cells are present in the ear draining $\mathrm{LN}$ and ear skin tissue during HDM-induced inflammation, and to investigate if this changes over time.

2) Investigate if $H$. polygyrus can suppress HDM-induced Th2 inflammation in the auricular LNs by inducing Tregs.

3) To determine if $H$. polygyrus can suppress HDM-induced Th2 inflammation in the ear skin tissue by inducing Tregs.

4) To determine if a 28-day $H$. polygyrus infection is more suppressive in the ear skin tissue than a 14-day $H$. polygyrus infection. 


\subsection{Results}

\subsubsection{ID injection of HDM in the ear induces substantial Th2 inflammation}

The previous chapter revealed there was an induction of $\mathrm{IL}-4^{+} \mathrm{T}$ cells in the ear skin tissue after infection with $H$. polygyrus. To determine the effect IL-4 could have on HDM-induced Th2 inflammation, it is important to know what cells will be present over time, especially during the peak of inflammation.

For this experiment, mice were injected ID with whole HDM at different time points ( 3 days, 6 days and 9 days) before the endpoint at day 0 (figure 4.1A). Both the auricular LNs and one ear from each mouse was taken for analysis by flow cytometry. CD $45^{+}$immune cells isolated from the LN (supplementary figure 1) were analysed for the presence of ILC2s (lin ${ }^{-} \mathrm{CD} 4^{-} \mathrm{CD}^{+} \mathrm{K}^{+} \mathrm{KLRG}^{+} \mathrm{ICOS}^{+} \mathrm{ST}^{+}$), T cells $\left(\mathrm{CD}^{+} \mathrm{CD}^{+}\right)$, and $\mathrm{ST} 2^{+} \mathrm{T}$ cells as a marker for IL-33 responsive Th2 cells. Lineage markers for negatively gating on ILC2s were: B220, CD8, CD1lb, CD1lc, FceRI, and NK1.1. From the ear skin tissue, $\mathrm{CD} 45^{+}$immune cells (supplementary figure 1) were stained for the presence of innate cells including eosinophils $\left(\mathrm{CD} \mathrm{b}^{+}\right.$Siglec $\mathrm{F}^{+}$), neutrophils $\left(\mathrm{CDIlb}^{+} \mathrm{Ly}_{6 \mathrm{G}^{+}}\right)$, basophils $\left(\mathrm{FccRI}^{+} \mathrm{DX}^{+}\right.$c-kit $\left.{ }^{-}\right)$and mast cells $\left(\mathrm{FcERI}^{+} \mathrm{c}-\right.$ $\left.\mathrm{kit}^{+} \mathrm{DX} 5^{-}\right)$.

Previous studies showed an increase in eosinophils, basophils and mast cells in the ear skin tissue, and IL- $4^{+} \mathrm{CD} 4^{+} \mathrm{T}$ cells in the auricular LNs peaking between day 5$8 .^{200}$ In the auricular LN, there was an increase in total live immune cells after HDM injection, which peaked between day 6 and day 9 (figure 4.1B). There were no ILC2s detected in the LN (data not shown), and total $\mathrm{CD}^{+} \mathrm{T}$ cells increased in number over time (figure 4.1C). The numbers of $\mathrm{CD}^{+} \mathrm{T}$ cells expressing ST2 increased slightly over time, however this difference was not significant (figure 4.1D).

In the ear skin tissue, there was an increase in total live immune cells, which peaked at day 6 post-HDM injection (figure 4.1E). The eosinophil population was apparent in all samples, but peaked at day 9 (figure 4.1F). On the other hand, neutrophils made up 50\% of immune cells present in the ear skin tissue at day 3 and 6 , and dropped back to just above naive baseline at day 9 (figure 4.1G). There were no clear populations of basophils or mast cells present in the skin, nor did these populations 
appear to increase during HDM-induced inflammation (data not shown). These results supplement data from Camberis et al. ${ }^{200}$ demonstrating the presence of neutrophils early on in the immune response after HDM injection as well as eosinophils, and $\mathrm{T}$ cell expansion during the Th2 response.

\subsubsection{H. polygyrus suppresses HDM-induced Th2 inflammation in the skin, coinciding with a slightly increased proportion of Tregs in the LN}

Next, to determine if H. polygyrus is able to suppress HDM-induced inflammation, C57BL/6 mice were infected with 200 L3 larvae via oral gavage at day -14, injected ID with HDM on day 0 , and analysed on day 3, 7, and 10 (figure 4.2A). Both the auricular LNs and one ear from each mouse was taken for analysis by flow cytometry. $\mathrm{CD} 5^{+}$immune cells isolated from the LNs (supplementary figure 1) were analysed for the presence of $\mathrm{B}$ cells $\left(\mathrm{B}_{2} 2 \mathrm{O}^{+}\right)$, $\mathrm{T}$ cells $\left(\mathrm{CD}^{+} \mathrm{CD}^{+}\right)$, and Tregs $\left(\mathrm{CD}^{+}\right.$ $\mathrm{CD}^{+} \mathrm{FoxP}^{+}$). Furthermore, serum was extracted from whole blood to determine the levels of HDM-specific antibodies through ELISA.

First, in the LN, the total number of live immune cells present in HDM-only treated mice peaks at day 7 , confirming the results in section 4.3 .1 above (figure 4.2B) $\mathrm{H}$. polygyrus infection alone did not result in a change in cell number in the auricular LNs compared to naive levels. At day 7, the number of total immune cells in the LNs of infected mice after HDM injection is significantly decreased (threefold difference) in comparison to uninfected mice (figure 4.2B). As the peak of inflammation was at day 7 , and there was a significant difference between uninfected and infected mice after HDM injection, further analysis has focused on data from this time point.

Proportions of B cells in the auricular LNs did not differ in proportion between uninfected or infected mice after HDM injection (figure 4.2C). However, B cell numbers and HDM-specific antibodies are decreased (although not significantly) in H. polygyrus-infected mice (figure 4.2C, D). 
There was no difference in $\mathrm{T}$ cell proportions between infected and uninfected mice with HDM injection, although $\mathrm{T}$ cells numbers did seem to be decreased (figure 4.2E).

Finally, as expected Treg proportions were increased in all $H$. polygyrus-infected mice in comparison to uninfected mice (figure 4.2F), however the actual number of Tregs is still decreased in H. polygyrus-infected mice with HDM injection, reflecting the overall size of the auricular LNs. These results suggest there is an overall suppression of immune cells present in the auricular LNs by $H$. polygyrus infection, which coincides with an increase in Treg proportions in the LNs.

\subsubsection{H. polygyrus suppresses HDM-induced Th2 inflammation in the skin, coinciding with a slightly increased proportion of Tregs in the ear skin tissue}

$\mathrm{CD}^{+} 5^{+}$immune cells isolated from ear skin tissue (supplementary figure 1) were analysed for the presence of $\mathrm{T}$ cells $\left(\mathrm{CD}^{+} \mathrm{CD}^{+}\right)$, Tregs $\left(\mathrm{CD}^{+} \mathrm{CD}^{+} \mathrm{FoxP}^{+}\right)$, eosinophils $\left(\mathrm{CDllb}^{+}\right.$Siglec $\left.\mathrm{F}^{+}\right)$, and AAMs $\left(\mathrm{CDllb}^{+} \mathrm{CD}^{+} 4^{+}\right.$Relm $\left.\alpha^{\text {hi }}\right)$. Using the same experimental protocol (figure 4.3A) as for the auricular LNs, in the ear skin tissue there was no significant difference in total live immune cells between groups and even over time (figure 4.3B). There was a slight difference on day 7, where uninfected mice after HDM injection had the highest number of immune cells, which coincides with data from the auricular LNs. This points to day 7 as the peak of the inflammation after ID injection of HDM, and further analysis in this section will focus on data from this time point.

In both groups containing infected mice, $\mathrm{CD}^{+} \mathrm{T}$ cells proportions were increased, although this difference was not significant, and absolute numbers did not differ (figure 4.3C). Proportions of FoxP3 ${ }^{+}$Tregs were also increased in both groups of infected mice, suggesting the increase of $\mathrm{CD}^{+} \mathrm{T}$ cells is most likely due to the increased presence of Tregs in the skin (figure 4.3D). 
Eosinophils were recruited to the ear skin tissue after HDM injection, and although not significant, there is a trend towards a lower proportion of eosinophils present in the ear skin tissue in infected mice (figure 4.3E).

Finally, infected mice without HDM injection showed an increase in AAM population in the ear skin tissue. Both groups of mice injected with HDM (uninfected and infected) showed an increase in this AAM population in comparison to naive mice, however there was no significant difference between these two groups (figure 4.3F).

\subsubsection{Chronic infection with $H$. polygyrus suppresses HDM-induced Th2 inflammation further}

H. polygyrus can establish a chronic infection within several strains of mice. ${ }^{92}$ Treg numbers peak at 28 days post-infection in the gut-draining LNs and have a strong regulatory phenotype. ${ }^{16}$ Therefore, a 28-day infection with $H$. polygyrus was used to determine if there was a stronger suppression of HDM-induced skin inflammation in the ear tissue at 28 days, compared to the 14-day model.

For this section, 4C13R mice were used to determine the expression of the Th2 cytokines IL-4 and IL-13. Mice were infected with 200 L3 larvae at day -28, and injected ID with HDM at day 0 . The mice were sacrificed at day 7 , and the auricular LNs were taken for analysis by flow cytometry (figure $4.4 \mathrm{~A}$ ).

Coinciding with previous data from the 14-day model (figure 4.2), total numbers of live immune cells present in the auricular LNs are increased in uninfected mice after HDM injection, and $H$. polygyrus infection significantly reduces these numbers (figure 4.4B).

Previous data showed a decreased absolute number of B cells in infected mice after HDM injection. After 28-day infection with $H$. polygyrus, there is a significant reduction not only in B cell number, but also in B cell proportion (figure $4.4 \mathrm{C}$ ), 
suggesting a 28-day model of $H$. polygyrus infection is more suppressive than a 14 day model.

Finally, $\mathrm{CD} 4^{+} \mathrm{T}$ cell numbers are significantly reduced in $H$. polygyrus-infected mice after HDM injection in comparison to uninfected mice after HDM injection (figure 4.4D). Of these $\mathrm{CD} 4^{+} \mathrm{T}$ cells, there is an increased proportion and number of $\mathrm{IL}-4^{+}$ $\mathrm{T}$ cells in uninfected mice with HDM injection compared to naive mice, and this is decreased in the $H$. polygyrus-infected group (figure 4.4D). 
A

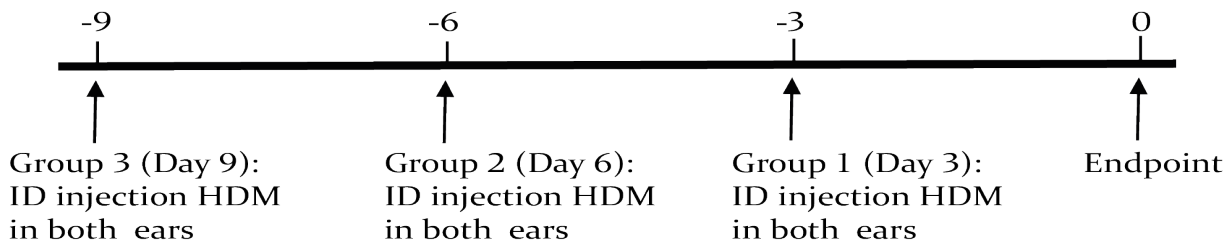

B

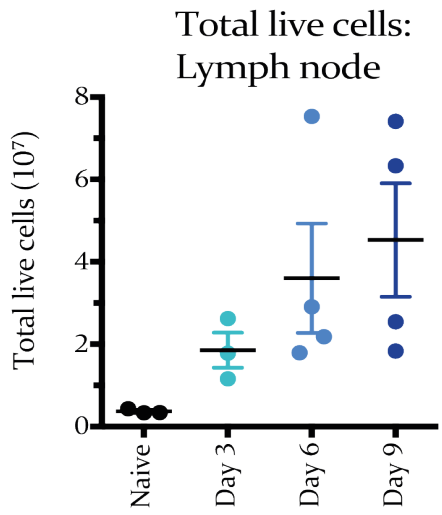

$\mathrm{D}$

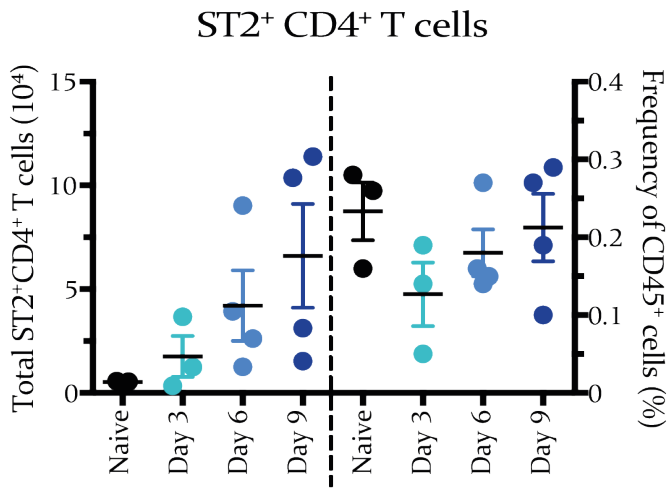

F

Eosinophils

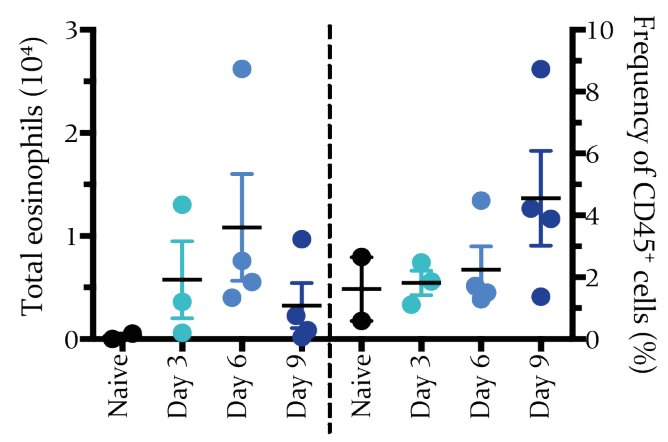

C

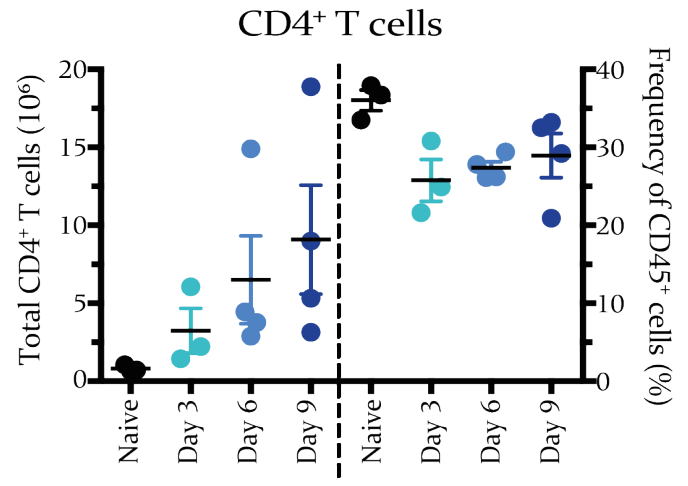

E

Total live cells:

Ear tissue

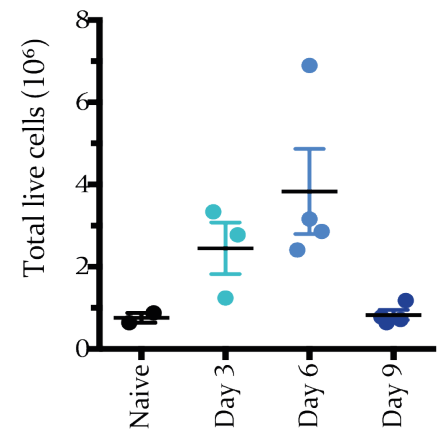

G

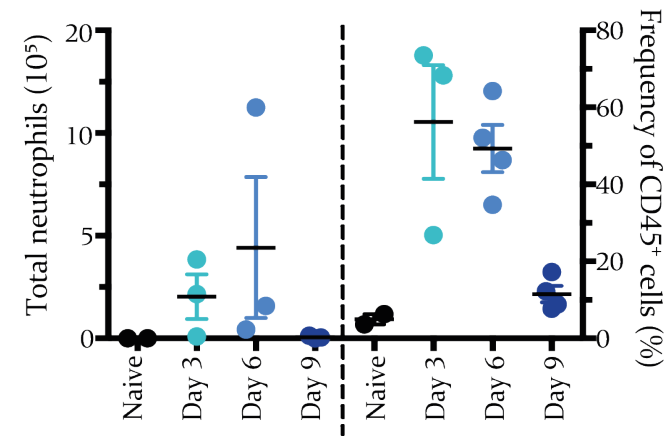

Figure 4.1: Time course of ID injected HDM-induced Th2 inflammation.

Cells from LN and ear skin tissue were stained and analysed by flow cytometry A) Timeline of the experiment. B) Total live cells, C) CD4 ${ }^{+} \mathrm{T}$ cells, and D) $\mathrm{ST} 2^{+} \mathrm{CD} 4^{+} \mathrm{T}$ cells present in the auricular $\mathrm{LNs}$ of mice in naive and HDM injected mice during different time points over the time course. E) Total live cells, F) eosinophils, and D) neutrophils present in the ear skin tissue of mice in naive and HDM injected mice during different time points over the time course. Graphs show mean \pm SEM. 
A

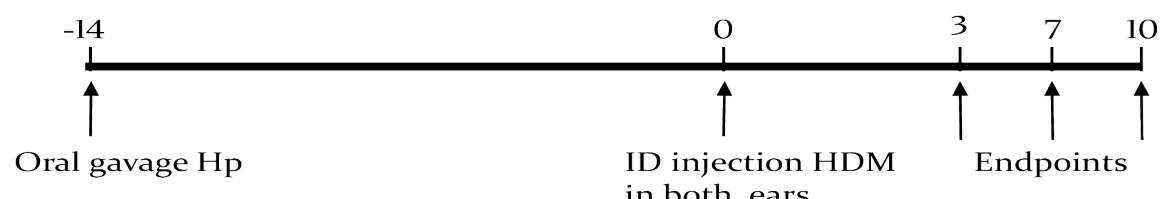

B

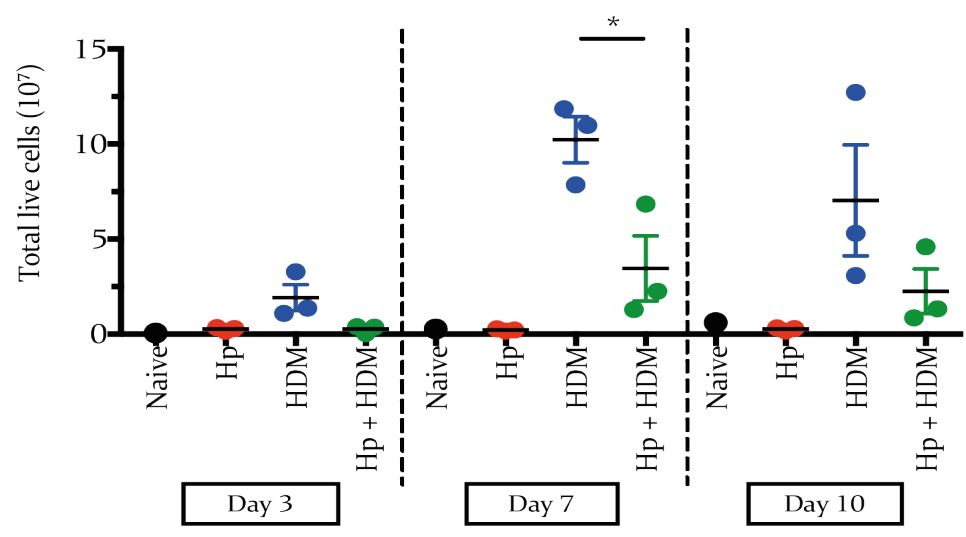

C

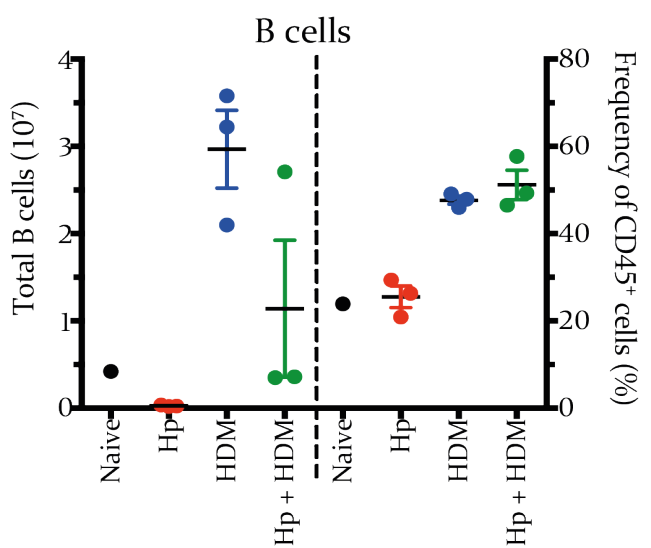

$\mathrm{E}$

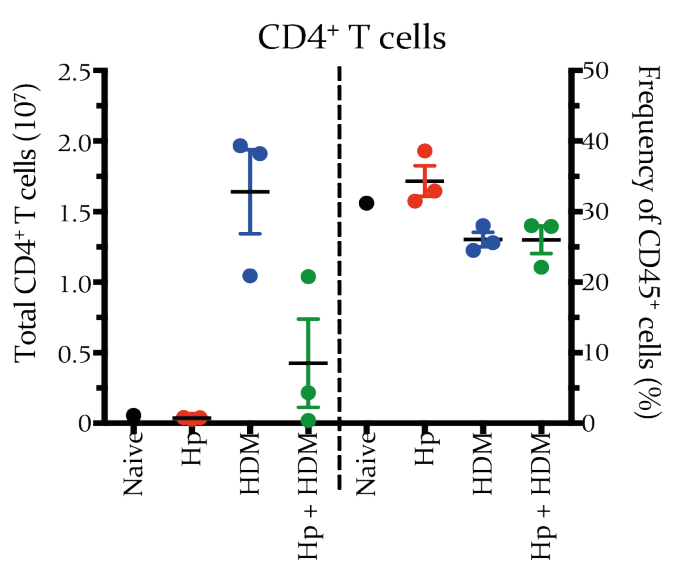

$\mathrm{D}$

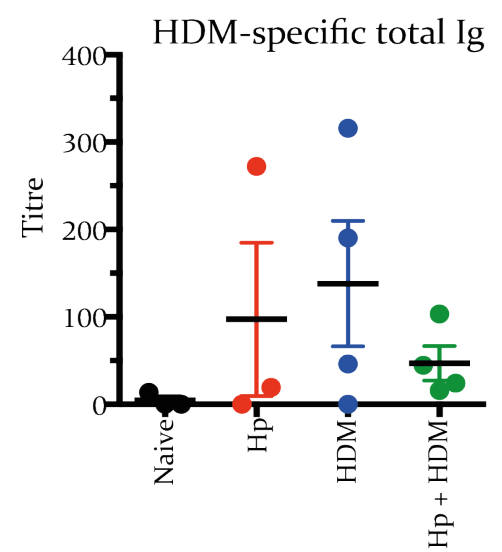

$\mathrm{F}$

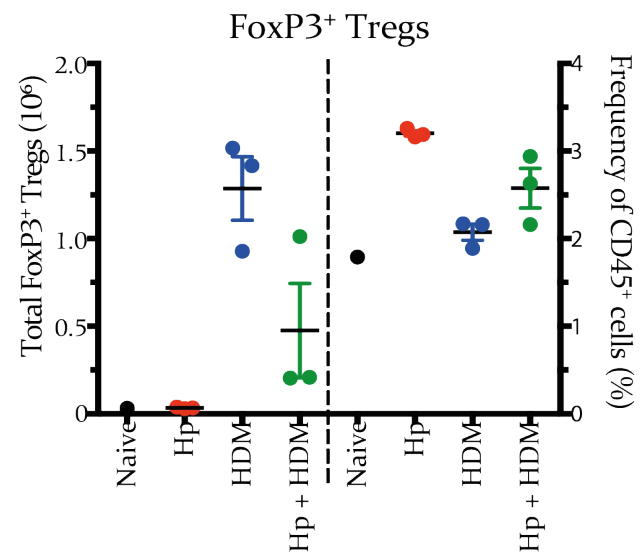

Figure 4.2: $H$. polygyrus suppresses HDM-induced inflammation in the auricular LN and induces Tregs.

A) Model for $H$. polygyrus infection and ID injection of HDM (numbers depict the day) B) Total live cells present in the auricular LNs at day 3, 7 and 10. Flow cytometry analysis at day 7 in the LN C) total live B cells, E) total $\mathrm{CD}^{+} \mathrm{T}$ cells and F) $\mathrm{CD}^{+} \mathrm{FoxP}^{+}$Tregs. D) HDM-specific total Ig measured by ELISA in the serum. Titre is defined as the reciprocal serum dilution at which the dilution curve passes $50 \%$ of the maximum OD of the whole plate. Graphs show mean \pm SEM, statistical significance was determined with a Student's unpaired T-test. * $\mathrm{p}<0.05$ 
A

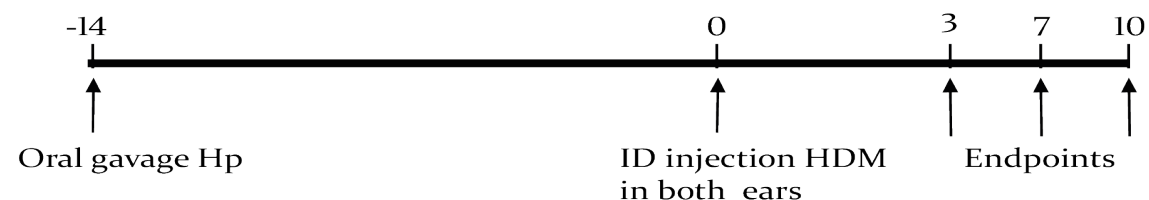

B

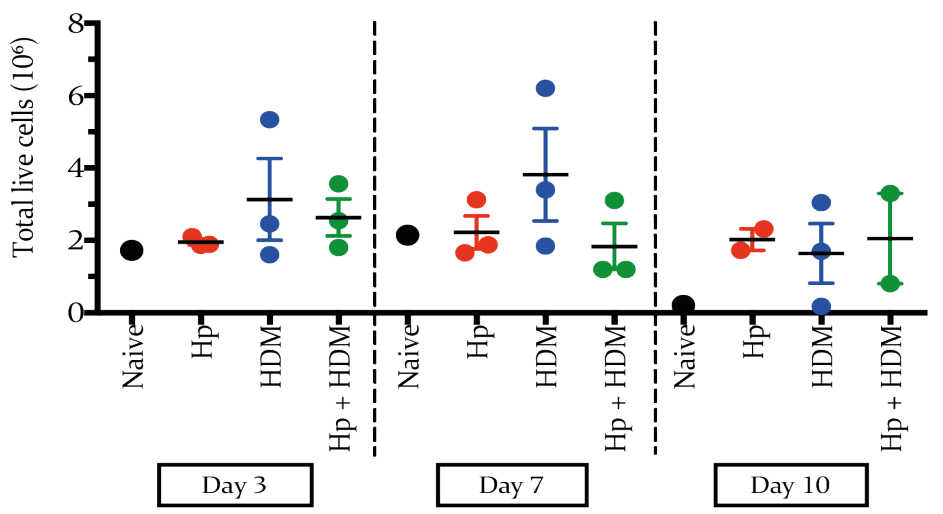

C

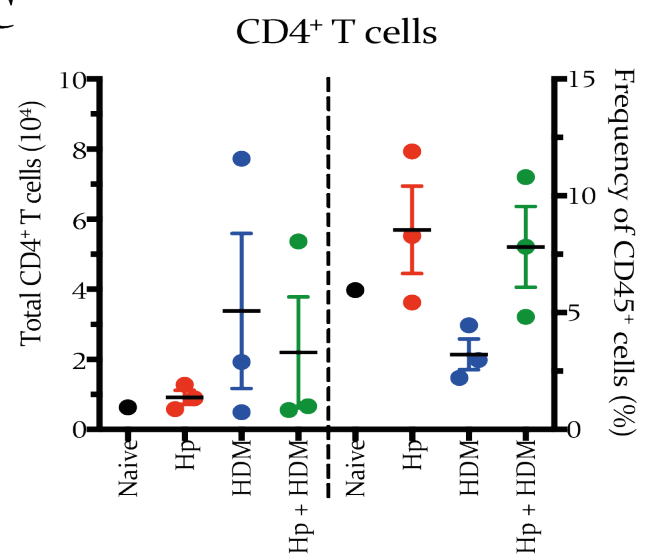

$\mathrm{E}$

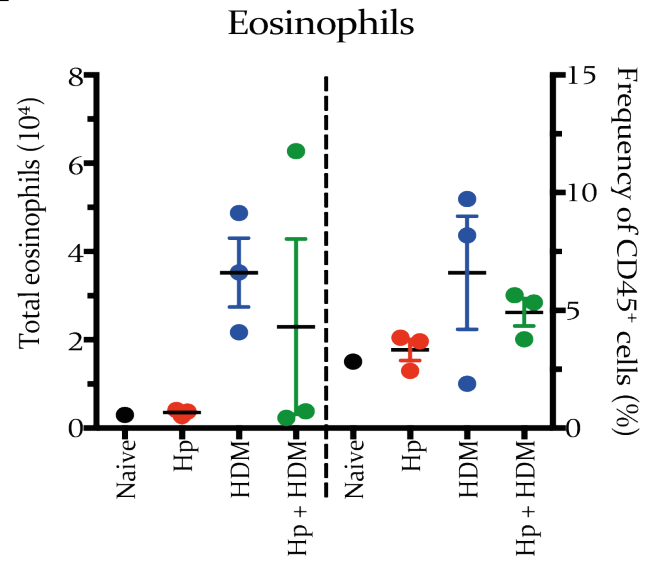

$\mathrm{D}$

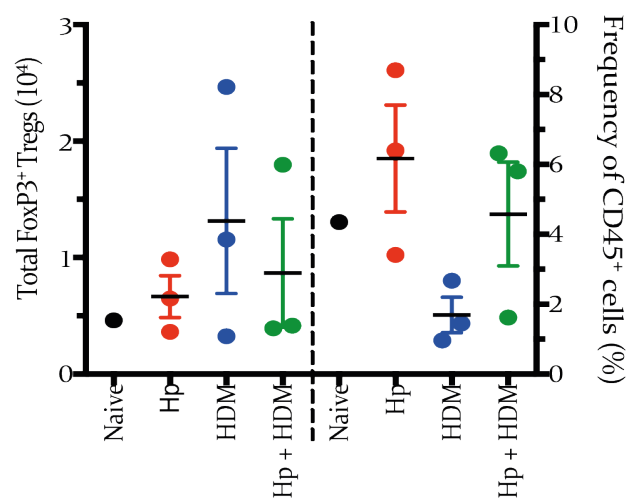

F

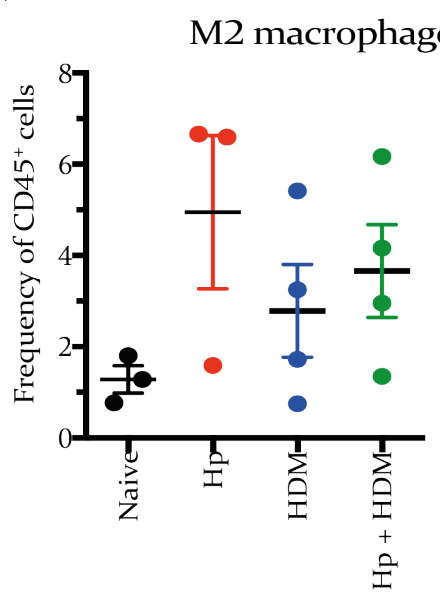

Figure 4.3: H. polygyrus affects HDM-induced inflammation in the ear skin tissue and induces Tregs and M2 macrophages/AAMs.

A) Model for $H$. polygyrus infection and ID injection of HDM (numbers depict the day) B) Total live cells present in the ear skin tissue at day 3, 7 and 10. Flow cytometry analysis at day 7 in the ear skin tissue C) $\mathrm{CD}^{+} \mathrm{T}$ cells, D) $\mathrm{CD}^{+}{ }^{+} \mathrm{FoxP}^{+}$Tregs, E) total live eosinophils, and F) total live M2 macrophages/AAMs. Graphs show mean \pm SEM 
A

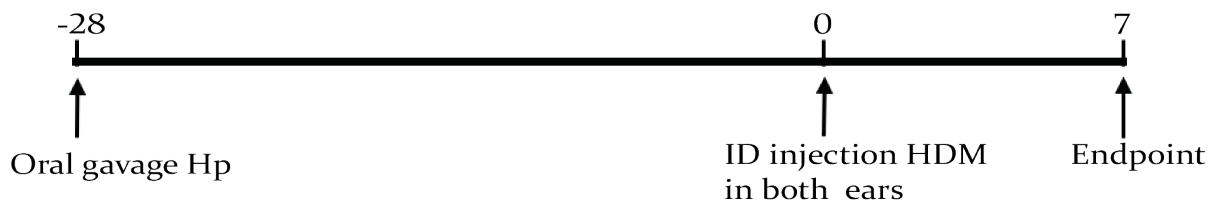

B

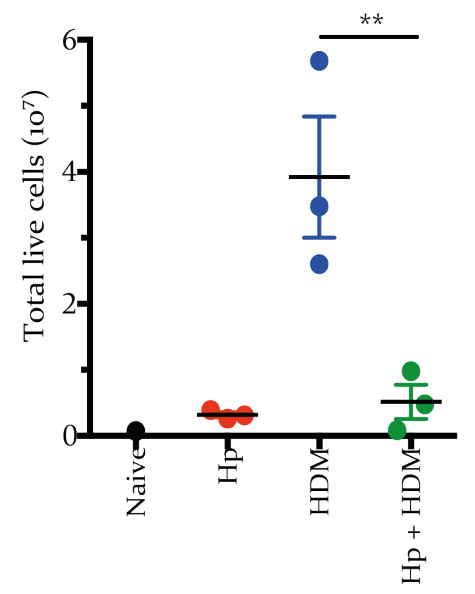

D

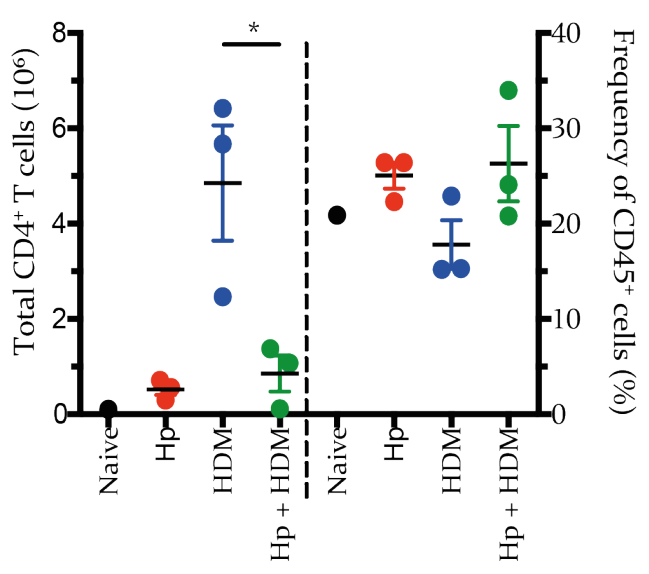

C

B cells

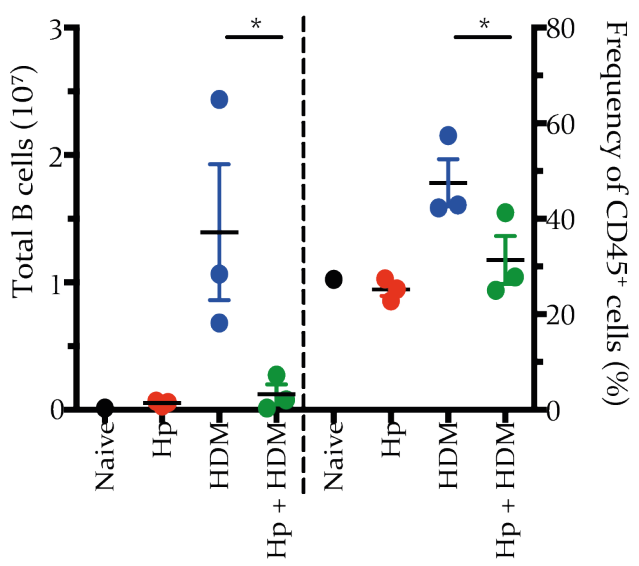

$\mathrm{AmCyan}^{+}(\mathrm{IL}-4) \mathrm{CD}^{+} \mathrm{T}$ cells

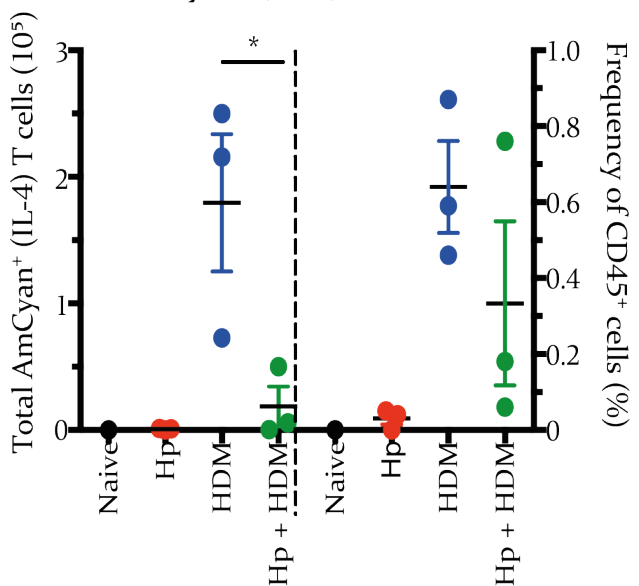

Figure 4.4: H. polygyrus suppresses HDM-induced inflammation in the auricular LNs during chronic infection.

A) Model for $H$. polygyrus infection and ID injection of HDM (numbers depict the day) B) Total live cells present in the auricular LNs. Flow cytometry analysis in the LN C) total live B cells, D) total $\mathrm{CD}^{+} \mathrm{T}$ cells and $\mathrm{AmCyan}^{+}(\mathrm{IL}-4) \mathrm{CD} 4+\mathrm{T}$ cells. Graphs show mean \pm SEM, statistical significance was determined with a Student's unpaired T-test. ${ }^{*} \mathrm{p}<0.05,{ }^{* *} \mathrm{p}<0.01$ 


\subsection{Discussion}

Previous studies have shown HDM induces strong inflammation in the lung, mainly through TLR-dependent mechanisms and cleaving of tight junctions of epithelial cells leading to secretion of alarmins such as IL-33. ${ }^{188,190,191}$ While there is limited data available on the initiation of a Th2 inflammatory response by ID injection of HDM, it has been shown to be sufficient in activating DCs without the presence of TLSP. $^{143}$

The results above demonstrate ID injection of HDM induces strong inflammation in the auricular LN and ear skin tissue as seen by the significant increase in total live immune cells during the peak of the inflammation on day 7. This inflammation is characterized by the presence of innate cells such as eosinophils and neutrophils in the skin and $\mathrm{CD} 4^{+}$Th2 cells in the LN.

Previous studies have also shown $H$. polygyrus was able to suppress HDM-induced lung inflammation through the induction of Tregs. ${ }^{13}$ Concurring with these studies, it was found $H$. polygyrus suppressed HDM-induced Th2 inflammation in the skin characterized by decreased numbers of immune cells present in the auricular LN and ear skin tissue. In the auricular LNs, a reduced number of B cells and CD4 ${ }^{+} \mathrm{T}$ cells were present, although a larger sample size would be needed to determine if this difference was significant. Coinciding with a lower number of B cells, $H$. polygyrus-infected mice had less HDM-specific antibodies present after HDM injection than uninfected mice. Interestingly, one infected mouse that did not receive an HDM injection had HDM-specific antibodies in the serum, suggesting there might be some cross-reactivity between mite allergens and parasitic antigens. This phenomenon has recently been demonstrated in a paper comparing the molecular structures of common allergens and parasitic antigens. ${ }^{204}$

In the ear skin tissue, there was no significant difference found in total immune cells present in the tissues after HDM injection or $H$. polygyrus infection. As expected for Th2-driven inflammation, eosinophils were recruited to the ear skin tissue after HDM injection, and there was a trend towards a lower number and proportion of eosinophils in the ear skin tissue of infected mice. Again, a larger 
sample size would be needed to determine the significance. However, there were issues with the digestion enzyme cocktails used in these experiments (primarily concerning CD4-cleavage from the surface of cells with some enzymes), and optimisation of digestion conditions was ongoing throughout the experimental time-frame.

H. polygyrus-infected mice had an increased proportion of Tregs in the auricular LNs, which coincides with previous studies by Wilson et al. ${ }^{13}$ and is most likely a key mechanism to the suppression of inflammation in the ear skin tissue. Furthermore, H. polygyrus infection caused an increase in $\mathrm{T}$ cells in the ear skin tissue with a larger proportion of these being FoxP3 ${ }^{+}$Tregs. While Tregs are evident in the skin in these experiments, they are absent in the skin of patients with $\mathrm{AD} .{ }^{205}$ This suggests Tregs present in the ear skin tissue after HDM-injection are likely induced by $H$. polygyrus infection. Further studies using depletion of Tregs in $H$. polygyrusinfected mice, and transfer of Tregs from $H$. polygyrus-infected mice to uninfected mice, would demonstrate the importance of Tregs in the suppression by $H$. polygyrus.

Finally, in the previous chapter it was hypothesized that the release of IL-4 in the skin of infected mice could lead to induction of AAMs. AAMs are one of the key regulatory cell types induced by $H$. polygyrus. ${ }^{113} \mathrm{H}$. polygyrus-infected mice did seem to have an increased proportion of AAMs present in the ear skin tissue, however after HDM injection, there did not seem to be a difference between uninfected and infected mice. These results suggest AAMs are also induced after HDM-injection, and suppression of HDM-induced skin inflammation by $H$. polygyrus is not dependent on AAMs. Depletion of AAMs (using clodronate-coated liposomes), blocking of their effector molecules (such as arginase-1), or use of mice in which depletion of AAMs is possible (LysMCre or CD1lb-DTR), may shed more light onto the role of AAMs in this model.

After more chronic infection with $H$. polygyrus, Tregs peak at day 28 and have a strong modulatory profile, characterized by CD103 expression, antigen-specific IL-10 production and increased TGF $\beta$ on the cell surface of CD4 ${ }^{+}$T cells. ${ }^{16}$ Using a 28 -day chronic infection model with $H$. polygyrus, total number of live immune cells in the 
auricular LNs were decreased in comparison to uninfected mice after HDM injection. The 28-day model was more suppressive than the 14-day model, as $H$. polygyrus infection suppressed expansion of B cell and IL- $4^{+} \mathrm{T}$ cell proportion as well as absolute numbers. These IL- $4^{+} \mathrm{T}$ cells are most likely Tfh cells, as they have been shown to be important for the class switching of B cells, which would coincide with a lower HDM-specific antibody production in the serum and decreased proportion of B cells in the LN. ${ }^{198}$ While immune cells in the auricular LNs seem to be more suppressed with a 28-day infection before HDM injection, a 14-day infection is sufficient for $H$. polygyrus to significantly suppress HDM-induced skin inflammation.

Future experiments, as mentioned before, would include determining the importance of Tregs in H. polygyrus-mediated suppression of HDM-induced Th2 inflammation in the ear skin tissue. Furthermore, due to issues with digestion enzyme cocktails, more research is needed to accurately determine the suppression of immune cells present in the ear skin tissue by $H$. polygyrus. Another enzyme cocktail could be used to study $\mathrm{T}$ cells and $\mathrm{FoxP}^{+}$Tregs in the ear skin tissue. Furthermore, culturing these Tregs in vitro with other lymphocytes would determine if Tregs induced by $H$. polygyrus distal to the gut are more suppressive than Tregs induced in uninfected mice. 


\subsection{Conclusions}

Keypoints:

1) ID HDM injection into the ear skin tissue induced strong Th2 inflammation characterized by Th2 cells, B cells, eosinophils and neutrophils.

2) H. polygyrus suppressed HDM-induced Th2 inflammation in the ear skin tissue and auricular LN, characterized by decreased number of immune cells present.

3) H. polygyrus infection increased the proportions of Tregs in LNs distal to the gut, i.e. the auricular LNs, and in the ear skin tissue. However, this difference was not significant.

4) H. polygyrus induced AAMs in the ear skin tissue under homeostatic conditions, and after HDM injection, there was no difference between uninfected and infected mice.

5) A chronic 28-day infection with $H$. polygyrus was more suppressive than a 14-day $H$. polygyrus infection, and resulted in a decreased number and proportions of immune cells such as B cells and IL-4 ${ }^{+} \mathrm{T}$ cells in the $\mathrm{LN}$. 
Chapter 5. H. polygyrus suppresses hapten-induced skin allergy 


\subsection{Introduction}

One of the most common forms of AD is CHS, which is mediated by $\mathrm{T}$ cells after activation by APCs such as DCs. This skin inflammation is characterized by dermal infiltration of immune cells such as Th2 cells, mast cells, eosinophils, increased IL-4 production, and increased IgE levels. ${ }^{161,206,207}$ Furthermore, as with AD patients, mice exhibited increased epidermal hyperplasia and a decreased expression of filaggrin and tight junction proteins, leading to impaired skin barrier function and increased TEWL, which was found to correlate with AD severity. ${ }^{161,206-209}$

In an experimental setting, CHS is often induced by topical application of chemical haptens, such as oxazolone, MC903 or FITC. Chemical haptens are small molecules, which induce an immune response after binding to soluble or cell-associated proteins. ${ }^{162}$ While most haptens induce an acute Thl response in the skin, multiple challenges to the skin over a longer period will induce a shift from a Thl response towards a Th2 immune response that is similar to AD in humans. ${ }^{161,207}$ This shift towards a Th2 response after repeated sensitization by chemical haptens, demonstrates the importance of environmental factors for the onset of $\mathrm{AD}$, and demonstrates hapten-induced skin inflammation can further amplify damage to the skin barrier. ${ }^{62,161}$

Application of FITC, in combination with DBP, although an acute model, induces Th2-dominated CHS with high levels of IL-4 and IL-10 seen in the skin even though it is an acute model. ${ }^{162,210}$ DBP is used as a solvent for FITC, and also acts as an adjuvant to enhance the potency of the hapten through induction of TLSP by keratinocytes. $^{211,} 212$ TSLP is important for the initiation of Th2-mediated inflammation via suppression of IL-12 production by DCs, expression of costimulatory molecules, and upregulation of chemokine ligands (CCL) 17 and CCL22. 55, 213, 214 Through these processes DCs are activated towards a Th2-activating phenotype. As elevated levels of TSLP produced by keratinocytes have also been seen in humans with $\mathrm{AD}, \mathrm{DBP}-\mathrm{FITC}$-induced CHS looks to be a suitable model to study $\mathrm{AD}$ in mice. ${ }^{213,215}$ 
Research using this model of AD to induce skin allergy has found specific subsets of dermal DCs, namely the CDllb ${ }^{+} \mathrm{DCs}$ and TN DCs, to be responsible for the activation of Th2 cells in the skin-draining LN. ${ }^{143}$ These TN DCs were highly responsive to TSLP, and not activated in the absence of this cytokine. ${ }^{143}$ Another study showed there was an increase in ear thickness, eosinophil infiltration, and IgE levels after DBP-FITC application to ear skin. ${ }^{215}$ Furthermore, anti-IL-4, anti-IL-5, and mast cell depletion reduced ear thickness, and major histocompatibility complex II (MHCII) KO mice and STAT6 KO mice were protected from DBP-FITCinduced CHS. ${ }^{215}$ These results demonstrate the importance of MHCII-dependent DC activation of $\mathrm{CD} 4{ }^{+} \mathrm{Th} 2$ cells to initiate Th2 inflammation, and that innate cells such as eosinophils and mast cells contribute to inflammation in the skin. ${ }^{215}$ 


\subsection{Aims}

The previous chapter demonstrated $H$. polygyrus was able to suppress HDMinduced skin inflammation, coinciding with an increase in Treg proportions in the auricular LNs and ear skin tissue. However, as TSLP is not detected in this model, and has been demonstrated to be elevated in humans with $\mathrm{AD}$, it is not entirely suitable as a model for AD. ${ }^{143,200,213}$ DBP-FITC has been shown to mimic the human CHS phenotype more accurately. ${ }^{213,215}$ As yet, it is not known if $H$. polygyrus is able to influence DBP-FITC-induced CHS. Therefore, for this section, a murine model of DBP-FITC-induced Th2 inflammation was used to study the effect of H. polygyrus on skin allergy.

Specific goals:

1) Determine if $H$. polygyrus is able to suppress DBP-FITC-induced CHS through measuring ear thickness as a read-out for inflammation, and TEWL as a measure for skin barrier function.

2) Determine if $H$. polygyrus is able to influence DC phenotype or numbers after DBP-FITC treatment on the ear skin tissue.

3) Determine if $H$. polygyrus is able to suppress $C D 4^{+} T$ cells subsets, and cytokine production of lymphocytes, by inducing Tregs in the auricular LNs.

4) Determine what cytokines are being produced in the ear skin tissue and if $H$. polygyrus is able to influence the production of these cytokines 


\subsection{Results}

\subsubsection{H. polygyrus suppresses skin inflammation in DBP-FITC-induced CHS}

While the previous chapter demonstrated HDM-induced skin inflammation was suppressed by $H$. polygyrus, further research is needed to determine if $H$. polygyrus is also suppressive in a more suitable model of $\mathrm{AD}$.

For this experiment, mice were infected with 200 L3 larvae via oral gavage at day 14 , the flanks of the mice were shaved at day -7 so no irritation from the shaving would remain during sensitization by DBP-FITC on the flanks (on day 0 and 2), and finally, mice were challenged with DBP-FITC on the left ear and the vehicle DBP alone on the right ear on day 6 (figure 5.1A). Before challenge and every day after challenge, ear thickness was measured to determine the level of inflammation, and TEWL was measured to determine if skin barrier function was compromised.

While the vehicle DBP alone did not increase ear thickness in mice, challenge with DBP-FITC increased the ear thickness of uninfected mice and the peak of inflammation was around day 1 and 2 after challenge (figure 5.2B). Infected mice challenged with DBP-FITC had significantly reduced ear thickness at day 2 after challenge (figure 5.2B). However, there was no difference in TEWL (figure 5.2C). These results suggest $H$. polygyrus is able to suppress DBP-FITC induced skin inflammation, but does not affect skin barrier function.

\subsubsection{H. polygyrus suppresses inflammation early in the immune response but does not alter DC phenotype in the skin}

DCs have been shown to be crucial for the initiation of DBP-FITC induced CHS. ${ }^{215}$ Furthermore, $H$. polygyrus infection induces a tolerogenic phenotype in intestinal $\mathrm{CD}_{11} \mathrm{c}^{\text {lo }}$ DCs and blocks the migration of CDllc ${ }^{\text {hi }}$ DCs to the MLN, but it is still not known if $H$. polygyrus infection influences DCs in other tissues, particularly the skin. ${ }^{128}$ Additionally, Tregs are able to influence the phenotype of DCs through CTLA-4 binding to CD86. ${ }^{100}$ 
Earlier results indicated HDM-induced inflammation seemed to be suppressed at day 3 in infected mice (figure 4.2B). Therefore, it was hypothesized Th2 inflammation in the skin could be suppressed through the alteration of DC phenotype or the blocking of migration to the auricular LNs.

For this experiment, mice were infected with 200 L3 larvae via oral gavage at day 14 , and treated once with DBP-FITC on the ear skin tissue of both ears on day 0 (figure 5.2A). At day 2, both LNs were harvested and processed according to protocol. Immune cells isolated from the LNs (supplementary figure 1) were stained and analysed using flow cytometry, and 5 DC subsets were surveyed for the cell numbers, frequency of cells that were FITC $^{+}$(i.e. DCs that had taken up the treatment), and CD86 median fluorescence intensity (MFI) as a measure of activation (figure 5.2B).

Previously, Ochiai et al. ${ }^{143}$ showed that after one treatment of DBP-FITC on the ear skin tissue of mice, CDllb ${ }^{+}$DCs and TSLP-dependent TN DCs were essential for induction of Th2 inflammation in this model. These subsets had expanded most, had the highest frequency of FITC $^{+}$DCs and had the highest CD86 expression. ${ }^{143}$ Coinciding with this study, one application of DBP-FITC caused inflammation (increased total cell number) in the auricular LNs (figure 5.3A). Furthermore, there was a significant reduction of total live immune cells present in the auricular LNs of infected mice in comparison to uninfected mice after DBP-FITC treatment. In addition, $\mathrm{CD} \mathrm{bb}^{+}$and TN DC subsets showed the highest numbers of $\mathrm{FITC}^{+}$cells (figure 5.3B). However, infected mice only showed a slight reduction in ITC $^{+}$DCs in each subset, which did not reach significance.

Finally, while the TN DC subset had the highest fold increase of CD86 expression, coinciding with the results from Ochiai et $a l,{ }^{143}$ there was no difference between uninfected mice and infected mice (figure 5.3C). 


\subsubsection{H. polygyrus suppresses inflammation and induces IL-4-producing Th2 cells and Tregs in the auricular LNs}

Tregs have been shown to suppress Th2 inflammation in tissues such as the lung, and to be important for the regulation of inflammation in AD. ${ }^{13}{ }^{160}$ As Tregs were induced after HDM-induced skin inflammation in the auricular LN and ear skin tissue, it is likely that a similar phenotype would be seen during a different AD model.

To determine if $H$. polygyrus is able to suppress lymphocytes in the auricular LNs after DBP-FITC treatment, C57BL/6 mice were infected with 200 L3 larvae via oral gavage at day -14 , treated with DBP-FITC on day 0 and day 2 , and analysed on day 7 (figure 5.4A). Immune cells isolated from both auricular LNs (supplementary figure 1) were taken for analysis by flow cytometry and one ear was taken for RNA extraction for analysis of gene expression by qPCR. Immune cells isolated from the LNs were analysed for the presence of $\mathrm{B}$ cells $\left(\mathrm{CD19} 9^{+}\right), \mathrm{CD}^{+} \mathrm{T}$ cells $\left(\mathrm{CD} 3^{+} \mathrm{CD} 8^{+}\right)$, $\mathrm{CD}^{+} \mathrm{T}$ cells $\left(\mathrm{CD}^{+} \mathrm{CD}^{+}\right)$, Tregs $\left(\mathrm{FoxP}^{+} \mathrm{CD}^{+} \mathrm{T}\right.$ cells), and Th2 cells (GATA3 ${ }^{+} \mathrm{CD} 4^{+}$ T cells).

Coinciding with previous results, DBP-FITC induces expansion of immune cells in the auricular LNs, and there is a significant reduction of immune cells present in $H$. polygyrus-infected mice (figure $5.4 \mathrm{~B}$ ). This significant reduction of immune cells also extends to numbers of $\mathrm{B}$ cells (figure 5.4C), $\mathrm{CD}^{+} \mathrm{T}$ cells (figure 5.4D), and $\mathrm{CD}^{+} \mathrm{T}$ cells (figure $5.4 \mathrm{D}$ ), although these cell proportions are unchanged (data not shown).

While the proportion of $\mathrm{CD}^{+} \mathrm{T}$ cells in the $\mathrm{LN}$ are unchanged by infection after DBP-FITC treatment, proportions of $\mathrm{T}$ cell subsets have changed. FoxP3 ${ }^{+}$Tregs (figure 5.5A), and GATA3 $^{+}$Th2 cells (figure 5.5B) are significantly increased in proportion in infected mice, although this increase in Tregs does not translate to absolute numbers and is still significantly lower in $H$. polygyrus-infected mice, reflecting the size of the LNs. These results indicate that $\mathrm{CD} 4^{+} \mathrm{T}$ cells have various subsets that change in proportion in $H$. polygyrus-infected mice after DBP-FITC 
treatment and there might be other subsets of T cells that are influenced by $H$. polygyrus.

Previous results have shown that $H$. polygyrus induces $\mathrm{IL}_{-} 4^{+} \mathrm{T}$ cells in various tissues, including in the ear skin tissue (figure 3.1), while $H$. polygyrus suppresses IL$4^{+} \mathrm{T}$ cells (likely Tfh cells) in the auricular LNs after ID HDM injection (figure 4.4D).

For this section, immune cells isolated from the auricular LNs were cultured and stimulated for 4 hours in the presence of Brefeldin A, PMA, and Ionomycin. B cells and $\mathrm{CD} 4^{+} \mathrm{T}$ cells were analysed for their expression of Th2 cytokines IL-4, IL-10, and IL-13.

IL-4 was produced by both B cells and $\mathrm{CD}^{+} \mathrm{T}$ cells in the $\mathrm{LN}$, and in contrast to the HDM model, cells producing IL-4 were significantly increased in proportions in $\mathrm{H}$. polygyrus-infected mice (figure $5.5 \mathrm{C}, \mathrm{E}$ ). IL-10 was also produced by both B cells and $\mathrm{T}$ cells (figure 5.5D, F), and while proportions were no different, absolute numbers were significantly decreased in infected mice. This suggests suppression of inflammation by $H$. polygyrus is not dependent on IL-10, which corresponds with previous data from Wilson et al. ${ }^{13,14}$ IL-13-producing lymphocytes were not present in the auricular LNs (data not shown), and more likely were present in the ear skin tissue during the peak of inflammation.

\subsubsection{H. polygyrus suppresses $/ / 13$ gene expression during peak of inflammation}

Previous studies have shown that $H$. polygyrus suppressed HDM-induced lung inflammation and pro-inflammatory cytokine expression in the lungs. ${ }^{13}$ Furthermore H. polygyrus was able to block IL-33 expression in a model of OVA-Alternariainduced lung inflammation, suppressing expansion of and cytokine production by ILC2s. ${ }^{123}$ While $H$. polygyrus suppresses inflammation and expansion of lymphocytes in the auricular LNs, cytokine profiles in the ear skin tissue might be altered, as illustrated by intracellular cytokine staining results above. Therefore, from the same experiment RNA was extracted from the ear skin tissue of mice at 
day 7 post-DBP-FITC treatment, according to protocol for gene expression analysis by qPCR.

Tslp expression, which had been demonstrated to be crucial for the initiation of the Th2 response, ${ }^{214}$ was increased after DBP-FITC treatment as expected, although was only slightly increased during the peak of inflammation (figure 5.6A), suggesting an earlier time point would be needed to determine Tslp levels directly after DBP-FITC treatment. Although Tslp levels are decreased in $H$. polygyrus-infected mice, this difference did not reach significance (figure 5.6A). Previous results have demonstrated $H$. polygyrus increases $\mathrm{IL}-4^{+} \mathrm{T}$ cells in the ear skin tissue. However, after DBP-FITC treatment, Il4 expression increased profoundly and $H$. polygyrus did not alter this high level (figure 5.6B). Conversely, while Ill3 expression increased after DBP-FITC treatment, it was significantly decreased in $H$. polygyrus-infected mice (figure 5.6C), suggesting Ill3 gene expression is more susceptible to the suppressive effects of $H$. polygyrus than Il4.

Intriguingly, expression of the Treg marker Foxp3 was decreased in H. polygyrusinfected mice compared to uninfected mice after DBP-FITC treatment (data not shown), and no difference was found in expression of anti-inflammatory cytokines IllO and Tgf (data not shown). These results suggest during the peak of the inflammation there is no increase in Tregs or anti-inflammatory cytokines in $H$. polygyrus-infected mice, and an alternative mechanism of suppression may be induced by the parasite, at least in this setting. 
A

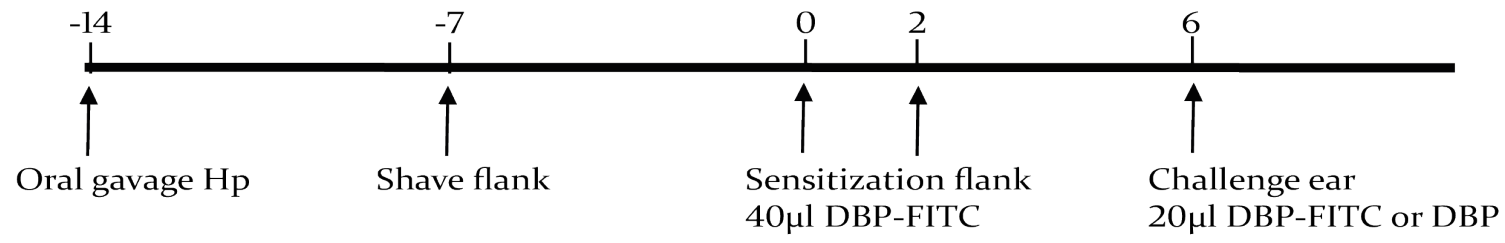

B

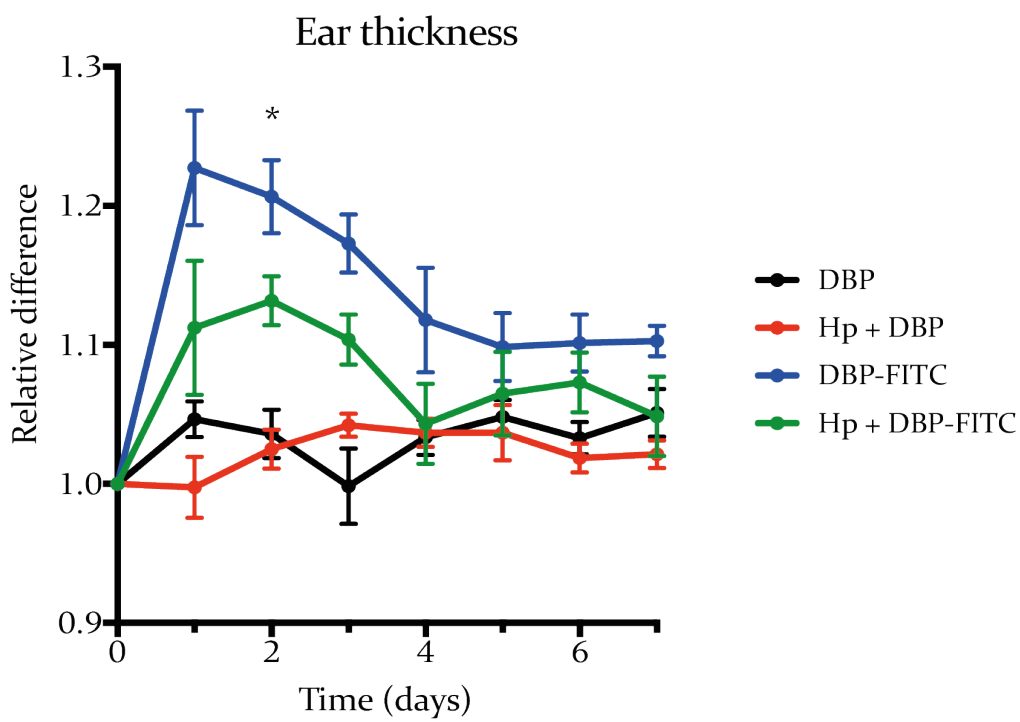

C

Transepidermal Water Loss

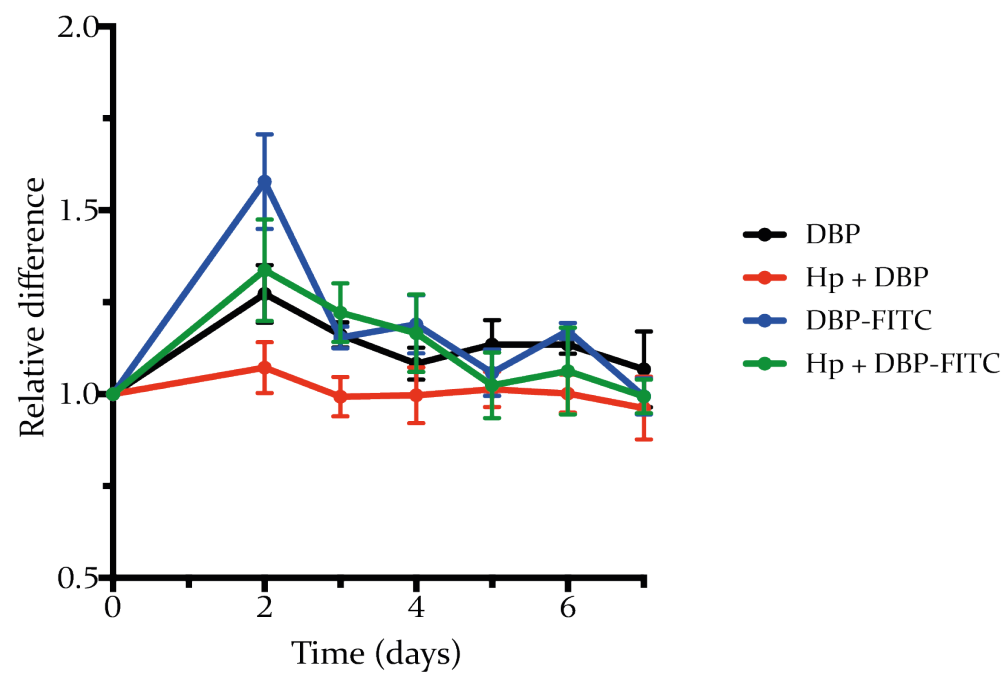

Figure 5.1: H. polygyrus suppresses inflammation in a model of DBP-FITC-induced CHS.

A) Model for $H$. polygyrus infection, sensitization with DBP-FITC and challenge with DBP-FITC or DBP (numbers depict the day). Relative increase of B) ear thickness and C) TEWL in comparison to baseline (before challenge on ear skin tissue) Graphs show mean \pm SEM, statistical significance was determined with a Student's unpaired T-test. * $\mathrm{p}<0.05$. 
A

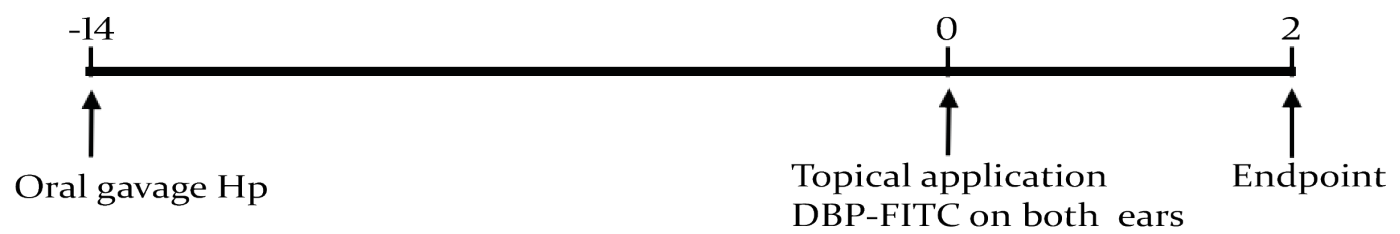

B

CD45+ cells B220-
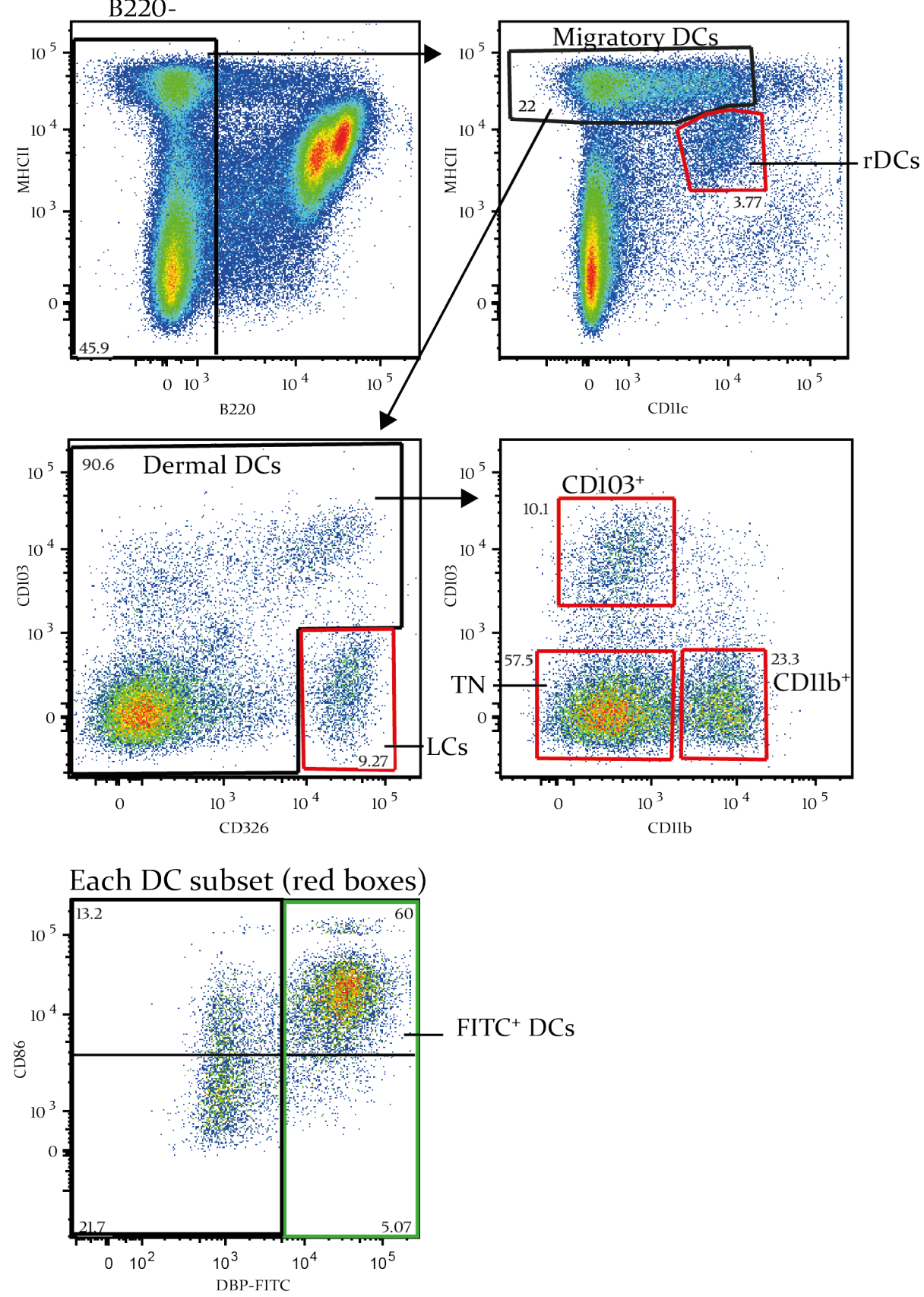

Figure 5.2: Model and gating strategy for analysing DC subsets after treatment with DBPFITC.

A) Model for $H$. polygyrus infection and treatment with DBP-FITC (numbers depict the day). B) Gating strategy for 5 specific DC subsets in the auricular LNs: resident DCs (rDCs), Langerhans cells (LCs), CD1lb ${ }^{+}$DCs, CD103 ${ }^{+}$DCs and TN DCs. From each DC subset frequency of ITC $^{+}$cells and median fluorescence intensity (MFI) of CD86 was determined. 
A

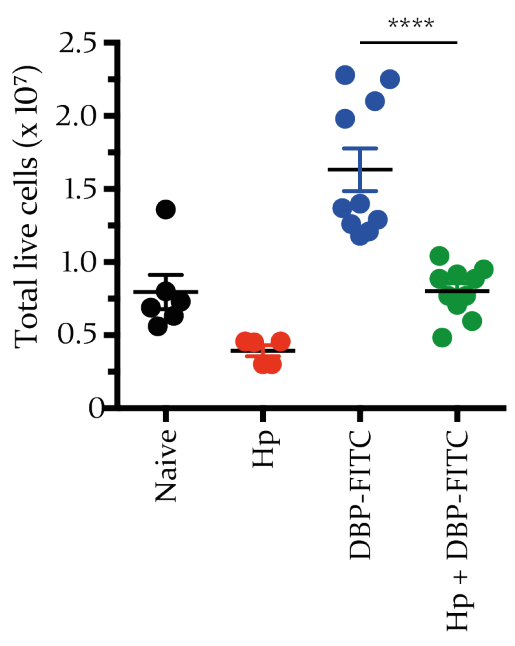

C

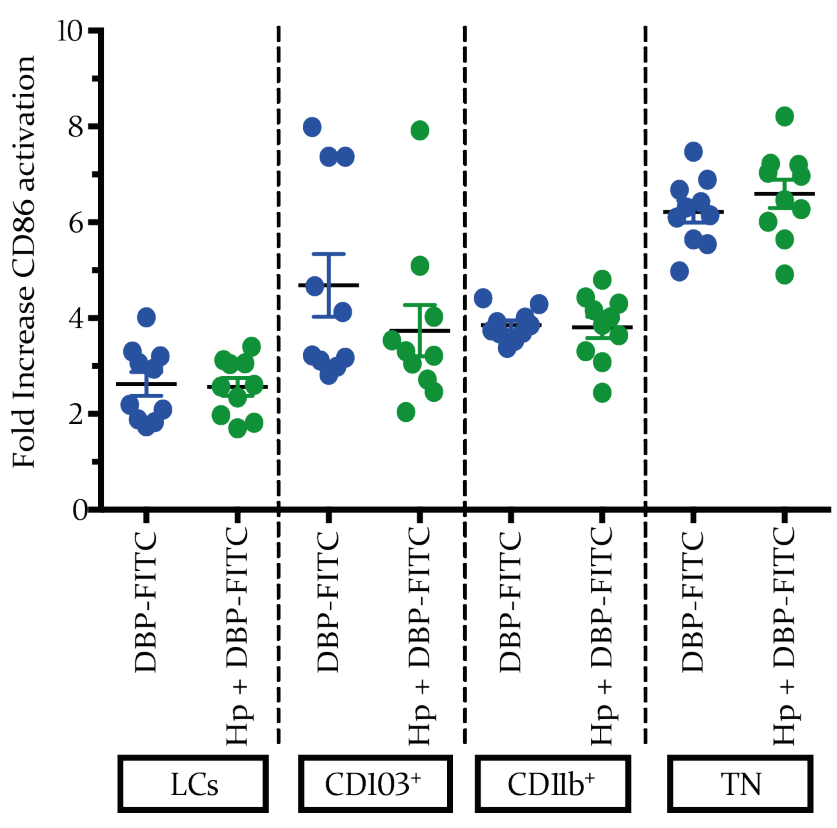

Figure 5.3: H. polygyrus suppresses early inflammation after DBP-FITC treatment.

A) Total immune cells and B) FITC ${ }^{+}$DCs from each DC subset present in the auricular LN. C) Fold increase of CD86 MFI in $\mathrm{FITC}^{+}$DCs in relation to CD86 MFI in FITC ${ }^{-}$DCs for LCs, $\mathrm{CD}^{-103^{+}}$DCs, $\mathrm{CD} \mathrm{Cb}^{+} \mathrm{DCs}$ and TN DCs. Data was collected from two separate experiments. Graphs show mean ${ }_{ \pm}$SEM, statistical significance was determined with a Student's unpaired T-test. ${ }^{*} \mathrm{p}<0.05,{ }^{* *} \mathrm{p}<0.01$, ${ }^{* * *} \mathrm{p}<0.001,{ }^{* * * *} \mathrm{p}<0.0001$. 
A

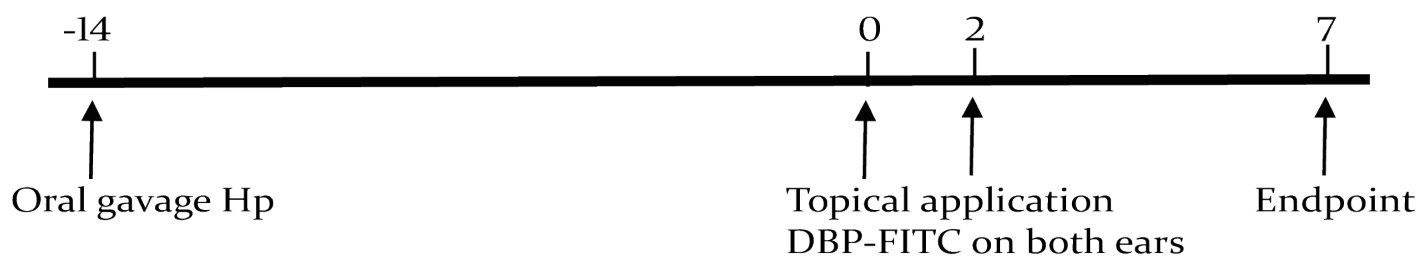

B

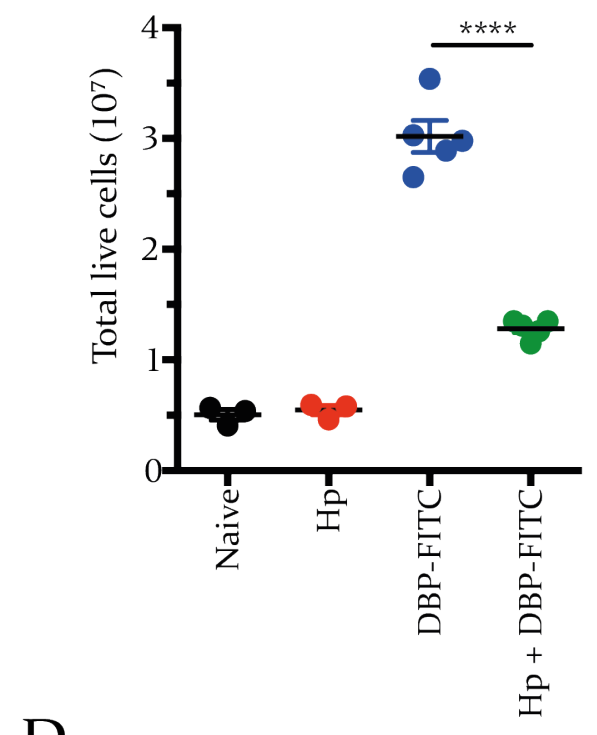

$\mathrm{D}$

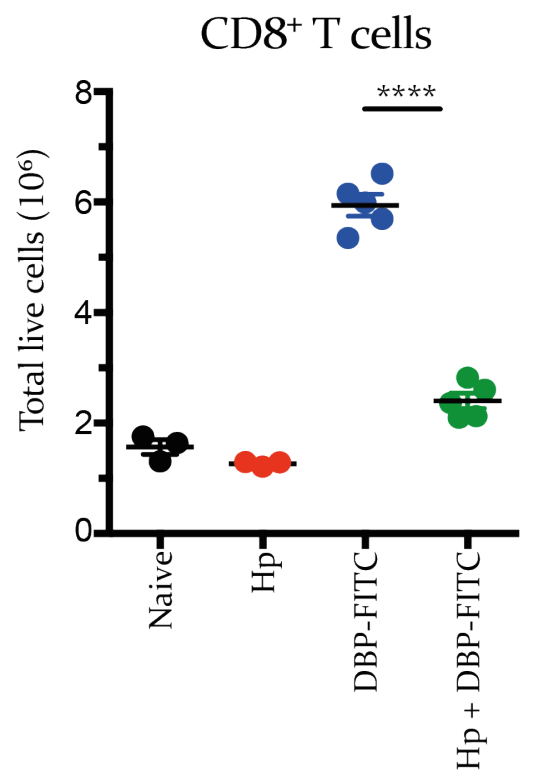

C

B cells

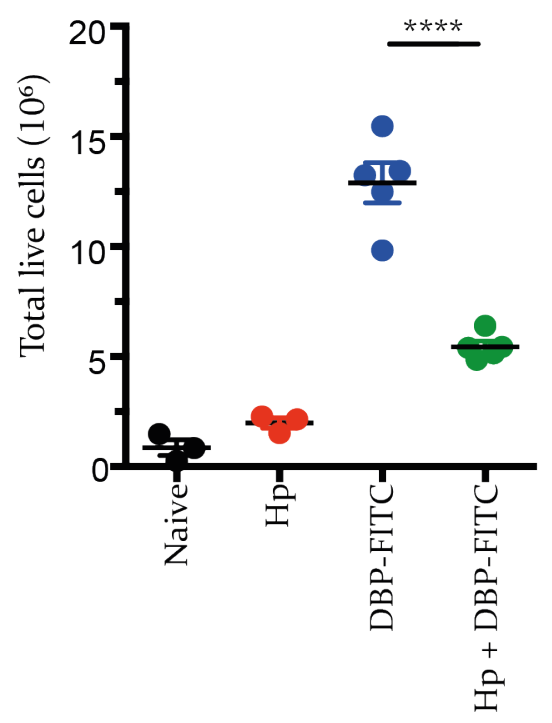

$\mathrm{E}$
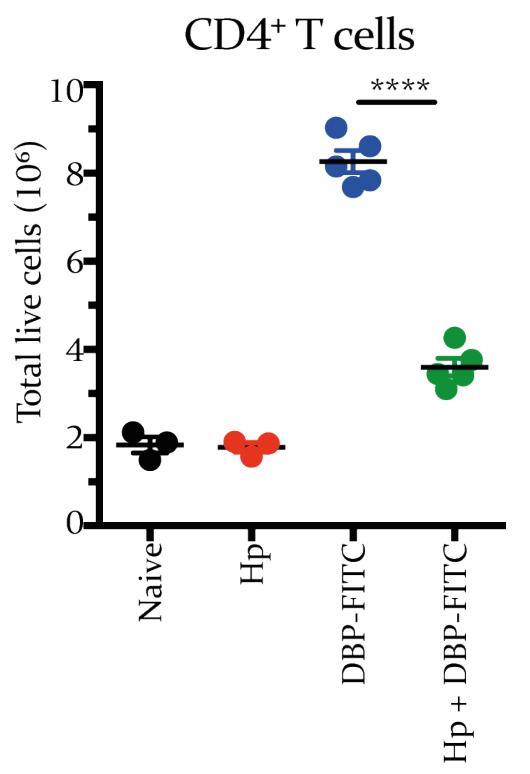

Figure 5.4: H. polygyrus suppresses inflammation in the auricular LNs during the peak of inflammation after DBP-FITC treatment.

A) Model for H. polygyrus infection and treatment with DBP-FITC (numbers depict the day). B) Total live immune cells, C) total live B cells, D) $\mathrm{CD}^{+} \mathrm{T}$ cells, and E) CD4 ${ }^{+} \mathrm{T}$ cells present in the auricular LNs. Graphs show mean \pm SEM, statistical significance was determined with a Student's unpaired Ttest. ${ }^{*} \mathrm{p}<0.05,{ }^{* *} \mathrm{p}<0.01,{ }^{* * *} \mathrm{p}<0.001,{ }^{* * * *} \mathrm{p}<0.0001$. 
A

FoxP3 ${ }^{+}$Tregs

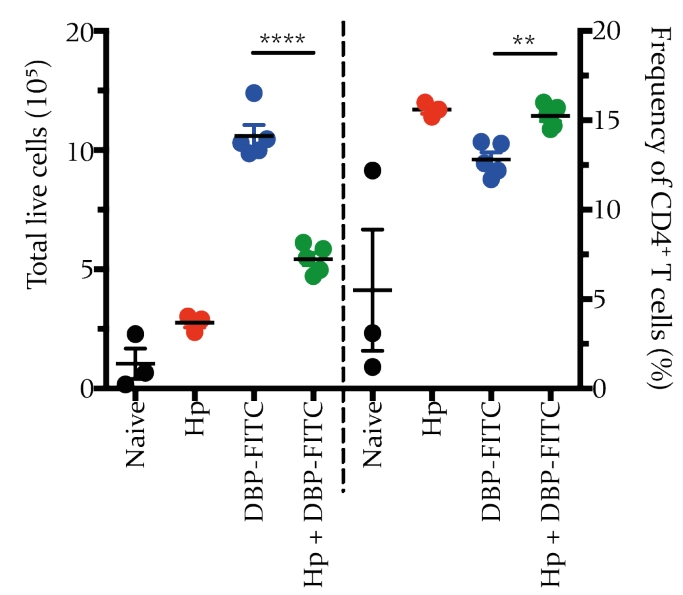

C

IL-4+ B cells

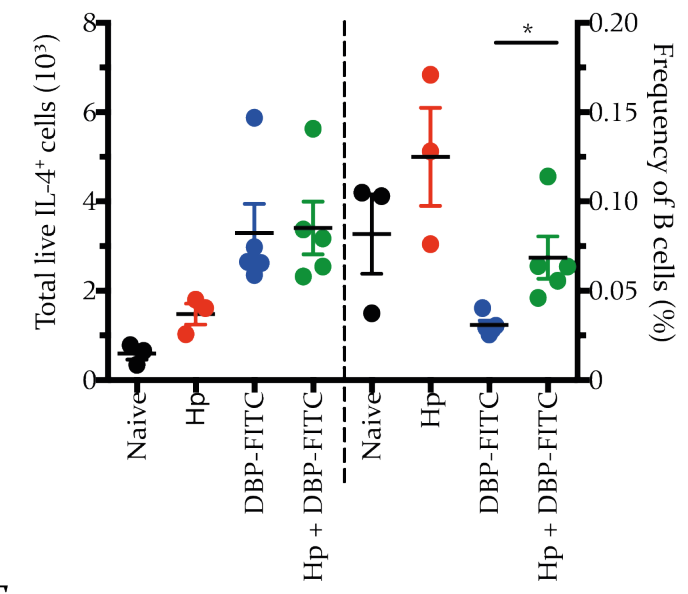

E IL- $4^{+} \mathrm{CD} 4^{+} \mathrm{T}$ cells

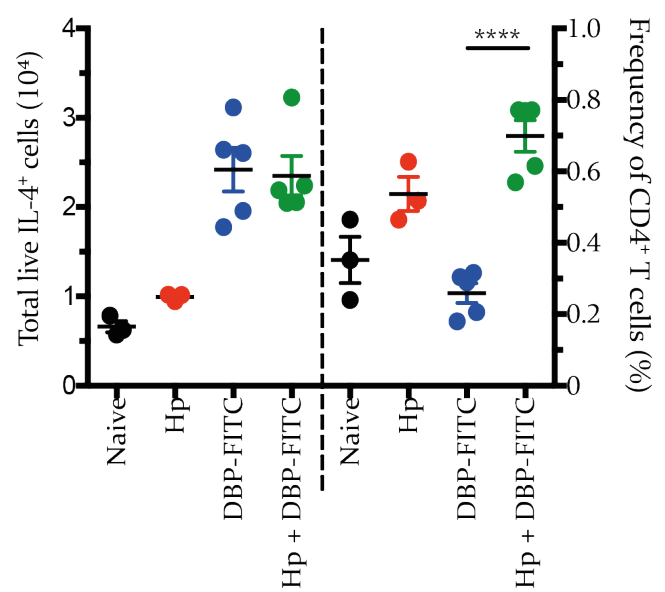

B

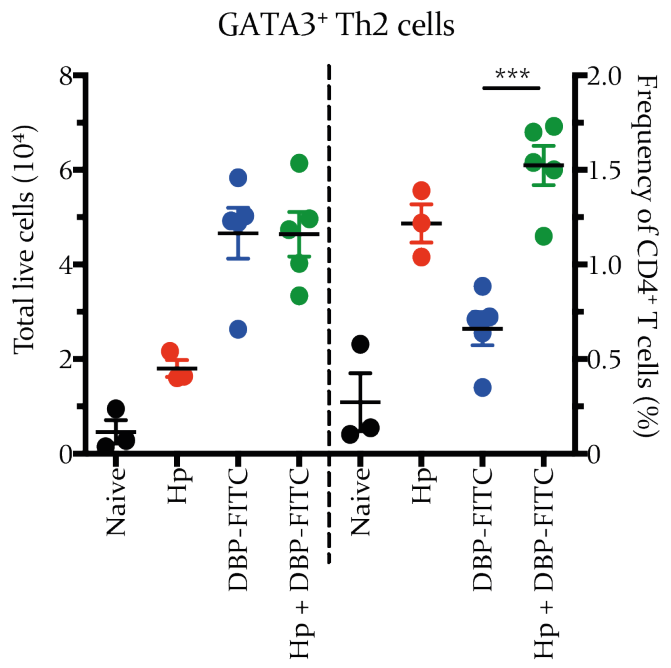

$\mathrm{D}$

IL- $10^{+} \mathrm{B}$ cells

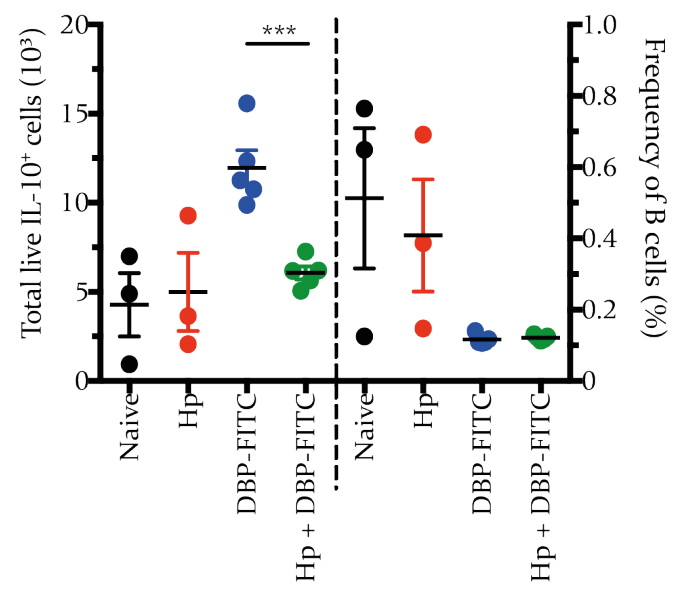

IL- $10^{+} \mathrm{CD} 4^{+} \mathrm{T}$ cells

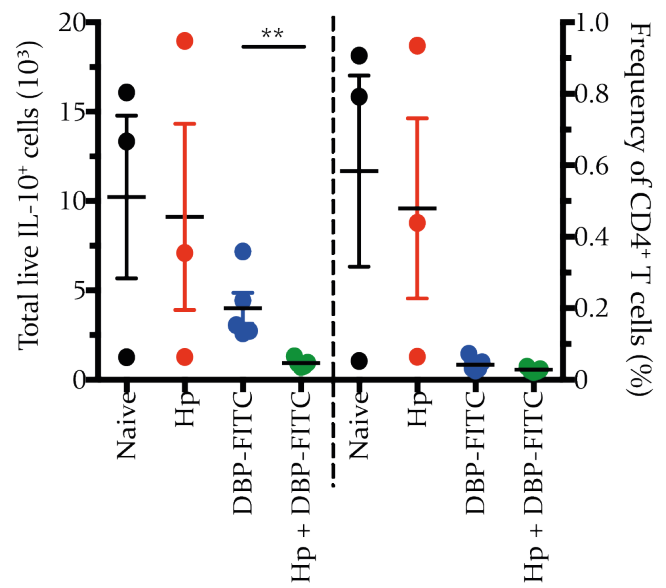

Figure 5.5: $H$. polygyrus induces Tregs and $\mathrm{IL}_{-} \mathrm{4}^{+} \mathrm{T}$ cells in the auricular $\mathrm{LN}$ after DBP-FITC treatment.

A) Total live FoxP3 ${ }^{+}$Tregs and B) total live GATA3 ${ }^{+}$Th2 cells in the auricular LNs. C) IL- $4^{+}$and D) IL$10^{+} \mathrm{B}$ cells in the auricular LNs. E) $\mathrm{IL}_{-} 4^{+}$and F) $\mathrm{IL}^{-10^{+}} \mathrm{CD} 4^{+} \mathrm{T}$ cells in the auricular LNs. Graphs show mean \pm SEM, statistical significance was determined with a Student's unpaired T-test. ${ }^{*} \mathrm{p}<0.05$, ** $\mathrm{p}<0.01,{ }^{* * *} \mathrm{p}<0.001,{ }^{* * * *} \mathrm{p}<0.0001$. 
A

Tslp

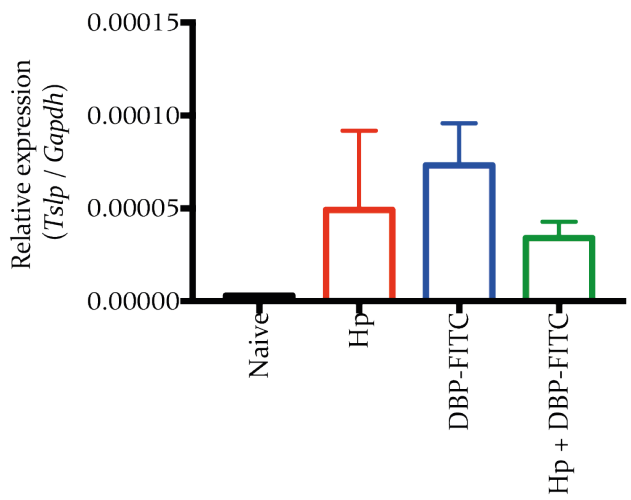

C

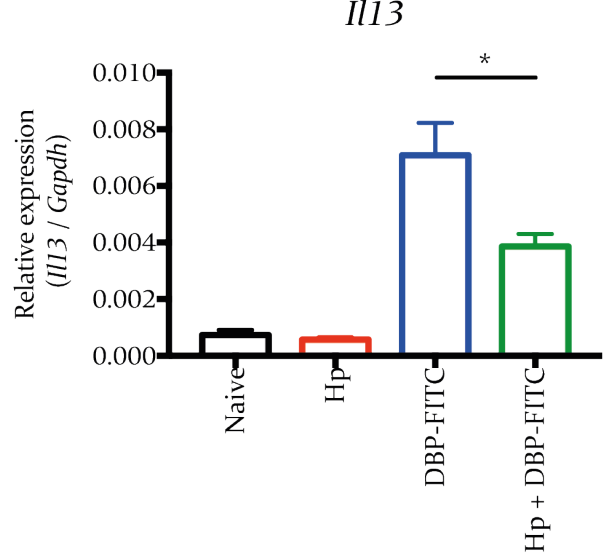

B

Il4

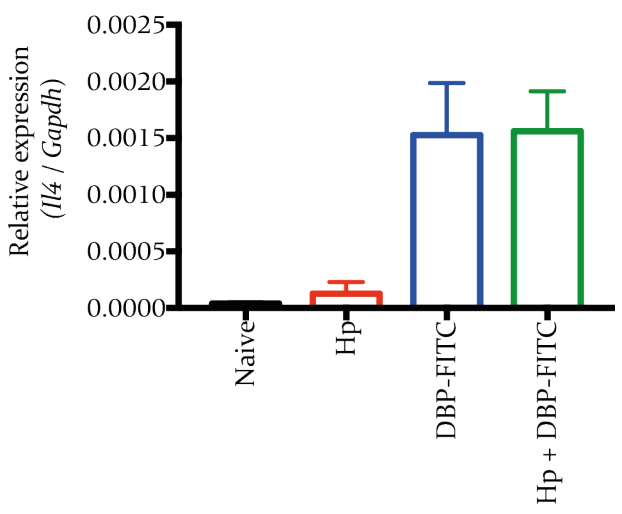

Figure 5.6: H. polygyrus suppresses Ill3 during the peak of inflammation.

Gene expression of A) Tslp, B) Il4, and C) Ill3 relative to that of Gapdh. Graphs show mean \pm SEM, statistical significance was determined with a Student's unpaired T-test. ${ }^{*} \mathrm{p}<0.05$. 


\subsection{Discussion}

Previous studies have shown DBP-FITC is a suitable model for AD, and is characterized by keratinocyte secretion of TSLP, which activates CDIlb $^{+}$and TN subsets of dermal DCs, which migrate to the skin-draining LNs and activate Th2 cells. ${ }^{143,162,214}$ The adaptive arm of the immune system has been shown to be crucial for this model and innate cells in the skin were shown to ameliorate inflammation. ${ }^{215}$ To date, there is no data on the effects of helminth infection on DBP-FITC-induced AD.

The results above have demonstrated $H$. polygyrus is able to suppress skin inflammation in the ear tissue using a model of CHS, as ear thickness was significantly reduced during the peak of inflammation. There was no difference in TEWL, suggesting $H$. polygyrus is able to suppress inflammation in this model of $\mathrm{AD}$, while it has no influence on skin barrier function. Histological staining of sections of ear tissue could be analysed to indicate specific immune cell subsets that are affected by infection. Also, analysis of specific molecules known to be involved in $\mathrm{AD}$, such as filaggrin and tight junction proteins, could be undertaken to assess if helminth infection affects expression over the time course of inflammation. ${ }^{156,208,216}$

Next, as $H$. polygyrus is known to induce tolerogenic DCs in the gut and block migration of pro-inflammatory DCs to the MLNs, it was hypothesized that $H$. polygyrus might be able to influence DCs in the ear skin tissue as well. ${ }^{128}$ Therefore, a model was used consisting of one treatment of DBP-FITC on the ear skin tissue to study migration of different DC subsets. While total live immune cells were significantly suppressed in the auricular LNs by $H$. polygyrus infection, there was no significant reduction in absolute FITC $^{+}$DC numbers in the auricular LNs, and no change in CD86 expression. These results suggest $H$. polygyrus suppresses expansion of immune cells in the auricular LNs at an early time point, but does not alter DC phenotype according to the limited panel of markers used. Culturing these DCs in vitro with T cells would demonstrate if DC function is altered by $H$. polygyrus. Furthermore, $H$. polygyrus might block migration of DC into the 
auricular LNs, and studying migratory markers could provide evidence for this hypothesis.

Previous results demonstrated Tregs were increased in proportion after HDMinduced Th2 inflammation during the peak of inflammation. A simplified model of DBP-FITC was used to induce Th2 inflammation in the ear skin tissue, and the auricular LNs were analysed during the peak of inflammation (day 7). In accordance with previous results, total live immune cells were significantly reduced in the auricular LNs during the peak of inflammation. While absolute $\mathrm{CD} 4^{+} \mathrm{T}$ cell numbers were significantly reduced, there was no change in $\mathrm{CD}^{+} \mathrm{T}$ cell proportion. However, within the $\mathrm{CD} 4^{+} \mathrm{T}$ cell population, there were some significant changes in cell subsets in $H$. polygyrus-infected mice. Both FoxP3 ${ }^{+}$Treg and GATA3 $^{+} \mathrm{T}$ cell proportions were significantly increased, suggesting other $\mathrm{T}$ cell subsets might be suppressed to compensate. Further studies including staining for other transcription factors such as Tbet for Thl cells, and markers to look at activated or naive T cells such as CD44 and CD62L, might provide more information on $\mathrm{CD}^{+} \mathrm{T}$ cell proportions in the LNs in $H$. polygyrus-infected mice.

Isolated immune cells from this experiment were also cultured and stimulated in the presence of Brefeldin A, PMA, and Ionomycin and stained intracellularly for the cytokines IL-4, IL-10, and IL-13. While IL-4 $4^{+} \mathrm{B}$ cell and $\mathrm{T}$ cell proportions (but not absolute numbers) were significantly increased, $\mathrm{IL}_{-}-10^{+} \mathrm{B}$ cell and $\mathrm{T}$ cell numbers (but not proportions) were significantly decreased in $H$. polygyrus infected mice. However, as proportions of $\mathrm{IL}_{-} 4^{+}$and $\mathrm{IL}-10^{+}$cells in both $\mathrm{B}$ cells and $\mathrm{T}$ cells were very low (below $1 \%$ of $\mathrm{B}$ and $\mathrm{CD}^{+} \mathrm{T}$ cells), this difference might not be biologically significant.

Finally, using the ear skin tissue, RNA was extracted for gene expression analysis by qPCR. During the peak of inflammation there was low expression of Tslp, and expression was slightly decreased in $H$. polygyrus-infected mice. An earlier time point may be more appropriate to assess Tslp gene expression in this model. As Tslp is important for the induction of Th2 inflammation in this model, this suggests a decrease of this cytokine could inhibit downstream activation of Th2 cells. The canonical Th2 cytokines Il4 and Ill3 were both profoundly increased after DBP-FITC 
treatment on the ear skin tissue. Earlier results showed IL- $4^{+}$Th2 cells were significantly increased in ear skin tissue in $H$. polygyrus-infected mice, qPCR results here demonstrate $I l 4$ was slightly increased in $H$. polygyrus-infected mice, but does not reach the level of expression DBP-FITC treatment induces. While Il4 was unchanged in $H$. polygyrus-infected mice, Ill3 was significantly decreased, which suggests $I l 13$ might be more susceptible to the suppressive effects of $H$. polygyrus. Surprisingly, Foxp3 expression was significantly reduced in $H$. polygyrus-infected mice, and expression of IllO and Tgf $\beta$ was not significantly altered. These results suggest that genes of regulatory markers might be expressed before the peak of inflammation, and would require further experiments for validation. Also, other mechanisms of suppression by $H$. polygyrus cannot be ruled out.

The results above have demonstrated $H$. polygyrus is able to suppress Th2 inflammation in the ear skin tissue induced by DBP-FITC treatment (i.e. illustrated by reduced ear thickness and a decrease in total live cells in the auricular LNs). While Tregs are increased in the auricular LNs at the peak time, further study is required to validate the importance of Tregs for the suppressive effects of $H$. polygyrus. Depletion of Tregs in $H$. polygyrus-infected mice and adoptive transfer of these Tregs into uninfected mice would further demonstrate the importance of Tregs in suppression of Th2 inflammation in the ear skin tissue. 


\subsection{Conclusions}

Keypoints:

1) H. polygyrus suppresses inflammation in the ear skin tissue, but does not seem to alter skin barrier function.

2) DC phenotype is not altered by $H$. polygyrus infection.

3) H. polygyrus suppresses inflammation and expansion of lymphocytes in the auricular LNs, coinciding with increased proportions of Tregs and Th2 cells.

4) H. polygyrus suppresses Ill3 expression in the ear skin tissue, and might suppress Tslp expression during the peak of inflammation.

5) Further research is required to determine the importance of Tregs for suppression of Th2 inflammation in the ear skin tissue by H. polygyrus. 
Chapter 6. Final discussion 


\subsection{Discussion}

Helminths infect over 1 billion people in the world. ${ }^{22}$ They cause a variety of symptoms, most importantly for quality of life being anaemia and growth deficiencies, and there are complications that arise when people are co-infected with other microbes such as HIV and Mycobacterium. ${ }^{31-33}$ Failure to control coinfections is often due to the skewing from a Thl/Thl7-mediated immune response needed for protection against many microbes, to a Th2-mediated immune response elicited by helminths, leading to impaired anti-viral and anti-bacterial immunity. ${ }^{38}$ For these reasons, the eradication of helminths would be desirable in developing countries. However, an inverse correlation is very often found between immune disorders and helminth infections; in Western countries where people are not chronically infected with helminths autoimmune and allergic disorders are an increasing problem, while people in developing countries, where helminth infection is endemic, have a reduced risk of developing autoimmune or allergic diseases. ${ }^{2,4,9}$, 12 This inverse correlation has led to the proposal of the hygiene hypothesis, which further states that early exposure to environmental allergens could also protect against the development of atopy in humans. ${ }^{2,4}$

Helminths induce a strong immune response in the host mediated by Th2 cells, innate cells such as eosinophils, ILC2s, AAMs and mast cells, and Th2 cytokines IL4, IL-5, IL-9 and IL-13. Due to many millennia of parasite:host co-evolution, it is believed the Th2 response has evolved to expel or contain helminths, while also repairing and limiting any parasite- and immune-mediated tissue damage. ${ }^{24,51}$ However, helminths are highly adapted to the host immune system and have been shown to induce regulatory mechanisms that suppress inflammation and limit tissue damage, ensuring prolonged survival and reproduction of the worm, but also survival of the host. ${ }^{15,24}$ This induction of regulatory mechanisms by helminths was also found to be protective against allergic and autoimmune diseases and currently clinical trials have found the pig whipworm T. suis and human hookworm $N$. americanus to be safe and effective in suppressing symptoms in IBD such as Crohn's disease, and MS. ${ }^{18-21}$ 
$H$. polygyrus is a gut-dwelling natural mouse parasite, and is highly adapted to the mouse immune system, making it the preferred model to study chronic helminth infection in mice. $H$. polygyrus secretes several hundred different molecules into its host, collectively termed HES. ${ }^{94,}$ 95, 217 These molecules are believed to be the underlying factors responsible for the induction of regulatory immune mechanisms, with HES alone being enough to suppress Th2 allergic inflammation, without live worm infection. ${ }^{13,15,123}$ Several studies have focused on the content and function of HES, to isolate the components responsible for the worm's suppressive effects. ${ }^{15,94,}$ 95

Regulatory mechanisms induced by $H$. polygyrus include cells such as Tregs, Bregs and AAMs, and the secretion of anti-inflammatory cytokines, e.g. IL-10 and TGF $\beta$ (figure 1.3). ${ }^{13,14,113,218}$ Bregs are increased in the gut after infection with $H$. polygyrus and, Tregs are also induced in the lung suppressing HDM-induced lung inflammation. ${ }^{13,14}$ Adoptive transfer of these cells to uninfected mice transfers protection against Th2-mediated lung inflammation, while depletion of Tregs abolishes protection, suggesting Tregs are central to this helminth-induced immune suppression. ${ }^{13,14}$

While $H$. polygyrus was found to suppress the adaptive arm of the immune system through inducing a tolerogenic phenotype of CDllc ${ }^{\text {lo }}$ DCs, which were capable of inducing Tregs and IL-10 production, while blocking migration of pro-inflammatory CD1lc $^{\text {hi }}$ DCs to the MLN. ${ }^{127,128}$ Furthermore, H. polygyrus was also able to inhibit the innate arm of the immune system in the lungs through suppression of IL-33 release from epithelial cells, which blocked expansion of ILC2s, and as ILC2s are the main source of IL-5 and IL-13 in several models of lung inflammation, recruitment and activation of downstream effector cells such as eosinophils were also suppressed. ${ }^{123}$

While several helminths have been shown to be protective against Th2-mediated inflammation in the lungs and guts, there is limited knowledge of the effect of helminth infections during skin allergy, and this area is in need of supplementation. $\mathrm{AD}$ is a chronic/chronically relapsing allergic disorder, characterized by increased TSLP production, ${ }^{152,213}$ and impaired skin barrier function, ${ }^{173}$ leading to Th2mediated inflammation and possible IgE sensitization to other allergens, ${ }^{170}$ leading 
to the allergic march. ${ }^{175}$ The skin has been shown to be essential for the sensitization of allergens and initiation of the allergic march, ${ }^{174}$ and the prevention of the onset of AD would also be able to prevent the onset of other allergies later in life. With this in mind, the overall aim of this project was to study if the gut dwelling mouse parasite $H$. polygyrus was able to suppress Th2-mediated inflammation in the skin tissue.

H. polygyrus infection induces $\mathrm{IL}^{-} 4^{+} \mathrm{Th} 2$ cells in the blood and various organs including the spleen and lungs. ${ }^{179}$ However, there was no data included in previous studies on whether this also occurred in the skin tissue. Over-expression of IL-4 changes steady-state conditions in the skin, resulting in delayed wound healing, mast cell accumulation and keratinocyte hyperproliferaton. ${ }^{166,180}$ However, IL-4 by itself cannot induce AD. ${ }^{180}$ Furthermore, IL-4 production in the skin tissue is sufficient for the induction of AAMs, which can secrete anti-inflammatory cytokines IL-10 and TGF $\beta$. Another canonical Th2 cytokine IL-13 is constitutively expressed in the skin by ILC2s, and is important for the maintenance of the skin barrier and for wound repair. ${ }^{149,181}$ Therefore, I used 4Cl3R mice to study the effect of $H$. polygyrus infection on expression of canonical Th2 cytokines IL-4 and IL-13, in several tissues including the skin tissue.

Confirming research by Mohrs et al. ${ }^{179}$, I found there was an induction of IL- $4^{+} \mathrm{T}$ cells in tissues distal to the gut, including in the ear skin tissue (chapter 3.3). IL-4 was also produced by innate cells such as eosinophils and macrophages, although to a lesser degree. Previous studies showed that rapid production of IL-4 after tissue injury by innate cells is sufficient for the induction of AAMs, although IL-4 from Th2 cells is necessary for the maintenance and full activation of AAMs. ${ }^{183}$ Therefore, importantly my results also suggest that the stimulus for induction and maintenance of AAMs is present in the skin of infected mice, which could lead to suppression of skin inflammation. Further studies are needed to determine if AAMs are induced in the skin after infection with $H$. polygyrus and if they are producing anti-inflammatory cytokines IL-10 and TGF $\beta$, which could lead to induction of Tregs and further suppression of inflammation. 
The first model I used to induce Th2 inflammation in the ear skin tissue utilised ID injection of whole mashed-up HDM. ${ }^{200} \mathrm{HDM}$ induces strong Th2-mediated inflammation through activation of TLRs, CLRs and NLRs, but in this model HDM does not induce TSLP expression by keratinocytes. ${ }^{143,190-193}$ I found immune cells including (IL-4 producing) T cells, B cells, eosinophils, and neutrophils were present after HDM administration, with the peak of cellular inflammation at day 7 after injection (chapter 4.3.1).

I observed $H$. polygyrus suppressed HDM-induced Th2 inflammation in the ear draining LNs and ear skin tissue, coinciding with an increased proportion of Tregs in both sites (chapter 4.3.2 and 4.3.3). Furthermore, H. polygyrus induced AAMs in the ear skin tissue above naive levels, although no difference was seen between uninfected and $H$. polygyrus-infected mice after HDM injection. My results suggest suppression is likely to be mediated by Tregs, which coincides with previous studies showing $H$. polygyrus suppressed HDM-induced lung inflammation. ${ }^{13}$ Future experiments could utilise depletion of Tregs in $H$. polygyrus-infected mice or adoptively transferring them to uninfected mice to investigate if protection against HDM-induced Th2 inflammation in the ear skin tissue is indeed dependent on Tregs. Furthermore, a larger sample size would be necessary to prove if AAMs are induced and play a role in this model.

The second model I used to induce Th2 inflammation in the ear skin tissue was the topical application of DBP-FITC. DBP-FITC-induced CHS is initiated through the production of TSLP by keratinocytes. ${ }^{162,212,214}$ This model has been shown to be dependent on Th2 cells activated by DCs, with innate cells such as mast cells and eosinophils in the ear skin tissue enhance inflammation. ${ }^{215}$ My results have demonstrated that $H$. polygyrus was suppressive in this model of DBP-FITC-induced CHS with infected mice having reduced ear thickness after challenge with DBPFITC on the ear skin tissue (chapter 5.3.1). I found no significant difference in TEWL however, indicating $H$. polygyrus suppresses ear inflammation, but does not affect skin barrier function in this model. Future experiments should include the further study of inflammation in the ear skin tissue by analysing the infiltration of different 
immune cells in to the skin, and skin barrier integrity by histology and real-time PCR.

H. polygyrus induces a phenotype of tolerogenic CDllc ${ }^{\text {lo }}$ DCs in the gut, which are able to induce Tregs, and block migration of pro-inflammatory CD1lc ${ }^{\text {hi }}$ DCs to the LNs. ${ }^{128}$ As DCs are important for the initiation of Th2 inflammation in this model, I hypothesized $H$. polygyrus might be able to induce suppression of the adaptive arm through blocking DC migration to the LNs, or inducing a tolerogenic phenotype. My results indicated that $\mathrm{CDIlb}^{+}$DCs and TN DCs expanded most after DBP-FITC treatment and had the highest proportion of $\mathrm{FITC}^{+}$DCs, i.e. DCs activated by DBPFITC, coinciding with results found by Ochiai et al. ${ }^{143}$ At this early time point, I observed $H$. polygyrus suppressed expansion of immune cells in the ear draining LNs, although the absolute DC numbers were only slightly decreased and this difference did not reach significance (chapter 5.3.2). Furthermore, there was no change in CD86 expression on any of the DC subsets, indicating that $H$. polygyrus does not affect the activation of DCs. Further studies would be needed to characterize if and how DC phenotype and surface molecule expression are affected by $H$. polygyrus infection in this DBP-FITC model. I propose further experiments, to study differentiation of $\mathrm{T}$ cells (e.g. induction of Tregs) using sorted DCs after activation by DBP-FITC in an in vitro culture with $\mathrm{T}$ cells, which would help to determine if tolerogenic DCs are induced by $H$. polygyrus. ${ }^{127,}{ }^{128}$ To assess if DC migration from the skin to the draining LNs might be blocked by $H$. polygyrus and therefore affect initiation of the inflammatory reaction, as was the case in previous studies, migration markers such as chemokine receptor 7 (CCR7) on their surface and chemokine expression patterns in the skin and LN could be examined. ${ }^{219}$

Next, I analysed lymphocytes in the auricular LNs for cytokine expression, and for the proportions of Tregs and Th2 cells. My results indicated that $H$. polygyrus suppressed inflammation in the ear draining LNs during the peak of inflammation (day 7) as lymphocyte expansion was significantly decreased (chapter 5.3.3). Within $\mathrm{CD}^{+} \mathrm{T}$ cells, FoxP3 ${ }^{+}$Treg and $\mathrm{GATA}^{+}$Th2 cell proportions were significantly increased in the ear draining $\mathrm{LNs}$ after $H$. polygyrus infection, suggesting proportions of other subsets of T cells (such as Tbet $^{+}$Thl cells) might be decreased 
to compensate. As with the HDM model, further studies would be needed to demonstrate the importance of Tregs for the suppression of Th2 inflammation.

Finally, I analysed cytokine expression in the ear skin tissue via qPCR. TSLP has been demonstrated to be a key cytokine in the initiation of Th2-mediated inflammation in this model, and to be upregulated in patients with AD. ${ }^{213,214}$ While I found no profound expression of Tslp during the peak of DBP-FITC-induced inflammation, $H$. polygyrus-infected mice did seem to have decreased expression of this cytokine. My results suggest TSLP is only minimally present at day 7 after DBPFITC treatment (the peak of cellular inflammation), and an earlier time point (e.g. day 1 or day 2 ) would be more suitable to study the gene expression of Tslp and the effect of $H$. polygyrus on this cytokine. My results earlier demonstrated $H$. polygyrus increased IL- $4^{+} \mathrm{T}$ cells in the ear skin tissue, but did not significantly increase IL-13 production (chapter 3.3). I observed a considerable increase in Il4 and Ill3 gene expression after DBP-FITC treatment, and $H$. polygyrus caused a significant decrease in Ill3 expression (chapter 5.3.4). I detected no difference in Il4 expression. My results suggest that Ill3 gene expression could be more susceptible to the suppressive effects of $H$. polygyrus, than that of Il4. It would be interesting to investigate in which cells this decrease in Ill3 expression is occurring (e.g. Th2 cells or ILC2s). Notably, I detected there was a significant reduction of Foxp3 expression in $H$. polygyrus-infected mice compared to uninfected mice after DBP-FITC treatment, and expression of $I l 10$ and $\operatorname{Tgf} \beta$ was slightly, but not significantly, decreased. As $H$. polygyrus suppresses skin inflammation as seen by decreased ear thickness and total live immune cells in the ear draining LNs, it is possible an earlier time point might show an increased expression of these regulatory markers, and would require further experiments for validation. Investigation into alternative mechanisms for immune suppression by $H$. polygyrus could also be considered. 


\subsection{Future directions}

I propose further research will be needed to determine the effects of $H$. polygyrus on the ear skin tissue using the more physiologically relevant DBP-FITC model of AD. While ear thickness is reduced, it is not known what immune cells specifically are susceptible to the suppressive effects of $H$. polygyrus in the skin. Future studies I have planned using this model include analysis of T cells, including GATA ${ }^{+}$Th2 cells and FoxP3 $3^{+}$Tregs, in the ear skin tissue by histology, as Th2 cells in the skin tissue are a source of various Th2 cytokines, and essential in this model. ${ }^{215}$ The presence and function of innate cells including eosinophils, mast cells and ILC2s could also be analysed by histology by also looking at cytokine production or degranulation in the ear skin tissue.

Other regulatory cells are also induced by $H$. polygyrus infection. A population of regulatory $\mathrm{CD} 23^{\text {hi }} \mathrm{B}$ cells were induced in the guts after $H$. polygyrus infection, and were transferred to uninfected mice to suppress allergic airway inflammation. ${ }^{14}$ It would be interesting to investigate if this population is found in the auricular LNs of H. polygyrus-infected mice after DBP-FITC treatment.

AAMs were systemically induced in $H$. polygyrus-infected mice, and suppressed development of TID in NOD mice. ${ }^{113}$, 114 While the induction of AAMs by $H$. polygyrus have not been studied in the skin, results from the HDM model indicated they might be induced in the ear skin tissue after $H$. polygyrus-infection, making this population interesting to study further. I suggest analysis by qPCR and histology could provide more information on the presence and cytokine production by AAMs in the ear skin tissue after DBP-FITC treatment.

Research is ongoing in other laboratories into the molecular basis of helminthinduced suppression. To complement the studies done here using live helminth infection, I propose that experiments utilising the secreted molecules (HES) of $H$. polygyrus may allow further understanding of the mechanisms helminths use to downregulate immune responses. 


\subsection{Final conclusions}

My results outlined in this thesis demonstrate a potential therapeutic use for helminths, or their products, to suppress allergic diseases in the skin, and possibly inhibit the onset of the allergic march. Considering the ever-increasing burden of allergic diseases in New Zealand, and the wider world, this area of research is important and timely. ${ }^{220,221}$

To summarize, I have demonstrated:

1) H. polygyrus infection significantly increased the proportion of IL-4 producing $\mathrm{CD}^{+} \mathrm{T}$ cells in various tissues of mice, including the skin tissue.

2) Both allergy models of HDM-induced and DBP-FITC-induced Th2 inflammation were suppressed by $H$. polygyrus infection, characterized by significantly decreased ear thickness after DBP-FITC-induced CHS, and significantly decreased total live immune cell numbers in the auricular LNs in both allergy models.

3) This suppression by $H$. polygyrus infection coincided with an increased Treg proportion, and further experiments I have planned include depletion of Tregs to determine the importance of these regulatory cells in the suppression afforded by $H$. polygyrus.

4) H. polygyrus infection significantly decreased lymphocyte numbers (B cells, $\mathrm{CD}^{+} \mathrm{T}$ cells, and $\mathrm{CD}^{+} \mathrm{T}$ cells) in both allergy models, and increased GATA3 $^{+}$Th2 cell proportions in the auricular LNs in the DBP-FITC model. Furthermore, Ill3 expression was significantly decreased in the ear skin tissue during the peak of inflammation (day 7) after treatment with DBP-FITC. 


\section{Supplemental figures}


A
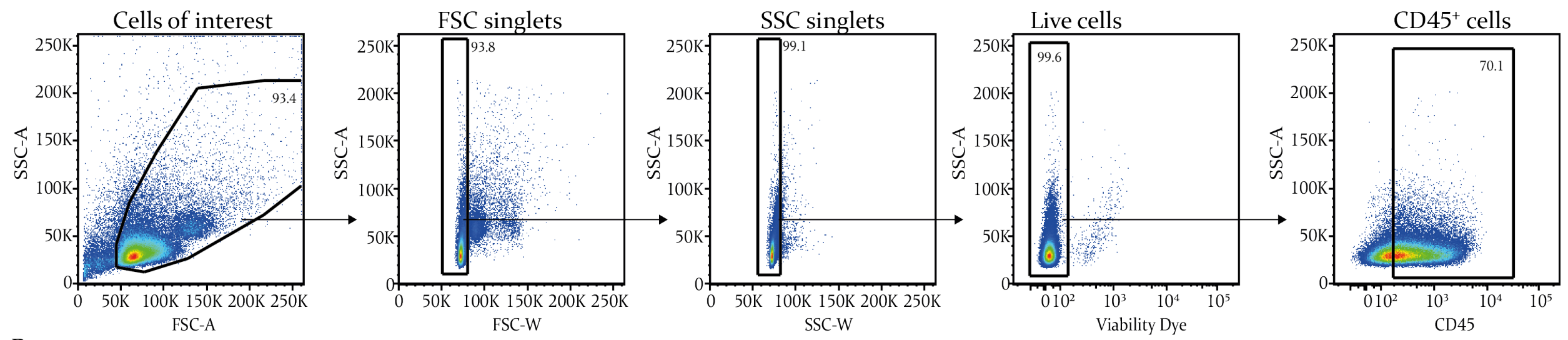

B
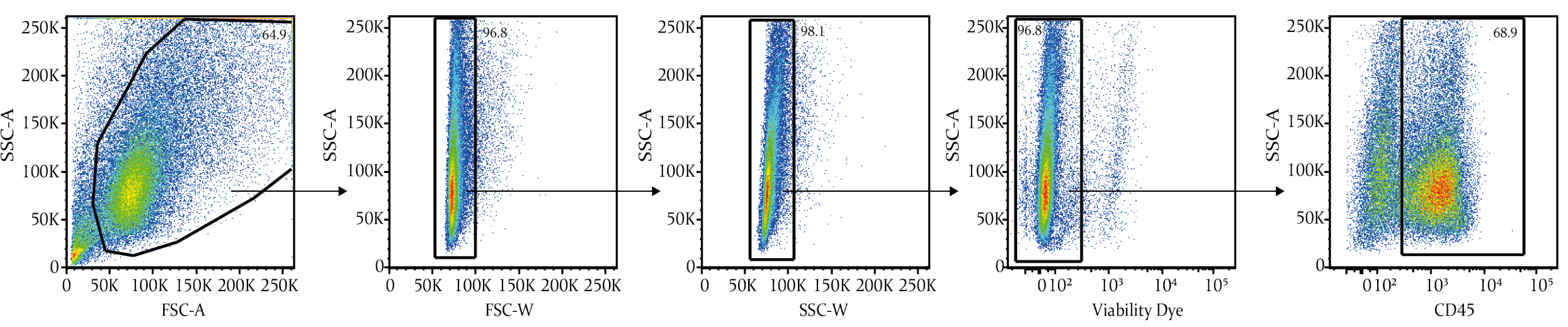

\section{Supplemental Figure 1: Gating strategy for LN and ear tissue}

Gating strategy used to identify $\mathrm{CD}^{+} 5^{+}$immune cells for cells isolated from A) $\mathrm{LN}$ or blood, and B) skin tissue or lung tissue 


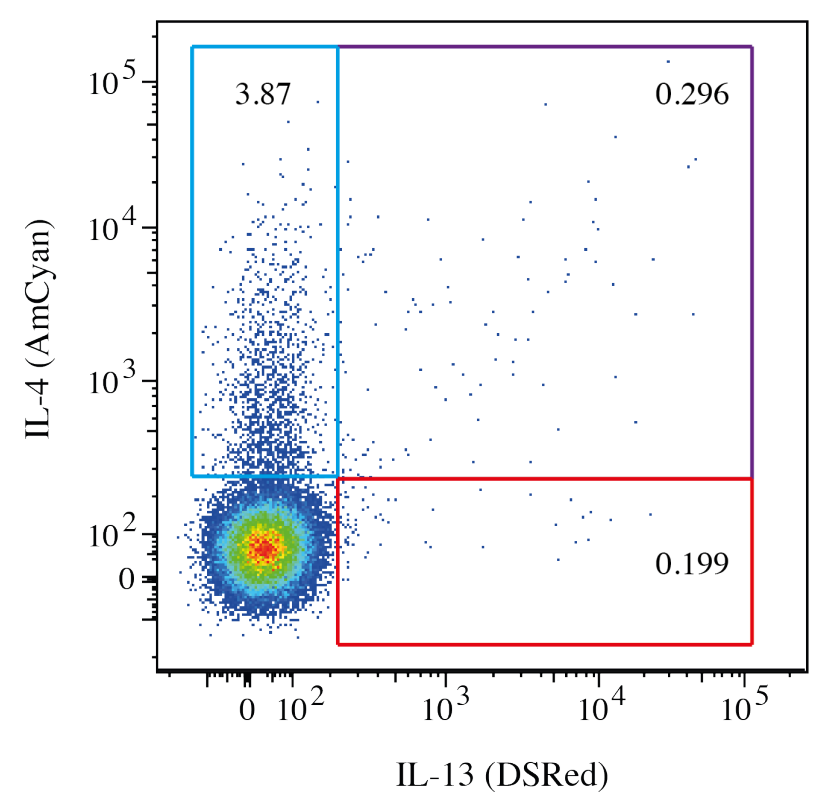

Supplemental Figure 2: Gating strategy for IL-4 and IL-13 expression in 4C13R reporter mice

Gating strategy used to identify IL-4 and IL-13 expression, by detection of the reporters AmCyan and DSRed respectively, in various immune cell populations. Blue gate: AmCyan ${ }^{+} \mathrm{IL}_{-} 4$ producing cells; red gate: DSRed ${ }^{+}$IL-13 producing cells; purple gate: IL-4 and IL-13 double-producing cells. 
References 
1. Hoeppli, R. The knowledge of parasites and parasitic infections from ancient times to the 17th century. Exp Parasitol 5, 398-419 (1956).

2. Strachan, D.P. Family size, infection and atopy: The first decade of the "hygiene hypothesis". Thorax 55, S2-10 (2000).

3. Greenwood, B.M. Autoimmune disease and parasitic infections in Nigerians. Lancet 2, 380-382 (1968).

4. Strachan, D.P. Hay fever, hygiene, and household size. BMJ 299, 1259-1260 (1989).

5. Heinrich, J., Popescu, M.A., Wjst, M., Goldstein, I.F. \& Wichmann, H.E. Atopy in children and parental social class. Am J Public Health 88, 1319-1324 (1998).

6. Von Mutius, E. et al. Skin test reactivity and number of siblings. BMJ 308, 692695 (1994).

7. Svanes, C., Jarvis, D., Chinn, S. \& Burney, P. Childhood environment and adult atopy: Results from the European Community Respiratory Health Survey. $J$ Allergy Clin Immunol 103, 415-420 (1999).

8. Alm, J.S., Swartz, J., Lilja, G., Scheynius, A. \& Pershagen, G. Atopy in children of families with an anthroposophic lifestyle. Lancet 353, 1485-1488 (1999).

9. Cooper, P.J. et al. Reduced risk of atopy among school-age children infected with geohelminth parasites in a rural area of the tropics. J Allergy Clin Immunol 111, 995-1000 (2003).

10. Araujo, M.I. et al. Inverse association between skin response to aeroallergens and Schistosoma mansoni infection. Int Arch Allergy Immunol 123, 145-148 (2000).

11. Van den Biggelaar, A.H.J. et al. Long-term treatment of intestinal helminths increases mite skin-test reactivity in Gabonese schoolchildren. J Infect Dis 189, 892-900 (2004).

12. Van den Biggelaar, A.H.J. et al. Decreased atopy in children infected with Schistosoma haematobium: A role for parasite-induced interleukin-10. Lancet 356, 1723-1727 (2000).

13. Wilson, M.S. et al. Suppression of allergic airway inflammation by helminthinduced regulatory T cells. J Exp Med 202, 1199-1212 (2005).

14. Wilson, M.S. et al. Helminth-induced CD19+CD23hi B cells modulate experimental allergic and autoimmune inflammation. Eur J Immunol 40, 16821696 (2010). 
15. Grainger, J.R. et al. Helminth secretions induce de novo T cell Foxp3 expression and regulatory function through the TGF $\beta$ pathway. J Exp Med 207, 2331-2341 (2010).

16. Finney, C.A.M., Taylor, M.D., Wilson, M.S. \& Maizels, R.M. Expansion and activation of CD4(+)CD25(+) regulatory T cells in Heligmosomoides polygyrus infection. Eur J Immunol 37, 1874-1886 (2007).

17. Daniłowicz-Luebert, E., O'Regan, N.L., Steinfelder, S. \& Hartmann, S. Modulation of specific and allergy-related immune responses by helminths. $J$ Biomed Biotechnol 2011 (2011).

18. Summers, R.W. Trichuris suis therapy in Crohn's disease. Gut 54, 87-90 (2005).

19. Croese, J. et al. A proof of concept study establishing Necator americanus in Crohn's patients and reservoir donors. Gut 55, 136-137 (2006).

20. Correale, J. \& Farez, M. Association between parasite infection and immune responses in multiple sclerosis. Ann Neurol 61, 97-108 (2007).

21. Feary, J.R. et al. Experimental hookworm infection: A randomized placebocontrolled trial in asthma. Clin Exp Allergy 40, 299-306 (2010).

22. Hotez, P.J. et al. Helminth infections: The great neglected tropical diseases. $J$ Clin Invest 118, 1311-1321 (2008).

23. Loukas, A. \& Prociv, P. Immune responses in hookworm infections. Clin Microbiol Rev 14, 689-703 (2001).

24. Allen, J.E. \& Maizels, R.M. Diversity and dialogue in immunity to helminths. Nat Rev Immunol 11, 375-388 (2011).

25. Hotez, P.J. et al. Hookworm infection. N Engl J Med 351, 799-807 (2004).

26. Stoltzfus, R.J., Dreyfuss, M.L., Chwaya, H.M. \& Albonico, M. Hookworm control as a strategy to prevent iron deficiency. Nutr Rev 55, 223-232 (1997).

27. Colley, D.G. \& Secor, W.E. Immunology of human schistosomiasis. Parasite Immunol 36, 347-357 (2014).

28. Butler, S.E. et al. Mechanism of anemia in Schistosoma mansoni-infected school children in Western Kenya. Am J Trop Med Hyg 87, 862-867 (2012).

29. Swai, B., Poggensee, G., Mtweve, S. \& Krantz, I. Female genital schistosomiasis as an evidence of a neglected cause for reproductive ill-health: A retrospective histopathological study from Tanzania. BMC Infect Dis 6, 51-58 (2006).

30. Van der Werf, M.J. et al. Quantification of clinical morbidity associated with schistosome infection in sub-Saharan Africa. Acta Tropica 86, 125-139 (2003). 
31. Resende Co, T., Hirsch, C.S., Toossi, Z., Dietze, R. \& Ribeiro-Rodrigues, R. Intestinal helminth co-infection has a negative impact on both antiMycobacterium tuberculosis immunity and clinical response to tuberculosis therapy. Clin Exp Immunol 147, 45-52 (2006).

32. Downs, J.A. et al. Urogenital schistosomiasis in women of reproductive age in Tanzania's Lake Victoria region. Am J Trop Med Hyg 84, 364-369 (2011).

33. Kjetland, E.F. et al. Association between genital schistosomiasis and HIV in rural Zimbabwean women. AIDS 20, 593-600 (2006).

34. Degarege, A., Legesse, M., Medhin, G., Animut, A. \& Erko, B. Malaria and related outcomes in patients with intestinal helminths: A cross-sectional study. BMC Infect Dis 12, 291 (2012).

35. Le Hesran, J.-Y. et al. Severe malaria attack is associated with high prevalence of Ascaris lumbricoides infection among children in rural Senegal. T Roy Soc Trop Med H 98, 397-399 (2004).

36. Lyke, K.E. et al. Association of Schistosoma haematobium infection with protection against acute Plasmodium falciparum malaria in Malian children. Am J Trop Med Hyg 73, 1124-1130 (2005).

37. Elliot, A.M. et al. Effects of maternal and infant co-infections, and of maternal immunisation, on the infant response to BCG and tetanus immunisation. Vaccine 29, 247-255 (2010).

38. Osborne, L.C. et al. Virus-helminth coinfection reveals a microbiotaindependent mechanism of immunomodulation. Science 345, 578-582 (2014).

39. Reese, T.A. et al. Helminth infection reactivates latent $\gamma$-herpesvirus via cytokine competition at a viral promoter. Science 345, 573-577 (2014).

40. Mosmann, T.R., Cherwinski, H., Bond, M.W., Giedlin, M.A. \& Coffman, R.L. Two types of murine helper $\mathrm{T}$ cell clone. I. Definition according to profiles of lymphokine activities and secreted proteins. J Immunol 136, 2348-2357 (1986).

41. Raphael, I., Nalawade, S., Eagar, T.N. \& Forsthuber, T.G. T cell subsets and their signature cytokines in autoimmune and inflammatory diseases. Cytokine 74, 517 (2015).

42. Berger, A. Th1 and Th2 responses: What are they? BMJ 321, 424-424 (2000).

43. Park, H. et al. A distinct lineage of CD4 T cells regulates tissue inflammation by producing interleukin-17. Nat Immunol 6, 1133-1141 (2005).

44. Gause, W.C., Wynn, T.A. \& Allen, J.E. Type 2 immunity and wound healing: Evolutionary refinement of adaptive immunity by helminths. Nat Rev Immunol 13, 607-614 (2013). 
45. Tan, C. \& Gery, I. The unique features of Th9 cells and their products. Crit Rev Immunol 32, 1-10 (2012).

46. Khan, W.I. et al. Modulation of intestinal muscle contraction by interleukin-9 (IL-9) or IL-9 neutralization: Correlation with worm expulsion in murine nematode infections. Infec Immun 71, 2430-2438 (2003).

47. Forbes, E.E. et al. IL-9- and mast cell-mediated intestinal permeability predisposes to oral antigen hypersensitivity. J Exp Med 205, 897-913 (2008).

48. Vignali, D.A.A., Collison, L.W. \& Workman, C.J. How regulatory T cells work. Nat Rev Immunol 8, 523-532 (2008).

49. Sakaguchi, S., Sakaguchi, N., Asano, M., Itoh, M. \& Toda, M. Immunologic selftolerance maintained by activated $\mathrm{T}$ cells expressing IL-2 receptor alphachains (CD25). Breakdown of a single mechanism of self-tolerance causes various autoimmune diseases. J Immunol 155, 1151-1164 (1995).

50. Röcken, M., Racke, M. \& Shevach, E.M. IL-4-induced immune deviation as antigen-specific therapy for inflammatory autoimmune disease. Immunol Today 17, 225-231 (1996).

51. Allen, J.E. \& Wynn, T.A. Evolution of Th2 immunity: A rapid repair response to tissue destructive pathogens. PLoS Pathog 7, e1002003-1002004 (2011).

52. Schmitz, J. et al. IL-33, an interleukin-1-like cytokine that signals via the IL-1 receptor-related protein ST2 and induces T helper type 2-associated cytokines. Immunity 23, 479-490 (2005).

53. Zhou, B. et al. Thymic stromal lymphopoietin as a key initiator of allergic airway inflammation in mice. Nat Immunol 6, 1047-1053 (2005).

54. Paul, W.E. \& Zhu, J. How are T(H)2-type immune responses initiated and amplified? Nat Rev Immunol 10, 225-235 (2010).

55. Massacand, J.C. et al. Helminth products bypass the need for TSLP in Th2 immune responses by directly modulating dendritic cell function. Proc Natl Acad Sci USA 106, 13968-13973 (2009).

56. Anderson, E.L. et al. IL-33 mediates reactive eosinophilopoiesis in response to airborne allergen exposure. Allergy 71, 977-988 (2016).

57. Von Moltke, J., Ji, M., Liang, H.-E. \& Locksley, R.M. Tuft-cell-derived IL-25 regulates an intestinal ILC2-epithelial response circuit. Nature 529, 221-225 (2016).

58. Allakhverdi, Z., Smith, D.E., Comeau, M.R. \& Delespesse, G. Cutting edge: The ST2 ligand IL-33 potently activates and drives maturation of human mast cells. J Immunol 179, 2051-2054 (2007). 
59. Zheng, W.-P. \& Flavell, R.A. The transcription factor GATA-3 is necessary and sufficient for Th2 cytokine gene expression in CD4 T cells. Cell 89, 587-596 (1997).

60. Kaplan, M.H., Schindler, U., Smiley, S.T. \& Grusby, M.J. STAT6 is required for mediating responses to IL-4 and for development of Th2 cells. Immunity 4, 313-319 (1996).

61. Stone, K.D., Prussin, C. \& Metcalfe, D.D. IgE, mast cells, basophils, and eosinophils. J Allergy Clin Immunol 125, S73-80 (2010).

62. Kato, A., Favoreto, S., Avila, P.C. \& Schleimer, R.P. TLR3- and Th2 cytokinedependent production of thymic stromal lymphopoietin in human airway epithelial cells. J Immunol 179, 1080-1087 (2007).

63. Yang, M. et al. Eotaxin-2 and IL-5 cooperate in the lung to regulate IL-13 production and airway eosinophilia and hyperreactivity. Journal of Allergy and Clinical Immunology 112, 935-943 (2003).

64. Townsend, J.M. et al. IL-9-deficient mice establish fundamental roles for IL-9 in pulmonary mastocytosis and goblet cell hyperplasia but not $\mathrm{T}$ cell development. Immunity 13, 573-583 (2000).

65. Urban, J.F., Katona, I.M. \& Finkelman, F.D. Heligmosomoides polygyrus: CD4+ but not CD8+ T cells regulate the IgE response and protective immunity in mice. Exp Parasitol 73, 500-511 (1991).

66. Urban, J.F., Katona, I.M., Paul, W.E. \& Finkelman, F.D. Interleukin 4 is important in protective immunity to a gastrointestinal nematode infection in mice. Proceedings of the National Academy of Sciences of the United States of America 88, 5513-5517 (1991).

67. Urban, J.F., Noben-Trauth, N., Schopf, L., Madden, K.B. \& Finkelman, F.D. Cutting edge: IL-4 receptor expression by non-bone marrow-derived cells is required to expel gastrointestinal nematode parasites. J Immunol 167, 60786081 (2001).

68. Greenwald, R.J. et al. Effects of blocking B7-1 and B7-2 interactions during a type 2 in vivo immune response. J Immunol 158, 4088-4096 (1997).

69. Phythian-Adams, A.T. et al. CD11c depletion severely disrupts Th2 induction and development in vivo. J Exp Med 207, 2089-2096 (2010).

70. Yoshimoto, T. et al. Basophils contribute to TH2-IgE responses in vivo via IL-4 production and presentation of peptide-MHC class II complexes to CD4+ T cells. Nat Immunol 10, 706-712 (2009).

71. Ohnmacht, C. \& Voehringer, D. Basophil effector function and homeostasis during helminth infection. Blood 113, 2816-2825 (2009). 
72. Sokol, C.L. et al. Basophils function as antigen-presenting cells for an allergeninduced T helper type 2 response. Nat Immunol 10, 713-721 (2009).

73. Smith, K.A. et al. Type 2 innate immunity in helminth infection is induced redundantly and acts autonomously following CD11c+ cell depletion. Infect Immun 80, 3481-3489 (2012).

74. Neill, D.R. et al. Nuocytes represent a new innate effector leukocyte that mediates type-2 immunity. Nature 464, 1367-1370 (2010).

75. Pelly, V.S. et al. IL-4-producing ILC2s are required for the differentiation of TH2 cells following Heligmosomoides polygyrus infection. Mucosal Immunol 9, 1407-1417 (2016).

76. Rosenberg, H.F., Dyer, K.D. \& Foster, P.S. Eosinophils: Changing perspectives in health and disease. Nat Rev Immunol 13, 9-22 (2012).

77. Anthony, R.M., Rutitzky, L.I., Urban, J.F., Stadecker, M.J. \& Gause, W.C. Protective immune mechanisms in helminth infection. Nat Rev Immunol 7, 975-987 (2007).

78. Anthony, R.M. et al. Memory TH2 cells induce alternatively activated macrophages to mediate protection against nematode parasites. Nat Med 12, 955-960 (2006).

79. Reynolds, L.A., Filbey, K.J. \& Maizels, R.M. Immunity to the model intestinal helminth parasite Heligmosomoides polygyrus. Semin Immunopathol 34, 829846 (2012).

80. McDermott, J.R. et al. Mast cells disrupt epithelial barrier function during enteric nematode infection. Proc Natl Acad Sci USA 100, 7761-7766 (2003).

81. Liu, Q. et al. B cells have distinct roles in host protection against different nematode parasites. J Immunol 184, 5213-5223 (2010).

82. Miyake, K., Tanaka, T. \& McNeil, P.L. Disruption-induced mucus secretion: Repair and protection. PLoS Biol 4, e276-277 (2006).

83. Zeinoun, T., Aftimos, G., Bou Saba, S. \& Nammour, S. Eosinophils and mastocytes in healing laser excision wounds. Lasers Med Sci 24, 307-312 (2008).

84. Nishikori, Y., Shiota, N. \& Okunishi, H. The role of mast cells in cutaneous wound healing in streptozotocin-induced diabetic mice. Arch Dermatol Res 306, 823-835 (2014).

85. Pesce, J.T. et al. Arginase-1-expressing macrophages suppress Th2 cytokinedriven inflammation and fibrosis. PLoS Pathog 5, e1000371-1000315 (2009). 
86. Ramalingam, T.R. \& Wynn, T.A. Mechanisms of fibrosis: Therapeutic translation for fibrotic disease. Nat Med 18, 1028-1040 (2012).

87. Monticelli, L.A. et al. Innate lymphoid cells promote lung-tissue homeostasis after infection with influenza virus. Nat Immunol 12, 1045-1054 (2011).

88. Oh, M.H. et al. IL-13 induces skin fibrosis in atopic dermatitis by thymic stromal lymphopoietin. J Immunol 186, 7232-7242 (2011).

89. Clark, W.C. Origins of the parasitic habit in the nematoda. Int J Parasitol 24, 1117-1129 (1994).

90. Anderson, R.C. Why do fish have so few roundworm (nematode) parasites? Environ Biol Fish 46, 1-5 (1996).

91. Gouÿ de Bellocq, J. et al. Phylogeny of the Trichostrongylina (nematoda) inferred from 28S rDNA sequences. Mol Phylogenet Evol 19, 430-442 (2001).

92. Filbey, K.J. et al. Innate and adaptive type 2 immune cell responses in genetically controlled resistance to intestinal helminth infection. Immunol Cell Biol 92, 436-448 (2014).

93. McSorley, H.J. et al. Suppression of type 2 immunity and allergic airway inflammation by secreted products of the helminth Heligmosomoides polygyrus. Eur J Immunol 42, 2667-2682 (2012).

94. Hewitson, J.P. et al. Proteomic analysis of secretory products from the model gastrointestinal nematode Heligmosomoides polygyrus reveals dominance of Venom Allergen-Like (VAL) proteins. J Proteomics 74, 1573-1594 (2011).

95. Moreno, Y. et al. Proteomic analysis of excretory-secretory products of Heligmosomoides polygyrus assessed with next-generation sequencing transcriptomic information. PLoS Negl Trop Dis 5, e1370-1311 (2011).

96. Gondek, D.C., Lu, L.F., Quezada, S.A., Sakaguchi, S. \& Noelle, R.J. Cutting edge: Contact-mediated suppression by CD4+CD25+ regulatory cells involves a granzyme B-dependent, perforin-independent mechanism. J Immunol 174, 1783-1786 (2005).

97. Pandiyan, P., Zheng, L., Ishihara, S., Reed, J. \& Lenardo, M.J. CD4+CD25+Foxp3+ regulatory $\mathrm{T}$ cells induce cytokine deprivation-mediated apoptosis of effector CD4+ T cells. Nat Immunol 8, 1353-1362 (2007).

98. Akdis, C.A. \& Akdis, M. Mechanisms and treatment of allergic disease in the big picture of regulatory T cells. J Allergy Clin Immunol 123, 735-746 (2009).

99. Zhang, H. et al. Subsets of regulatory T cells and their roles in allergy. J Transl Med 12, 125 (2014). 
100. Cederbom, L., Hall, H. \& Ivars, F. CD4+CD25+ regulatory T cells down-regulate co-stimulatory molecules on antigen-presenting cells. Eur J Immunol 30, 15381543 (2000).

101. Joetham, A. et al. Naturally occurring lung CD4+CD25+ $\mathrm{T}$ cell regulation of airway allergic responses depends on IL-10 induction of TGF $\beta$. J Immunol 178, 1433-1442 (2007).

102. Asseman, C., Mauze, S., Leach, M.W., Coffman, R.L. \& Powrie, F. An essential role for interleukin 10 in the function of regulatory $\mathrm{T}$ cells that inhibit intestinal inflammation. The Journal of Experimental Medicine 190, 995-1004 (1999).

103. Li, M.O., Wan, Y.Y. \& Flavell, R.A. T cell-produced transforming growth factor$\beta 1$ controls $\mathrm{T}$ cell tolerance and regulates Th1- and Th17-cell differentiation. Immunity 26, 579-591 (2007).

104. Nakamura, K., Kitani, A. \& Strober, W. Cell contact-dependent immunosuppression by $\mathrm{CD} 4(+) \mathrm{CD} 25(+)$ regulatory $\mathrm{T}$ cells is mediated by cell surface-bound transforming growth factor beta. J Exp Med 194, 629-644 (2001).

105. Smith, K.A. et al. Low-level regulatory T-cell activity is essential for functional type-2 effector immunity to expel gastrointestinal helminths. Mucosal Immunol 9, 428-443 (2016).

106. Maizels, R.M. et al. Immune modulation and modulators in Heligmosomoides polygyrus infection. Exp Parasitol 132, 76-89 (2012).

107. Rausch, S. et al. Establishment of nematode infection despite increased Th2 responses and immunopathology after selective depletion of Foxp3+ cells. Eur J Immunol 39, 3066-3077 (2009).

108. Blair, P.A. et al. CD19+CD24hiCD38hi B cells exhibit regulatory capacity in healthy individuals but are functionally impaired in systemic lupus erythematosus patients. Immunity 32, 129-140 (2010).

109. Hussaarts, L., van der Vlugt, L.E.P.M., Yazdanbakhsh, M. \& Smits, H.H. Regulatory B-cell induction by helminths: Implications for allergic disease. $J$ Allergy Clin Immunol 128, 733-739 (2011).

110. Broadhurst, M.J. et al. Upregulation of retinal dehydrogenase 2 in alternatively activated macrophages during retinoid-dependent type-2 immunity to helminth infection in mice. PLoS Pathog 8, e1002883-1002814 (2012).

111. Terrazas, L.I., Montero, D., Terrazas, C.A., Reyes, J.L. \& Rodríguez-Sosa, M. Role of the Programmed Death-1 pathway in the suppressive activity of alternatively activated macrophages in experimental cysticercosis. Int $J$ Parasitol 35, 1349-1358 (2005). 
112. Gordon, S. \& Martinez, F.O. Alternative activation of macrophages: Mechanism and functions. Immunity 32, 593-604 (2010).

113. Weng, M. et al. Alternatively activated macrophages in intestinal helminth infection: Effects on concurrent bacterial colitis. J Immunol 179, 4721-4731 (2007).

114. Liu, Q. et al. Helminth infection can reduce insulitis and type 1 diabetes through CD25- and IL-10-independent mechanisms. Infect Immun 77, 53475358 (2009).

115. Groux, H. et al. A CD4+ T-cell subset inhibits antigen-specific T-cell responses and prevents colitis. Nature 389, 737-742 (1997).

116. Castillo, P. \& Kolls, J.K. IL-10: A paradigm for counterregulatory cytokines. J Immunol 197, 1529-1530 (2016).

117. Fiorentino, D.F. et al. IL-10 acts on the antigen-presenting cell to inhibit cytokine production by Th1 cells. J Immunol 146, 3444-3451 (1991).

118. Fiorentino, D.F., Zlotnik, A., Mosmann, T.R., Howard, M. \& OGarra, A. IL-10 inhibits cytokine production by activated macrophages. J Immunol 147, 38153822 (1991).

119. Elliott, D.E. et al. Heligmosomoides polygyrus inhibits established colitis in IL10-deficient mice. Eur J Immunol 34, 2690-2698 (2004).

120. Bashir, M.E.H., Andersen, P., Fuss, I.J., Shi, H.N. \& Nagler-Anderson, C. An enteric helminth infection protects against an allergic response to dietary antigen. J Immunol 169, 3284-3292 (2002).

121. Chen, W. \& ten Dijke, P. Immunoregulation by members of the TGF $\beta$ superfamily. Nat Rev Immunol 16, 723-740 (2016).

122. Doligalska, M., Rzepecka, J., Drela, N., Donskow, K. \& Gerwel-Wronka, M. The role of TGF $\beta$ in mice infected with Heligmosomoides polygyrus. Parasite Immunol 28, 387-395 (2006).

123. McSorley, H.J., Blair, N.F., Smith, K.A., McKenzie, A.N.J. \& Maizels, R.M. Blockade of IL-33 release and suppression of type 2 innate lymphoid cell responses by helminth secreted products in airway allergy. Mucosal Immunol 7, 1068-1078 (2014).

124. Maizels, R.M. \& McSorley, H.J. Regulation of the host immune system by helminth parasites. J Allergy Clin Immunol 138, 666-675 (2016).

125. McSorley, H.J., Hewitson, J.P. \& Maizels, R.M. Immunomodulation by helminth parasites: Defining mechanisms and mediators. Int J Parasitol 43, 301-310 (2013). 
126. Jackson, J.A., Friberg, I.M., Little, S. \& Bradley, J.E. Review series on helminths, immune modulation and the hygiene hypothesis: Immunity against helminths and immunological phenomena in modern human populations: Coevolutionary legacies? Immunology 126, 18-27 (2009).

127. Li, Z. et al. The phenotype and function of naturally existing regulatory dendritic cells in nematode-infected mice. Int J Parasitol 41, 1129-1137 (2011).

128. Smith, K.A. et al. Chronic helminth infection promotes immune regulation in vivo through dominance of CD11cloCD103- dendritic cells. J Immunol 186, 7098-7109 (2011).

129. Bando, J.K. \& Colonna, M. Innate lymphoid cell function in the context of adaptive immunity. Nat Immunol 17, 783-789 (2016).

130. Klose, C.S.N. \& Artis, D. Innate lymphoid cells as regulators of immunity, inflammation and tissue homeostasis. Nat Immunol 17, 765-774 (2016).

131. Théry, C. Exosomes: Secreted vesicles and intercellular communications. F1000 Biol Rep 3, 15 (2011).

132. Buck, A.H. et al. Exosomes secreted by nematode parasites transfer small RNAs to mammalian cells and modulate innate immunity. Nat Commun 5, 5488 (2014).

133. Coakley, G., Maizels, R.M. \& Buck, A.H. Exosomes and other extracellular vesicles: The new communicators in parasite infections. Trends Parasitol 31, 477-489 (2015).

134. Chung, H. et al. Gut immune maturation depends on colonization with a hostspecific microbiota. Cell 149, 1578-1593 (2012).

135. Ramanan, D. et al. Helminth infection promotes colonization resistance via type 2 immunity. Science 352, 608-612 (2016).

136. Loke, P. \& Lim, Y.A.L. Helminths and the microbiota: Parts of the hygiene hypothesis. Parasite Immunol 37, 314-323 (2015).

137. Reynolds, L.A. et al. Commensal-pathogen interactions in the intestinal tract: Lactobacilli promote infection with, and are promoted by, helminth parasites. Gut Microbes 5, 522-532 (2014).

138. Zaiss, M.M. et al. The intestinal microbiota contributes to the ability of helminths to modulate allergic inflammation. Immunity 43, 998-1010 (2015).

139. Coakley, G., Buck, A.H. \& Maizels, R.M. Host parasite communicationsMessages from helminths for the immune system: Parasite communication and cell-cell interactions. Mol Biochem Parasitol 208, 33-40 (2016). 
140. Nestle, F.O., Di Meglio, P., Qin, J.-Z. \& Nickoloff, B.J. Skin immune sentinels in health and disease. Nat Rev Immunol 9, 679-691 (2009).

141. Lebre, M.C. et al. Human keratinocytes express functional Toll-like receptor 3, 4, 5, and 9. J Invest Dermatol 127, 331-341 (2007).

142. Feldmeyer, L. et al. The inflammasome mediates UVB-induced activation and secretion of interleukin-1beta by keratinocytes. Curr Biol 17, 1140-1145 (2007).

143. Ochiai, S. et al. CD326(lo)CD103(lo)CD11b(lo) dermal dendritic cells are activated by thymic stromal lymphopoietin during contact sensitization in mice. J Immunol 193, 2504-2511 (2014).

144. Mantovani, A., Biswas, S.K., Galdiero, M.R., Sica, A. \& Locati, M. Macrophage plasticity and polarization in tissue repair and remodelling. J Pathol 229, 176185 (2012).

145. Otsuka, A., Nonomura, Y. \& Kabashima, K. Roles of basophils and mast cells in cutaneous inflammation. Semin Immunopathol 38, 563-570 (2016).

146. Hershko, A.Y. et al. Mast cell interleukin-2 production contributes to suppression of chronic allergic dermatitis. Immunity 35, 562-571 (2011).

147. Morita, H. et al. An interleukin-33-mast cell-interleukin-2 axis suppresses papain-induced allergic inflammation by promoting regulatory $\mathrm{T}$ cell numbers. Immunity 43, 175-186 (2015).

148. Grimbaldeston, M.A., Nakae, S., Kalesnikoff, J., Tsai, M. \& Galli, S.J. Mast cellderived interleukin-10 limits skin pathology in contact dermatitis and chronic irradiation with ultraviolet B. Nat Immunol 8, 1095-1104 (2007).

149. Roediger, B. et al. Cutaneous immunosurveillance and regulation of inflammation by group 2 innate lymphoid cells. Nat Immunol 14, 564-573 (2013).

150. Bos, J.D. et al. The skin immune system (SIS): Distribution and immunophenotype of lymphocyte subpopulations in normal human skin. $J$ Invest Dermatol 88, 569-573 (1987).

151. Pasparakis, M., Haase, I. \& Nestle, F.O. Mechanisms regulating skin immunity and inflammation. Nat Rev Immunol 14, 289-301 (2014).

152. Weidinger, S. \& Novak, N. Atopic dermatitis. Lancet 387, 1109-1122 (2016).

153. Kawakami, T., Ando, T., Kimura, M., Wilson, B.S. \& Kawakami, Y. Mast cells in atopic dermatitis. Curr Opin Immunol 21, 666-678 (2009). 
154. Deckers, I.A.G. et al. Investigating international time trends in the incidence and prevalence of atopic eczema 1990-2010: A systematic review of epidemiological studies. PLoS ONE 7, e39803-39828 (2012).

155. Weidinger, S. et al. Loss-of-function variations within the filaggrin gene predispose for atopic dermatitis with allergic sensitizations. Journal of Allergy and Clinical Immunology 118, 214-219 (2006).

156. Palmer, C.N.A. et al. Common loss-of-function variants of the epidermal barrier protein filaggrin are a major predisposing factor for atopic dermatitis. Nat Genet 38, 441-446 (2006).

157. Hammad, H. \& Lambrecht, B.N. Barrier epithelial cells and the control of type 2 immunity. Immunity 43, 29-40 (2015).

158. Peine, M., Marek, R.M. \& Löhning, M. IL-33 in T Cell differentiation, function, and immune homeostasis. Trends Immunol 37, 321-333 (2016).

159. Roediger, B., Kyle, R., Le Gros, G. \& Weninger, W. Dermal group 2 innate lymphoid cells in atopic dermatitis and allergy. Curr Opin Immunol 31, 108114 (2014).

160. Fyhrquist, N. et al. Foxp3+ cells control Th2 responses in a murine model of atopic dermatitis. J Invest Dermatol 132, 1672-1680 (2012).

161. Jin, H., He, R., Oyoshi, M. \& Geha, R.S. Animal models of atopic dermatitis. J Invest Dermatol 129, 31-40 (2009).

162. Shigeno, T., Katakuse, M., Fujita, T., Mukoyama, Y. \& Watanabe, H. Phthalate ester-induced thymic stromal lymphopoietin mediates allergic dermatitis in mice. Immunology 128, e849-857 (2009).

163. Li, M. et al. Topical vitamin D3 and low-calcemic analogs induce thymic stromal lymphopoietin in mouse keratinocytes and trigger an atopic dermatitis. Proc Natl Acad Sci USA 103, 11736-11741 (2006).

164. Li, M. et al. Induction of thymic stromal lymphopoietin expression in keratinocytes is necessary for generating an atopic dermatitis upon application of the active vitamin D3 analogue MC903 on mouse skin. J Invest Dermatol 129, 498-502 (2009).

165. Paternoster, L. et al. Meta-analysis of genome-wide association studies identifies three new risk loci for atopic dermatitis. Nat Genet 44, 187-192 (2011).

166. Zhao, Y., Bao, L., Chan, L.S., DiPietro, L.A. \& Chen, L. Aberrant wound healing in an epidermal interleukin-4 transgenic mouse model of atopic dermatitis. PLoS ONE 11, e0146451 (2016). 
167. Yoo, J. et al. Spontaneous atopic dermatitis in mice expressing an inducible thymic stromal lymphopoietin transgene specifically in the skin. J Exp Med 202, 541-549 (2005).

168. Lane, P.W. Two new mutations in linkage group XVI of the house mouse. Flaky tail and varitint-waddler-J. J Hered 63, 135-140 (1972).

169. Knol, E.F. \& Hijnen, D. Atopic dermatitis: A tale of two distinct pathomechanisms that make you itch. Eur J Immunol 46, 2512-2515 (2016).

170. Matsuda, H. et al. Development of atopic dermatitis-like skin lesion with IgE hyperproduction in NC/Nga mice. Int Immunol 9, 461-466 (1997).

171. Vestergaard, C. et al. Overproduction of Th2-specific chemokines in NC/Nga mice exhibiting atopic dermatitis-like lesions. J Clin Invest 104, 1097-1105 (1999).

172. Dharmage, S.C. et al. Atopic dermatitis and the atopic march revisited. Allergy 69, 17-27 (2014).

173. Hogan, M.B., Peele, K. \& Wilson, N.W. Skin barrier function and its importance at the start of the atopic march. J Allergy 2012, 1-7 (2012).

174. Leyva-Castillo, J.M., Hener, P., Jiang, H. \& Li, M. TSLP produced by keratinocytes promotes allergen sensitization through skin and thereby triggers atopic march in mice. J Invest Dermatol 133, 154-163 (2013).

175. Saunders, S.P. et al. Spontaneous atopic dermatitis is mediated by innate immunity, with the secondary lung inflammation of the atopic march requiring adaptive immunity. J Allergy Clin Immunol 137, 482-491 (2016).

176. Hartmann, S. et al. Gastrointestinal nematode infection interferes with experimental allergic airway inflammation but not atopic dermatitis. Clin Exp Allergy 39, 1585-1596 (2009).

177. Mpairwe, H. et al. Anthelminthic treatment during pregnancy is associated with increased risk of infantile eczema: Randomised-controlled trial results. Pediatr Allergy Immunol 22, 305-312 (2011).

178. Camberis, M., Le Gros, G. \& Urban, J. Animal model of Nippostrongylus brasiliensis and Heligmosomoides polygyrus. Curr Protoc Immunol Chapter 19, Unit 19.12.11-19.12.27 (2003).

179. Mohrs, K., Harris, D.P., Lund, F.E. \& Mohrs, M. Systemic dissemination and persistence of Th2 and type 2 cells in response to infection with a strictly enteric nematode parasite. J Immunol 175, 5306-5313 (2005).

180. Elbe-Bürger, A. et al. Overexpression of IL-4 alters the homeostasis in the skin. J Invest Dermatol 118, 767-778 (2002). 
181. Dalessandri, T., Crawford, G., Hayes, M., Castro Seoane, R. \& Strid, J. IL-13 from intraepithelial lymphocytes regulates tissue homeostasis and protects against carcinogenesis in the skin. Nat Commun 7, 12080 (2016).

182. Huang, Y. et al. IL-25-responsive, lineage-negative KLRG1hi cells are multipotential 'inflammatory' type 2 innate lymphoid cells. Nat Immunol 16, 161-169 (2014).

183. Loke, P. et al. Alternative activation is an innate response to injury that requires CD4+ T cells to be sustained during chronic infection. J Immunol 179, 3926-3936 (2007).

184. Eming, S.A. et al. Accelerated wound closure in mice deficient for interleukin10. Am J Pathol 170, 188-202 (2010).

185. Zock, J.P. et al. Distribution and determinants of house dust mite allergens in Europe: The European Community Respiratory Health Survey II. J Allergy Clin Immunol 118, 682-690 (2006).

186. Jacquet, A. Innate immune responses in house dust mite allergy. ISRN Allergy 2013, 735031 (2013).

187. Bessot, J.C. \& Pauli, G. Mite allergens: An overview. Eur Ann Allergy Clin Immunol 43, 141-156 (2011).

188. Wan, H. et al. Quantitative structural and biochemical analyses of tight junction dynamics following exposure of epithelial cells to house dust mite allergen Der p 1. Clin Exp Allergy 30, 685-698 (2000).

189. Douwes, J. et al. (1-->3)-beta-D-glucan and endotoxin in house dust and peak flow variability in children. Am J Respir Crit Care Med 162, 1348-1354 (2000).

190. Hammad, H. et al. House dust mite allergen induces asthma via Toll-like receptor 4 triggering of airway structural cells. Nat Med 15, 410-416 (2009).

191. Chiou, Y.L. \& Lin, C.Y. Der p 2 activates airway smooth muscle cells in a TLR2/MyD88-dependent manner to induce an inflammatory response. J Cell Physiol 220, 311-318 (2009).

192. Barrett, N.A. et al. Dectin-2 mediates Th2 immunity through the generation of cysteinyl leukotrienes. J Exp Med 208, 593-604 (2011).

193. Dai, X. et al. Mite allergen is a danger signal for the skin via activation of inflammasome in keratinocytes. J Allergy Clin Immunol 127, 806-814 (2011).

194. Eisenbarth, S.C. et al. Lipopolysaccharide-enhanced, Toll-like Receptor 4dependent T Helper Cell Type 2 Responses to Inhaled Antigen. J Exp Med 196, 1645-1651 (2002). 
195. Arshad, S.H. Does Exposure to Indoor Allergens Contribute to the Development of Asthma and Allergy? Current allergy and asthma reports 10, 49-55 (2009).

196. Hammad, H. et al. Inflammatory dendritic cells-not basophils-are necessary and sufficient for induction of Th2 immunity to inhaled house dust mite allergen. J Exp Med 207, 2097-2111 (2010).

197. Zoltowska, A.M. et al. The interleukin-33 receptor ST2 is important for the development of peripheral airway hyperresponsiveness and inflammation in a house dust mite mouse model of asthma. Clin Exp Allergy 46, 479-490 (2016).

198. Noble, A. \& Zhao, J. Follicular helper T cells are responsible for IgE responses to Der p 1 following house dust mite sensitization in mice. Clin Exp Allergy 46, 1075-1082 (2016).

199. Guo, L. et al. Innate immunological function of TH2 cells in vivo. Nat Immunol 16, 1051-1059 (2015).

200. Camberis, M. et al. Evaluating the in vivo Th2 priming potential among common allergens. J Immunol Methods 394, 62-72 (2013).

201. Friedmann, P.S. The role of dust mite antigen sensitization and atopic dermatitis. Clin Exp Allergy 29, 869-872 (1999).

202. Nakamura, T. et al. Reduction of skin barrier function by proteolytic activity of a recombinant house dust mite major allergen Der f 1 . J Invest Dermatol 126, 2719-2723 (2006).

203. Jeong, S.K. et al. Mite and cockroach allergens activate protease- activated receptor 2 and delay epidermal permeability barrier recovery. J Invest Dermatol 128, 1930-1939 (2008).

204. Tyagi, N. et al. Comparisons of allergenic and metazoan parasite proteins: Allergy the price of immunity. PLoS Comput Biol 11, e1004546 (2015).

205. Verhagen, J. et al. Absence of T-regulatory cell expression and function in atopic dermatitis skin. Journal of Allergy and Clinical Immunology 117, 176183 (2006).

206. Matsumoto, K., Mizukoshi, K., Oyobikawa, M., Ohshima, H. \& Tagami, H. Establishment of an atopic dermatitis-like skin model in a hairless mouse by repeated elicitation of contact hypersensitivity that enables to conduct functional analyses of the stratum corneum with various non-invasive biophysical instruments. Skin Res Technol 10, 122-129 (2004).

207. Man, M.-Q. et al. Characterization of a hapten-induced, murine model with multiple features of atopic dermatitis: Structural, immunologic, and biochemical changes following single versus multiple oxazolone challenges. $J$ Invest Dermatol 128, 79-86 (2008). 
208. De Benedetto, A., Kubo, A. \& Beck, L.A. Skin barrier disruption: A requirement for allergen sensitization? J Invest Dermatol 132, 949-963 (2012).

209. Kim, D.-W., Park, J.-Y., Na, G.-Y., Lee, S.-J. \& Lee, W.-J. Correlation of clinical features and skin barrier function in adolescent and adult patients with atopic dermatitis. Int J Dermatol 45, 698-701 (2006).

210. Dearman, R.J. \& Kimber, I. Role of CD4(+) T helper 2-type cells in cutaneous inflammatory responses induced by fluorescein isothiocyanate. Immunology 101, 442-451 (2000).

211. Kaplan, D.H., Igyarto, B.Z. \& Gaspari, A.A. Early immune events in the induction of allergic contact dermatitis. Nat Rev Immunol 12, 114-124 (2012).

212. Imai, Y., Kondo, A., Iizuka, H., Maruyama, T. \& Kurohane, K. Effects of phthalate esters on the sensitization phase of contact hypersensitivity induced by fluorescein isothiocyanate. Clin Exp Allergy 36, 1462-1468 (2006).

213. Soumelis, V. et al. Human epithelial cells trigger dendritic cell mediated allergic inflammation by producing TSLP. Nat Immunol 3, 673-680 (2002).

214. Larson, R.P. et al. Dibutyl phthalate-induced thymic stromal lymphopoietin is required for Th2 contact hypersensitivity responses. J Immunol 184, 29742984 (2010).

215. Takeshita, K., Yamasaki, T., Akira, S., Gantner, F. \& Bacon, K.B. Essential role of MHC II-independent CD4+ T cells, IL-4 and STAT6 in contact hypersensitivity induced by fluorescein isothiocyanate in the mouse. Int Immunol 16, 685-695 (2004).

216. Moniaga, C.S. et al. Flaky tail mouse denotes human atopic dermatitis in the steady state and by topical application with Dermatophagoides pteronyssinus extract. Am J Pathol 176, 2385-2393 (2010).

217. Hewitson, J.P., Grainger, J.R. \& Maizels, R.M. Helminth immunoregulation: The role of parasite secreted proteins in modulating host immunity. Mol Biochem Parasitol 167, 1-11 (2009).

218. Oishi, S. et al. M2 polarization of murine peritoneal macrophages induces regulatory cytokine production and suppresses T-cell proliferation. Immunology 149, 320-328 (2016).

219. Ohl, L. et al. CCR7 governs skin dendritic cell migration under inflammatory and steady-state conditions. Immunity 21, 279-288 (2004).

220. Williams, H. et al. Is eczema really on the increase worldwide? J Allergy Clin Immunol 121, 947-954 e915 (2008). 
221. Masoli, M., Fabian, D., Holt, S., Beasley, R. \& Global Initiative for Asthma, P. The global burden of asthma: Executive summary of the GINA Dissemination Committee report. Allergy 59, 469-478 (2004). 